ORNLITM-1999/277

\title{
NEUTRON MONOCHROMATORS FOR RESIDUAL STRESS MAPPING AT THE NEW HB-2 BEAMPORT
}

\author{
A. D. Stoica ${ }^{*}$, M. Popovici, \\ Missouri University Research Reactor, Columbia, MO 65211, USA \\ C. R. Hubbard and S. Spooner \\ Oak Ridge National Laboratory, Oak Ridge, TN 37831-6064, USA \\ ${ }^{*}$ On leave from National Institute for Materials Physics, Bucharest, Romania
}

\section{BACKGROUND}

\subsection{Goals}

The main purpose of this work was to find the optimal monochromator configurations at the new HB-2 beamport for use in residual stress mapping. With that end in view it is essential to specify the following basic requirements.

1. Sampling volume is typically in the range of 0.5 to $10 \mathrm{~mm}^{3}$; a smaller volume is most desirable when achievable. The neutron flux falling on the sample rather than the full beam intensity is the relevant quantity to characterize the neutron beam.

2. Accuracy of stress measurements is influenced by the diffraction linewidth, the total number of counts under the measured peak, and the peak to background ratio.

3. The data collection time is determined by the accuracy level imposed on the final results and the number of measurements necessary to determine the orientation of the strain tensor. 
4. A large range of d-spacings must be accessible for residual strain measurements to cover the variety of materials and several diffraction peaks for each material desired by uscrs. The monochromator system has to include selectable wavelength options.

5. The monochromator system evaluation will include the impact of various shield and shutter designs. Cost and complexity of possible instrument modifications will be taken into account.

\subsection{Figure of merit}

To search for an optimal monochromator one needs to define an appropriate parameter to evaluate quality of the monochromator. A monochromator can be described by the phase space volume selected from the neutron source and the neutron transport efficiency from source to sample. The shape of the selected phase space volume is also important. The goal in strain measurements is to determine d-spacing changes very precisely. The figure of merit must be related to the accuracy in the peak position measurement.

The relative change of $d$-spacing is related to the peak shift by:

$$
\frac{\Delta d}{d}=\cot \theta_{s} \Delta \theta
$$

where $2 \theta_{s}$ is the detector (diffraction) angle. The standard deviation of $\Delta \mathrm{d} / \mathrm{d}$ is proportional to the standard deviation of the peak position $\left(\sigma_{2 \theta}\right)$ :

$$
\sigma_{\frac{\Delta d}{d}}=\frac{\cot \theta_{S}}{2} \sigma_{2 \theta}
$$

From statistical considerations, $\sigma_{2 \theta}$ is proportional to the variance $\sigma_{\mathrm{S}}$ of the diffraction line (line shape assumed to be Gaussian):

$$
\sigma_{2 \theta}=\frac{\sigma_{S}}{\sqrt{N}}
$$

where $\mathrm{N}$ is the integral number of counts. 
In the presence of background the accuracy will be worse than expression (1.3) prescribes. A Monte Carlo simulation has shown that the influence of a constant background can be quantified through a simple formula (see Fig. 1.1):

$$
\sigma_{2 \theta}=\frac{\sigma_{s}}{\sqrt{N}} \sqrt{1+3 \frac{F}{I}}
$$

where $F / I$ is the background to peak ratio. This ratio is a sample characteristic if the background comes from the sample and it also depends on the linewidth. In the following all estimates will be made under the assumption of zero background. To account for the influence of background the total number of counts should be divided by $(1+3 \mathrm{~F} / \mathrm{I})$.

The variance of the diffraction line contains an instrumental contribution $\sigma_{R}$ and a microstrain contribution $\sigma_{\mathrm{ms}}$ :

$$
\sigma_{S}^{2}=\sigma_{R}^{2}+4 \tan ^{2} \theta_{S} \sigma_{m s}^{2}
$$

The integral number of counts is proportional to the neutron flux at sample $\Phi_{S}$ (see section 1.1, first requirement) and for powder diffraction it is also proportional to:

$$
\frac{\lambda^{3}}{\sin \theta_{s} \sin 2 \theta_{s}}
$$

For a fixed d-spacing it results that:

$$
N \sim \tan \theta_{s} \Phi_{s} t
$$

where $t$ is the data collection time.

The figure of merit (the quality factor) will be in inverse proportion to the data collection time needed to reach a certain level of accuracy. Using expressions $(1.2,1.3$, $1.5,1.6)$ one can define the figure of merit as:

$$
F M=\frac{\cot \theta_{S} \Phi_{S}}{\sigma_{m s}^{2}+\frac{\cot ^{2} \theta_{S}}{4} \sigma_{R}^{2}}
$$

With this expression we will compare the accuracy of measurements made at different wavelengths. 


\subsection{Instrument design limitations}

The extraction of the beam dedicated to stress mapping measurements is sketched in Figs. 1.2 and 1.3. The beam is deflected $2^{\circ}$ from the HB- 2 beam line axis. At higher deflection angles the neutron beam would hit structural parts of the reactor wall. The monochromator locates at $768-788 \mathrm{~cm}$ from the end of the neutron beam pipe near the reactor core. A natural beam divergence of about $1^{\circ}$ in horizontal and vertical planes results from this geometry. A first task was to size the opening in the primary beam shutter.

The secondary beam diffracted from the monochromator will penetrate the shielding through an experiment shutter. The basic configuration of the stress mapping diffractometer is: sketched in Fig. 1.4. The minimum distance between the monochromator and the goniometer center is $212 \mathrm{~cm}$. The current experiment shutter design prescribes an opening of $7.6 \mathrm{~cm}$ wide and $12.7 \mathrm{~cm}$ high. A second task was to determine if this opening is adequate.

The Monte Carlo method was used to calculate the reduction of neutron intensity at the monochromator due to the size of the primary beam shutter. The maximum monochromator dimensions were considered $(16.5 \mathrm{~cm}$ wide $\times 15.2 \mathrm{~cm}$ high - the clearance gauge of the existing monochromator unit). The transmission was calculated relative to the total number of neutrons striking the monochromator area where there were no limitations between the monochromator and the neutron source (circular disk with $18 \mathrm{~cm}$ diameter). Results are given in Table 1.1.

At a square opening of $9-10 \mathrm{~cm}$ the shutter transmission is $60-70 \%$. This evaluation is not complete because focusing monochromators cannot reflect all neutron trajectories between the neutron source and monochromator.

To evaluate the monochromator arrangement a fixed take-off angle $\left(88^{\circ}\right)$ and a fixed distance from monochromator to sample were considered. The sample was assumed to be point-like. Trajectories arriving at the point sample from an infinitely extended neutron source were generated by the Monte Carlo method. The extraction efficiency was defined as the ratio of the number of neutrons actually passing through the system (finite source, shutters and monochromator) to the total number of reflected 
neutrons coming from an indefinitely large source. This efficiency depends on (1) the monochromator curvature radii in the horizontal $\left(\mathrm{R}_{\mathrm{H}}\right)$ and vertical $\left(\mathrm{R}_{\mathrm{V}}\right)$ planes, (2) the cutting angle $\left(\chi_{M}\right.$, between the crystallographic plane that reflects neutrons and the monochromator surface) and, (3) the openings of both the primary beam and experiment shutters.

Table 1.1

\begin{tabular}{|c|c|c|c|c|c|c|}
\hline $\begin{array}{c}\text { Vertical } \\
\text { opening }\end{array}$ & \multicolumn{7}{|c|}{$\begin{array}{c}\text { Primary beam shutter horizontal opening } \\
\text { [cm] }\end{array}$} \\
\hline [cm] & 7 & 8 & 9 & 10 & 11 & 12 \\
\hline 7 & 0.39 & 0.45 & 0.49 & 0.54 & 0.58 & 0.60 \\
\hline 8 & 0.45 & 0.51 & 0.56 & 0.60 & 0.65 & 0.67 \\
\hline 9 & 0.49 & 0.55 & 0.62 & 0.66 & 0.71 & 0.74 \\
\hline 10 & 0.52 & 0.59 & 0.65 & 0.71 & 0.76 & 0.79 \\
\hline 11 & 0.55 & 0.63 & 0.69 & 0.74 & 0.79 & 0.84 \\
\hline 12 & 0.58 & 0.66 & 0.72 & 0.78 & 0.83 & 0.87 \\
\hline
\end{tabular}

The extraction efficiency for the case of no limitations except for the finite size of the source is presented in Fig. 1.5 as a function of the cutting angle and the horizontal radius of curvature, at fixed value of vertical radius $\left(R_{V}=1.75 \mathrm{~m}\right)$. A large strip with high extraction efficiency is visible. The effect of introducing the shutter opening is shown in Fig. 1.6. The results are shown for the primary beam shutter with an opening of $10 \times 10 \mathrm{~cm}$ and for the experiment shutter with the maximum available opening of $7.6 \times 12.7 \mathrm{~cm}$. The extraction efficiency is visibly reduced for reflection asymmetries with beam extension $\left(\chi_{M}<0\right)$. To show this effect more clearly, the variation of the extraction efficiency with cutting angle is shown in Fig. 1.7, with the horizontal radius set at optimal value. The limitation by the second shutter is responsible for the reduction of the extraction efficiency below $\chi_{M}=15^{\circ}$. To show the effect of the first shutter, the same dependence is presented in Fig. 1.8 for a relaxed 
opening of the second shutter. It is seen that an extraction efficiency exceeding $80 \%$ can be reached with a horizontal opening of $10 \mathrm{~cm}$ at most cutting angles $\left(\chi_{M}<35^{\circ}\right)$.

The effect of the vertical curvature is shown in Fig. 1.9. The horizontal radius was fixed at the optimum value and the opening of the second shutter was relaxed. The largest efficiency of extraction is reached at radii of vertical curvature exceeding 1.8 $\mathrm{m}$. The vertical opening of the first shutter $(10 \mathrm{~cm})$ reduces the extraction efficiency at vertical radii exceeding $2.2 \mathrm{~m}$. At $\mathrm{Rv}_{\mathrm{v}}=1.8 \mathrm{~m}$ the vertical opening of the second shutter can be reduced to $5 \mathrm{~cm}$ with no significant loss of extraction efficiency. However, an excessive reduction of the height of the shutter is not recommended. Our analysis considered the case of focusing neutrons at $2.12 \mathrm{~m}$ from the monochromator, but other configurations, like a double crystal arrangement, should not be excluded.

In conclusion, an opening size of $10 \times 10 \mathrm{~cm}$ is recommended for the primary beam shutter, although a $9 \times 9 \mathrm{~cm}$ size is also acceptable. The maximum allowed opening size of the experiment shutter $(7.6 \times 12.7 \mathrm{~cm})$ would reduce the extraction efficiency by as much as $50 \%$. A monochromator cutting angle above $15^{\circ}$ is recommended to minimize this loss.

In the following chapters two different monochromator configurations are discussed: single reflection and double reflection. For single reflection (section. 2) the performance of two types of focusing monochromators, made of thick silicon plates and of packets of thin wafers, are compared. The best choice for neutron stress mapping permitted by the new HB-2 geometry is searched for. In section 3 the double reflection option is analyzed. The search focuses on two basic arrangements: with identical crystals at $\sim 55^{\circ}$ take-off angle and with non-identical crystals at $\sim 88^{\circ}$ takeoff angle for the first crystal (to preserve the single reflection option at the same takeoff angle). The final recommendations are given in section 4 . 


\section{SINGLE REFLECTION MONOCHROMATOR UNITS}

\subsection{Focusing monochromators for diffraction}

Mosaic crystal monochromators are the basic choice for conventional neutron diffractometry with Soller collimators. Highly oriented pyrolytic graphite (PG) and mosaic Be give the highest beam intensities, but the d-spacing of PG is too large for adequate resolution in diffraction while good $\mathrm{Be}$ crystals are not readily available. $\mathrm{Zn}$, $\mathrm{Cu}$ and $\mathrm{Ge}$ mosaic crystals are commonly used [1]. In conventional configurations good resolution in diffraction is obtained with tight collimation at large monochromator take-off angles [2]. Much work has been done to focus the neutron beam in the vertical plane. The most successful version of mosaic crystal monochromator for high resolution diffraction is a stack of flat plates of squashed $\mathrm{Ge}$, each tilted vertically to provide vertical beam concentration onto the sample [3]. The mosaic spread sets a limit to spatial focusing: a spread of $20^{\prime}$ gives a blurring of about $2 \mathrm{~cm}$ for each meter of distance.

Alternative monochromators based on elastically deformed perfect crystals have been developed in recent years [4]. They allow focusing in both the horizontal and vertical planes and a control of resolution through focusing in scattering. In spite of lower full beam intensities, under focusing conditions bent perfect crystals can deliver neutrons to small areas at a higher flux than mosaic crystals can. This is because bent perfect crystals have high peak reflectivity [5] and can accept beams of large angular divergence. The best choice for a diffractometer with small gauge volume and position sensitive detector (PSD) is a double focusing bent perfect crystal monochromator [6].

A curved crystal acts as a neutron lens [7]. A thin bent monochromator placed at a distance $L_{0}$ from a neutron source will produce an image of the source at a distance $L_{1}$ from the crystal, related to $L_{0}$ by the simple lens formula:

$$
\mathrm{f}_{\mathrm{H}} / \mathrm{L}_{\mathrm{O}}+\mathrm{f}_{\mathrm{H}}{ }^{*} / \mathrm{L}_{1}=1 \quad \text { (in the horizontal plane). }
$$


The main difference between a bent crystal and a lens is that the focal lengths before reflection $\left(\mathrm{f}_{\mathrm{H}}\right)$ and after reflection $\left(\mathrm{f}_{\mathrm{H}}{ }^{*}\right)$ are different:

$$
\begin{aligned}
& f_{H}=\left(R_{H} / 2\right) \sin \left(\theta_{M}+\chi_{M}\right) \\
& f_{H}{ }^{*}=\left(R_{H} / 2\right) \sin \left(\theta_{M}-\chi_{M}\right)
\end{aligned}
$$

The following notations were used: $\theta_{M}$ the Bragg angle, $\chi_{M}$ the cutting angle (the angle between the lattice plane and the crystal surface) and $R_{H}$ the crystal radius of curvature in the plane of reflection (horizontal, or equatorial).

The distance $L_{1}$ ' to the vertical plane image is given by the same relation with other focal lengths:

$$
\begin{aligned}
& f_{V} / L_{0}+f_{V} / L_{1}{ }^{\prime}=1, \\
& f_{V}=R_{V} /\left(2 \cos \chi_{M} \sin \theta_{M}\right)
\end{aligned}
$$

where $f_{V}$ is the vertical focal length, the same before and after reflection, and $R_{V}$ is the sagittal (vertical) radius of curvature. The 'astigmatic difference' of $f_{V}$ in relation to $f_{H}$ and $\mathrm{f}_{\mathrm{H}}{ }^{*}$ is a significant distinction between neutron Bragg optics and geometrical optics.

The Bragg reflection by a bent crystal introduces a correlation in the wavevector space between the modulus of the wavevector $\mathbf{k}$ and the neutron direction in the horizontal plane [8]. This correlation can be manipulated to achieve a suitable orientation of the instrument resolution ellipsoid [9]. Focusing in powder diffraction for a sample of small diameter occurs when [9]:

$$
\mathrm{f}_{\mathrm{H}}{ }^{*} / \mathrm{L}_{1}=1 /\left(2+\tan \theta_{\mathrm{M}} / \tan \theta_{\mathrm{S}}\right)
$$

where $L_{1}$ is the distance between the monochromator and the sample and $2 \theta_{\mathrm{S}}$ is the detector (diffraction) angle. This relation defines the radius of curvature needed to achieve good resolution in powder diffraction at a given detector angle. The numerical values of the resolution and intensity are determined by the monochromator thickness, the elastic constants of the crystal material, and the type of bending. Relation (2.5) gives a neutron equivalent of the focusing at detector in X-ray powder diffraction (the Bragg-Brentano parafocusing). Characteristic monochromatic X-rays come from a point source, are diffracted by an extended plate sample, and go to the same point of detection. Neutrons, with no point sources and no strictly monochromatic beams, 
come from an extended crystal, go to a point sample and - under focusing conditions go to the same point of detection.

Silicon is the commercially available material for focusing monochromators with high transparency for neutrons. Early focusing neutron monochromators were bent pneumatically (quasi-spherically) [10] or mechanically (cylindrically) [11]. The latest focusing monochromators of high performance have a more complex design that will be described in the following two sections.

\subsection{Bulk silicon crystal unit}

One unit developed at the Missouri University Research Reactor (MURR) [4] is made of 9 vertically stacked $\mathrm{Si}$ blades, mechanically bent in the horizontal (diffraction) plane and quasi-bent by segmentation in the vertical plane. All blades originate from the same plate, which automatically ensures their correct relative orientation. The plate is cut obliquely from an 8 " (203.2 mm) diameter [100] ingot with a suitable cutting angle and, usually, with the $\langle 011\rangle$ zone axis vertical.

The horizontal radius $R_{H}$ is given by a 4-point elastic bending device and is adjustable with a fine screw. A stepping motor can be mounted to remotely drive the adjusting screw. The vertical curvature $R_{V}$ is set by the profile of the bending posts (barrel shaped on the front, concave on the back) and is fixed. A difference from the usual 4-point bending method is that the mobile posts revolve around axes that are close to the center of the device. This eliminates the nonuniformity of the horizontal curvature over the height of the stack due to the varying thickness of the posts.

The usable monochromator area is $16.5 \mathrm{~mm}$ wide and $15.2 \mathrm{~mm}$ high. The blade thickness is usually between 5 and $6 \mathrm{~mm}$. The horizontal curvature is limited by the breaking limit (safe when $R_{H}>10 \mathrm{~m}$ ) of the silicon blades. Because the second focal length $\mathrm{f}_{\mathrm{H}}{ }^{*}$ must be comparable to the distance between monochromator and sample, which usually is short (1-2 m), the asymmetric reflection with beam condensation (Fankuchen range) is used. The cutting angle is close to the Bragg angle so that the radius of curvature is considerably larger than the second focal distance (see relation $2.2 b)$, thus avoiding breakage. 
The neutron reflectivity of bent perfect crystals is well described by the - lamellar approximation [5]. The local value of the reciprocal lattice vector is defined for every point of the crystal by taking into account the variation, due to the elastic deformation, of the orientation and spacing of lattice planes. It is assumed that all neutrons along a given path in the crystal are reflected with a constant probability when the Bragg condition is fulfilled.

By neglecting the intrinsic dynamic diffraction range (Darwin width, of a few seconds of arc) the diffraction by an elastically bent perfect crystal becomes deterministic. The point where the reflection occurs is determined uniquely by the direction and the wave vector of the incoming neutron. The path of the reflected neutron is also determined uniquely. The crystal dimensions will thus fully define the domain in real and phase space where the Bragg reflection is possible.

A matrix method similar to that used in Gaussian lens optics has been worked out for neutron optics $[8,10]$. The neutron state before reflection is specified by the spatial coordinates across the beam $\left(y_{i}, z_{i}\right)$, the angular deviations $\left(\gamma_{i}, \delta_{i}\right)$ from the beam axis and the relative deviation of the wavevector $(\Delta \mathrm{k} / \mathrm{k})$. The neutron state is actually defined in the five dimensions by the phase space vector $\left(y_{i}, k \gamma_{i}, \Delta k, z_{i}, k \delta_{i}\right)$. The neutron state after reflection is specified in the same way by changing the subscript $(i \rightarrow f)$. In the paraxial approximation of linear relations describing the Bragg reflection the neutron coordinates in the horizontal (diffraction) plane are not correlated with those in the vertical plane and the wave vector deviation has no influence on the vertical motion. One can thus consider separately the vectors $v_{i}=\left(y_{i}\right.$, $\left.\gamma_{i}, \Delta k / k\right)$ and $v_{f}=\left(y_{f}, \gamma_{f}, \Delta k / k\right)$ of the neutron state in the horizontal plane and the vectors $\mathbf{u}_{\mathrm{i}}=\left(\mathrm{z}_{\mathrm{i}}, \delta_{\mathrm{i}}\right)$ and $\mathbf{u}_{\mathrm{f}}=\left(\mathrm{z}_{\mathrm{f}}, \delta_{\mathrm{f}}\right)$ in the vertical plane.

The vectors $\mathbf{v}_{\mathrm{i}}$ and $\mathbf{v}_{\mathrm{f}}$ are related by the Bragg reflection matrix $S_{\mathbf{H}}$ :

$$
\mathbf{v}_{\mathbf{f}}=\mathbf{S}_{\mathbf{H}} * \mathbf{v}_{\mathbf{i}}
$$

with the following elements:

$$
\begin{aligned}
& S_{\mathrm{H} 11}=\varepsilon_{\mathrm{i}} ; \mathrm{S}_{\mathrm{H} 12}=-\varepsilon \mathrm{R}_{\mathrm{H}} ; \mathrm{S}_{\mathrm{H} 13}=\varepsilon \mathrm{R}_{\mathrm{H}} \tan \theta ; \\
& \mathrm{S}_{\mathrm{H} 21}=-\left(\varepsilon_{\mathrm{i}} \varepsilon_{\mathrm{f}}-1\right) /\left(\varepsilon R_{\mathrm{H}}\right) ; \mathrm{S}_{\mathrm{H} 22}=\varepsilon_{\mathrm{f}} ; \mathrm{S}_{\mathrm{H} 23}=-\left(\varepsilon_{\mathrm{f}}+1\right) \tan \theta ; \\
& \mathrm{S}_{\mathrm{H} 31}=\mathrm{S}_{\mathrm{H} 32}=0 ; \mathrm{S}_{\mathrm{H} 33}=1 .
\end{aligned}
$$

The following notations were used: 


$$
\begin{aligned}
& \Delta \mathrm{k} / \mathrm{k}-\text { relative wavevector deviation; } \\
& \mathrm{G}=\left(\cos \chi_{\mathrm{M}} / \cos \theta_{M}\right)\left[1-(1+\kappa) \sin \left(\theta_{M}+\chi_{M}\right) \sin \left(\theta_{M}-\chi_{M}\right)\right] \\
& \kappa-\text { material elasticity constant; } \\
& \varepsilon=\sin 2 \theta_{M} / G ; \\
& \varepsilon_{i}=\left[\varepsilon-\sin \left(\theta_{M}-\chi_{M}\right)\right] / \sin \left(\theta_{M}+\chi_{M}\right) ; \\
& \varepsilon_{f}=\left[\varepsilon-\sin \left(\theta_{M}+\chi_{M}\right)\right] / \sin \left(\theta_{M}-\chi_{M}\right) .
\end{aligned}
$$

Physically $G / R_{H}$ represents the change in Bragg angle per unit incident neutron pathlength in the crystal [5]. The material elasticity constant $\kappa$ is the ratio between the deformation tensor components along and normal to the crystal surface. It depends on the crystallographic orientation of the crystal plate and on the method of bending, and it equals the Poisson ratio in a particular case [5].

The matrix $\mathbf{S}_{\mathbf{H}}$ is unitary (its determinant equals unity). This means that the phase space volume is preserved upon the transformation (2.6), as required by the Liouville theorem (which actually says that the neutron density in the phase space cannot increase). The elements of the inverse matrix $\mathbf{S}_{\mathbf{H}}{ }^{-1}$ are:

$$
\begin{aligned}
& S_{\mathrm{H} 11}^{\prime}=\varepsilon_{\mathrm{f}} ; \mathrm{S}_{\mathrm{H} 12}^{\prime}=\varepsilon R_{\mathrm{H}} ; \mathrm{S}_{\mathrm{H} 13}^{\prime}=\varepsilon R_{\mathrm{H}} \tan \theta_{\mathrm{M}} ; \\
& S_{\mathrm{H} 21}^{\prime}=\left(\varepsilon_{\mathrm{i}} \varepsilon_{\mathrm{f}}-1\right) /\left(\varepsilon R_{\mathrm{H}}\right) ; S_{\mathrm{H} 22}^{\prime}=\varepsilon_{\mathrm{i}} ; \mathrm{S}_{\mathrm{H} 23}^{\prime}=\left(\varepsilon_{\mathrm{i}}+1\right) \tan \theta_{\mathrm{M}} ; \\
& S_{\mathrm{H} 31}^{\prime}=S_{\mathrm{H}^{\prime} 32}=0 ; S_{\mathrm{H} 33}^{\prime}=1 .
\end{aligned}
$$

Bragg reflection can take place only if the following relation is fulfilled:

$$
\left|\mathbf{A} \mathbf{v}_{\mathrm{i}}\right|<\mathbf{B}
$$

where the components of $\mathbf{A}$ and $\mathbf{B}$ are:

$$
\begin{aligned}
& A_{11}=-1 / G ; A_{12}=\left[\sin \left(\theta_{M}+\chi_{M}\right) / G\right] R_{H} ; A_{13}=-\left[\sin \left(\theta_{M}+\chi_{M}\right) / G\right] R_{H} \tan \theta_{M} ; \\
& A_{21}=-\cot \left(\theta_{M}+\chi_{M}\right) / G+1 / \sin \left(\theta_{M}+\chi_{M}\right) ; A_{22}=\left[\cos \left(\theta_{M}+\chi_{M}\right) / G\right] R_{H} ; \\
& A_{23}=-\left[\cos \left(\theta_{M}+\chi_{M}\right) / G\right] R_{H} \tan \theta_{M} ; \\
& B_{1}=g / 2 ; B_{2}=l / 2,
\end{aligned}
$$

with $g$ the crystal thickness and $l$ the crystal length;

A similar relation holds for the vector $\mathbf{v}_{\mathbf{f}}$ with the following coefficients:

$$
\begin{aligned}
& \mathrm{A}^{\prime}{ }_{12}=-1 / \mathrm{G} ; \mathrm{A}^{\prime}{ }_{12}=-\left[\sin \left(\theta_{\mathrm{M}}-\chi_{\mathrm{M}}\right) / \mathrm{G}\right] \mathrm{R}_{\mathrm{H}} ; \mathrm{A}^{\prime}{ }_{13}=-\left[\sin \left(\theta_{\mathrm{M}}-\chi_{\mathrm{M}}\right) / \mathrm{G}\right] \mathrm{R}_{\mathrm{H}} \tan \theta_{\mathrm{M}} ; \\
& A^{\prime}{ }_{21}=\cot \left(\theta_{M}-\chi_{M}\right) / G-1 / \sin \left(\theta_{M}-\chi_{M}\right) ; A^{\prime}{ }_{22}=\left[\cos \left(\theta_{M}-\chi_{M}\right) / G\right] R_{H} ; \\
& A^{\prime}{ }_{23}=\left[\cos \left(\theta_{M}-\chi_{M}\right) / G\right] R_{H} \tan \theta_{M}
\end{aligned}
$$


The vectors $\mathbf{u}_{\mathrm{i}}=\left(\mathrm{z}_{\mathrm{i}}, \delta_{\mathrm{i}}\right)$ and $\mathbf{u}_{\mathrm{f}}=\left(\mathrm{z}_{\mathrm{f}}, \delta_{\mathrm{f}}\right)$ are related by the vertical matrix $\mathbf{S}_{\mathbf{v}}$ :

$$
\mathbf{u}_{\mathrm{f}}=\mathbf{S}_{\mathbf{v}} \mathbf{u}_{\mathrm{i}}
$$

with the following elements:

$$
\begin{aligned}
& \mathrm{S}_{\mathrm{V} 11}=1 ; \mathrm{S}_{\mathrm{V} 12}=0 ; \\
& \mathrm{S}_{\mathrm{V} 21}=-2 \sin \theta_{\mathrm{M}} \cos \chi_{\mathrm{M}} / \mathrm{R}_{\mathrm{V}} ; \mathrm{S}_{\mathrm{V} 22}=1 .
\end{aligned}
$$

A simple restriction holds: $\left|\mathrm{z}_{\mathrm{i}}\right|=\left|\mathrm{z}_{\mathrm{f}}\right|<h / 2$, with $h$ the crystal height.

The peak reflectivity is given by the formula:

$$
\mathrm{P}=1-\exp \left(-\mathrm{Q}\left|\mathrm{R}_{\mathrm{H}} / \mathrm{G}\right|\right) \text {. }
$$

where $\mathrm{Q}=\left(\Delta \mathrm{k}_{0} / \mathrm{D}\right) \tan \theta_{\mathrm{M}} / \mathrm{k}=\left(\Delta \mathrm{k}_{0} / \mathrm{D}\right)(\mathrm{d} / \pi)\left(\sin ^{2} \theta_{\mathrm{M}} / \cos \theta_{\mathrm{M}}\right)$ is the kinematic reflectivity per unit incident neutron pathlength in the crystal, $d$ is the lattice spacing for the given reflection and $\Delta \mathrm{k}_{0} / \mathrm{D}$ is the Maier-Leibnitz reflectivity constant (selected wavevector band per unit path) [12]. The values of $d$ and $\Delta k_{0} / D$ for silicon are given in Table 2.1.

Table 2.1

\begin{tabular}{|c|c|c|}
\hline Reflection & $\mathrm{d}[\mathrm{A}]$ & $\Delta \mathrm{k}_{0} / \mathrm{D}\left[\mathrm{A}^{-1} \mathrm{~cm}^{-1}\right]$ \\
\hline$(111)$ & 3.1355 & 0.0273 \\
\hline$(220)$ & 1.9200 & 0.0200 \\
\hline$(311)$ & 1.6374 & 0.0068 \\
\hline$(400)$ & 1.3577 & 0.0098 \\
\hline$(331)$ & 1.2459 & 0.0038 \\
\hline$(422)$ & 1.1086 & 0.0064 \\
\hline$(511)$ & 1.0451 & 0.0026 \\
\hline
\end{tabular}

The relations presented above form the basis of a matrix neutron code for design and optimization of diffraction arrangements. Results of computations with the code have been presented in a series of papers $[9,10]$ and also in a previous project report to ORNL. A later development in phase space diagram computation has occurred in part under the present project. A code has been worked out for arrangements of single or multiple monochromators. It makes use of the matrices $\mathbf{S}_{\mathbf{H}}$, 
$\mathbf{S}_{\mathbf{v}}$ defined above and also of translation matrices. Translation matrices describe the motion of neutrons in empty space between two spectrometer elements. The space phase diagrams visualize the boundaries of the allowcd phase space domain. The diagrams are computed from the geometry of the experiment, with all limitations due to the presence of various slits accounted for.

\subsection{Multi-wafer silicon unit}

Silicon breakage restricts the range of applications of bent bulk silicon monochromators. A way to overcome the restriction is to use monochromators made of packets of thin silicon wafers. Such monochromators allow for a smaller bending radius, hence for a stronger spatial focusing, and give more flexibility in the design of focusing arrangements.

A prototype was made of a packet of 14 commercial wafers, each of $20 \mathrm{~cm}$ diameter and $0.7 \mathrm{~mm}$ thickness, originating from the same $\mathrm{Si}[100]$ ingot. The bending device was basically the same as for bulk silicon crystals. The overall crystal thickness was about $10 \mathrm{~mm}$, but designs having thickness between $3 \mathrm{~mm}$ and $15 \mathrm{~mm}$ can be fabricated. The vertical curvature is obtained by fine segmentation $(5 \mathrm{~mm})$. The unit has an adjustable horizontal curvature with a minimum radius of about $1 \mathrm{~m}$ (the breaking limit for commercial wafers). In the vertical plane the radius is fixed by the profile of bending posts.

Modeling of multi-wafer units has required the introduction of a new option in the computer design codes. Work on the necessary modifications has been done partially under the present project. Some of the results are presented below.

Each wafer in the packet reflects neutrons like a bulk crystal, but problems arise upon considering the reflectivity of the packet as a whole. On the $\theta$ scale (or $\Delta \mathrm{k} / \mathrm{k}$ scale) the reflectivity of a packet of wafers is generally comb-shaped. The width of a tooth (the band reflected by each wafer) is defined by the value of the elastic stress, but the distance between successive teeth is given by the geometry of bending only. The filling ratio is thus of concern. The reflectivity curve is approximated by a rectangle having the same overall width and a height equal to the average reflectivity 
over the many teeth. The filling ratio must be considered not solely on the $\theta$ or $\Delta \mathrm{k} / \mathrm{k}$ scales, but in the three-dimensional phase space. If the reflection is asymmetric, the overall phase space volumes before and after reflection differ, leading to different phase space filling ratios. When the teeth superimpose, corrections must be introduced in the effective peak reflectivity to account for possible losses due to multiple reflections.

In ray tracing calculations, to simplify the calculations, the packet of wafers was simulated by a bulk bent crystal without elastic stresses. The elements of the Bragg reflection matrix $\mathbf{S}_{\mathbf{H}}$ are:

$$
\begin{aligned}
& \mathrm{S}_{\mathrm{H} 11}=\cos \left(\theta_{\mathrm{M}}-\chi_{\mathrm{M}}\right) / \cos \left(\theta_{\mathrm{M}}+\chi_{\mathrm{M}}\right) ; \mathrm{S}_{\mathrm{H} 12}=-2 \mathrm{~L}_{2}^{*} ; \mathrm{S}_{\mathrm{H} 13}=2 \mathrm{~L}_{2}^{*} \tan \theta_{\mathrm{M}} ; \\
& \mathrm{SH}_{21}=0 ; \mathrm{SH}_{22}=1 ; \mathrm{SH}_{23}=-2 \tan \theta_{\mathrm{M}} ; \\
& \mathrm{SH}_{31}=\mathrm{SH}_{32}=0 ; \mathrm{SH}_{33}=1 .
\end{aligned}
$$

The determinant of $\mathbf{S}_{\mathbf{H}}$ is $\cos \left(\theta_{\mathrm{M}}-\chi_{\mathrm{M}}\right) / \cos \left(\theta_{\mathrm{M}}+\chi_{\mathrm{M}}\right)$, and it is different from unity if the reflection is asymmetric. The overall volumes in the phase space may thus be different before and after reflection. This is an apparent violation of the Liouville theorem, introduced by the approximation used in modeling. We know that no violation occurs in reality as each individual wafer obeys the Liouville theorem. To correct for this situation, a factor is introduced in the average peak reflectivity, which comes to depend on whether the phase space volume considered on its averaging is before or after reflection.

The elements of the inverse matrix $\mathbf{S}_{\mathbf{H}}{ }^{-1}$ are:

$\mathrm{S}_{\mathrm{H} 11}{ }_{11}=\cos \left(\theta_{\mathrm{M}}+\chi_{\mathrm{M}}\right) / \cos \left(\theta_{\mathrm{M}}-\chi_{\mathrm{M}}\right) ; \mathrm{S}_{\mathrm{H} 12}{ }_{12}=2 \mathrm{~L}_{1}^{*} ; \mathrm{S}_{\mathrm{H} 13}{ }_{13}=2 \mathrm{~L}_{1}^{*} \tan \theta_{\mathrm{M}} ;$

$\mathrm{S}_{\mathrm{H} 21}=0 ; \mathrm{S}_{\mathrm{H} 22}^{\prime}=1 ; S_{\mathrm{H} 23}^{\prime}=2 \tan \theta_{\mathrm{M}}$;

$\mathrm{S}_{\mathrm{H} 31}{ }^{\prime}=\mathrm{S}_{\mathrm{H} 32}{ }^{\prime}=0 ; \mathrm{S}_{\mathrm{H} 33}{ }^{\prime}=1$.

where $L_{1}^{*}=R_{H} \sin 2 \theta_{M} / 2 \cos \left(\theta_{M}-\chi_{M}\right)$ and $L_{2}^{*}=R_{H} \sin 2 \theta_{M} / 2 \cos \left(\theta_{M}+\chi_{M}\right)$ are characteristic lengths that equal each other for symmetric reflection.

The reflection can take place only if the following relation is fulfilled:

$$
\left|\mathbf{A} \mathbf{v}_{1}\right|<\mathbf{B}
$$

where the components of $\mathbf{A}$ and $\mathbf{B}$ are:

$$
\begin{aligned}
& A_{11}=-1 / \cos \left(\theta_{M}+\chi_{M}\right) ; A_{12}=R_{H} \tan \left(\theta_{M}+\chi_{M}\right) ; A_{13}=-R_{H} \tan \left(\theta_{M}+\chi_{M}\right) \tan \theta_{M} ; \\
& A_{21}=0 ; A_{22}=R_{H} ; A_{23}=-R_{H} \tan \theta_{M} ;
\end{aligned}
$$




$$
\mathrm{B}_{1}=g / 2 ; \mathrm{B}_{2}=l / 2 .
$$

The relation (2.10) defines the boundaries of the phase space acceptance window (before reflection by the bent crystal).

A similar relation $\left|\mathbf{A}^{\prime} \mathbf{v}_{\mathbf{f}}\right|<\mathbf{B}$ holds for the vector $\mathbf{v}_{\mathbf{f}}$ with the following coefficients:

$$
\begin{aligned}
& \mathrm{A}^{\prime}{ }_{12}=-1 / \cos \left(\theta_{\mathrm{M}}-\chi_{\mathrm{M}}\right) ; \mathrm{A}_{12}^{\prime}=-\mathrm{R}_{\mathrm{H}} \tan \left(\theta_{\mathrm{M}}-\chi_{\mathrm{M}}\right) ; \mathrm{A}^{\prime}{ }_{13}=-\mathrm{R}_{\mathrm{H}} \tan \left(\theta_{\mathrm{M}}-\chi_{\mathrm{M}}\right) \tan \theta_{\mathrm{M}} ; \\
& \mathrm{A}^{\prime}{ }_{21}=0 ; \mathrm{A}^{\prime}{ }_{22}=\mathrm{R}_{\mathrm{H}} ; \mathrm{A}^{\prime}{ }_{23}=\mathrm{R}_{\mathrm{H}} \tan \theta_{\mathrm{M}} .
\end{aligned}
$$

The above relations define the phase space emergence window (after reflection).

The relations between $\mathbf{u}_{\mathbf{f}}$ and $\mathbf{u}_{\mathbf{i}}$ and the vertical restriction are the same as for the bulk bent crystal (2.8).

To compensate for the difference in the volumes of the acceptance and emergence phase space windows, the peak reflectivity is multiplied by $\cos \left(\theta_{M}+\chi_{M}\right) / \cos \left(\theta_{M}-\chi_{M}\right)$, that is the peak reflectivity before $\left(P_{a}\right)$ and after $\left(P_{b}\right)$ reflection are related by:

$$
P_{a}=\left|\cos \left(\theta_{M}+\chi_{M}\right) / \cos \left(\theta_{M}-\chi_{M}\right)\right| P_{b}
$$

The correction of the reflectivity for the phase space filling ratio is introduced by the formulae:

$$
\begin{aligned}
& P_{b}=\left|G / \cos \left(\theta_{M}+\chi_{M}\right)\right| P_{0} \text { when }\left|G / \cos \left(\theta_{M}+\chi_{M}\right)\right|<1, \\
& P_{a}=\left|G / \cos \left(\theta_{M}-\chi_{M}\right)\right| P_{o} \text { when }\left|G / \cos \left(\theta_{M}-\chi_{M}\right)\right|<1,
\end{aligned}
$$

with $\mathrm{P}_{0}=1-\exp \left(-\mathrm{Q} \mid \mathrm{R}_{\mathrm{H}} / \mathrm{Gl}\right)$ as given by the bulk case formula (2.9).

If $\left|G / \cos \left(\theta_{M}+\chi_{M}\right)\right|>1$ the wafers screen each other in the incident beam. An extinction factor has to be introduced to account for this screening. The expression of the factor will depend on the superposition number $n=I n t\left|G / \cos \left(\theta_{M}+\chi_{M}\right)\right|$ and on the number of wafers in the packet $\mathrm{N}$. At high $\mathrm{N}$ the following asymptotic expression may be used:

$$
P_{b, a s}=\left[n+1-\left|G / \cos \left(\theta_{M}+\chi_{M}\right)\right|\right]\left[1-\left(1-P_{0}\right)^{n}\right]+\left[\left|G / \cos \left(\theta_{M}+\chi_{M}\right)\right|-n\right]\left[1-\left(1-P_{0}\right)^{n+1}\right]
$$

For a finite number of wafers the final formula at $N>n$ is:

$$
\mathrm{P}_{b}=\frac{\mathrm{N}-n}{\mathrm{~N}} \mathrm{P}_{b, a s}+\frac{1}{\mathrm{~N}} \sum_{j=0}^{n-1}\left[\mathrm{G} / \cos \left(\theta_{M}+\chi_{M}\right)+\mathrm{n}-2 \mathrm{j}-1\right] \mathrm{P}_{0}\left(1-\mathrm{P}_{0}\right)^{j}
$$

and if $\mathrm{N}<\mathrm{n}$ one obtains: 


$$
\mathrm{P}_{b}=\frac{1}{\mathrm{~N}} \sum_{j=0}^{N-1}\left[\mathrm{G} / \cos \left(\theta_{M}+\chi_{M}\right)+\mathrm{n}-2 \mathrm{j}-1\right] \mathrm{P}_{0}\left(1-\mathrm{P}_{0}\right)^{j}
$$

The formula (2.13) was derived under the assumption of no superposition and no multiple scattering in the reflected beam. Superposition in the reflected beam exists when the superposition number $m=\operatorname{Int}\left|G / \cos \left(\theta_{M}-\chi_{M}\right)\right|$ is different from zero. The wafers will then screen each other after reflection, causing the neutrons to be scattered out of the beam by multiple reflection. The expression (2.13) must be multiplied by a factor accounting for multiple reflection $\left(\mathrm{f}_{\text {mix }}\right)$ :

$$
f_{\text {mix }}=\left(1-P_{-}\right) /\left(1-P_{+} P_{-}\right)
$$

where $P_{.}$and $P_{+}$are different from 0 when $m$ and $n$ (respectively) are different from 0 . The corresponding expressions for $\mathrm{P}_{-}$and $\mathrm{P}_{+}$can be inferred in a similar way to (2.13), with only a change in exponents:

$$
\begin{aligned}
& P_{.}=\left[m+1-\left|G / \cos \left(\theta_{M}-\chi_{M}\right)\right|\right]\left[1-\left(1-P_{0}\right)^{m-1}\right]+\left[\left|G / \cos \left(\theta_{M}-\chi_{M}\right)\right|-m\right]\left[1-\left(1-P_{0}\right)^{m}\right] \\
& P_{+}=\left[n+1-\left|G / \cos \left(\theta_{M}+\chi_{M}\right)\right|\right]\left[1-\left(1-P_{0}\right)^{n-1}\right]+\left[\left|G / \cos \left(\theta_{M}+\chi_{M}\right)\right|-n\right]\left[1-\left(1-P_{0}\right)^{n}\right]
\end{aligned}
$$

To give an idea about the difference between multi-wafer and bulk crystal units numerical values for $\mathrm{P}_{0}, \mathrm{P}_{\mathrm{b}}$ and $\mathrm{P}_{\mathrm{a}}$ are plotted in Figs. $2.1-2.4$ as functions of the cutting angle $\chi_{M}$ for four different reflections of silicon. To each value of $\chi_{M}$ the radius of curvature was assigned that minimizes the linewidth at $90^{\circ}$ detector angle $\left(2 \theta_{\mathrm{S}}\right)$, using relation (2.5). The curve for $\mathrm{P}_{0}$ shows the variation of the peak reflectivity of a single wafer on bending to the corresponding radius, relation (2.9). One should keep in mind that $P_{b}$ refers to a reflectivity curve defined in the phase space of the incident neutrons. In Figs. 2.1-2.2 $\mathrm{P}_{\mathrm{b}}$ reaches the value of a single wafer at positive cutting angles where the teeth of the reflectivity curve merge. However, if one looks at the emergence window in the phase space of the reflected neutrons, this is not a good situation because the value of $P_{a}$ is then low. The average reflectivity $P_{a}$ decreases at large cutting angles $\left(\chi_{M}>0\right.$ range) because the increase of the peak reflectivity $P_{b}$ is not enough to compensate for the expansion of the overall phase space volume after reflection. A similar decrease of $\mathrm{P}_{\mathrm{a}}$ occurs at $\chi_{\mathrm{M}}<0$. A maximum for $\mathrm{P}_{\mathrm{a}}$ is reached around $\chi_{\mathrm{M}} \sim-15^{\circ}$, defining the optimal range of cutting angles. The situation is more complicated in Figs. 2.3-2.4 because of the stronger variation of $P_{0}$ related to lower 
kinematic reflectivities. The gain over the single wafer seen for $\mathrm{P}_{\mathrm{b}}$ at large $\chi_{\mathrm{M}}$ is due to the fact that those neutrons not reflected by the first wafers in the packet (even if the Bragg relation was fulfilled) got a second chance to be reflected by subsequent wafers.

Perhaps more suggestive should be Figs. $2.5-2.8$, where a comparison is made with the bulk crystal case. The thickness of the multi-wafer unit was kept at 1 $\mathrm{cm}$, but the thickness of the reference bulk crystal was set at the breaking limit for every radius of curvature (by using an empirical relation for that limit in silicon) ${ }^{\#}$. The curves marked "peak ratio" show the ratio $P_{2} / P_{0}$, the curves marked " $\Delta \theta$ range ratio" refer to the overall width of the reflectivity curves, and the curves marked "intensity ratio" give the gain in full beam intensities. These gains are seen to be of 3.5 to 4 in all cases considered.

The general conclusion is that for the case of diffraction lines with minimum width at $90^{\circ}$ detector angle, multi-wafer units with moderate cutting angles (from $-20^{\circ}$ to $+15^{\circ}$ ) are definitely better than bulk crystal units.

A possible substitute for $\mathrm{Si}$ in multi-wafer units is Ge. Germanium has better kinematic reflectivity, but stronger absorption for neutrons. Consequently the peak reflectivity formulae must be corrected for absorption. As the lattice parameters for $\mathrm{Ge}$ and $\mathrm{Si}$ are close, it is possible to compare directly the corresponding monochromator performance by an "intensity ratio" for the same reflection. At $1 \mathrm{~cm}$ total thickness the intensity ratio $(\mathrm{Ge} / \mathrm{Si})$ is 0.85 for strong reflections like (400) and (311), 1.24 for (331), and 1.57 for (511). The gain in the case of weak reflections is at the same monochromator dimensions, but commercially available $\mathrm{Ge}$ wafers have smaller diameters $\left(5^{\prime \prime}-127 \mathrm{~mm}\right)$ than $\mathrm{Si}\left(8^{\prime \prime}-20.32 \mathrm{~mm}\right.$ to $\left.12^{\prime \prime}-30.48 \mathrm{~mm}\right)$ and the price is higher by an order of magnitude. 


\subsection{Optimal Configurations}

\section{DAX simulations}

Neutron optics programs for curved crystal spectrometers were worked out after the generic problem was expressed in matrix language [13]. This allowed the extension of the Cooper-Nathans formalism [14] to include spatial effects and bent crystals. The transmission function of the spectrometer $\mathrm{p}\left(\mathbf{k}_{\mathbf{i}}, \mathbf{r}, \mathbf{k}_{\mathbf{f}}\right)$ is extended by simply adding the coordinate $\mathbf{r}$ of the point in the sample where scattering occurs. This function is constructed from the transmission functions of the monochromator and analyzer units $\mathrm{p}_{\mathrm{M}}\left(\mathbf{k}_{\mathrm{i}}, \mathbf{r}\right)$ and $\mathrm{p}_{\mathrm{A}}\left(\mathbf{k}_{\mathbf{f}}, \mathbf{r}\right)$. These are obtained by considering the Bragg reflection on a bent crystal in phase space. Transmission functions are approximated by multi-dimensional Gaussian functions. Covariance matrices of phase space variables are computed and then the covariance matrix of the resolution function variables is obtained through matrix operations [13].

Codes considering both resolution and intensity were developed on this basis, for design and optimization of scattering instruments, as well as for planning experiments. [9]. A program for diffraction arrangements (DAX) was written in Romania under a project supported in part by IAEA Vienna. It has been upgraded at MURR and has been used in the design of monochromator units for neutron powder diffraction, small angle neutron scattering (SANS) and synchrotron radiation (SR). With this program, analytical and numerical optimizations can be done. A figure of merit (ratio of peak intensity at detector to linewidth squared) is maximized numerically. The linewidth refers to powder diffraction.

The DAX program was used to compute monochromator performance (powder diffraction linewidth, flux at sample, beam size at sample, etc.) in different arrangements (distance monochromator to sample, horizontal and vertical curvature of monochromator, cutting angle, etc). A survey of options of interest to ORNL was performed with the idea of having a choice of wavelength for neutron stress mapping of various materials. Because the take-off angle accessible at the new HB-2 beamport

\footnotetext{
" The Si(100) breaking limit of $\mathrm{g}[\mathrm{cm}]=\left(\mathrm{R}_{\mathrm{H}}[\mathrm{cm}] / 2714\right)^{3 / 4}$ was assumed.
} 
is about $90^{\circ}$ and the range of detector angles in residual stress measurements is limited $\left(70^{\circ}-110^{\circ}\right)$, the Bragg reflections (311), (400), (331) and (511) of silicon were chosen for examination. These reflections give suitable wavelengths to measure diffraction patterns in a wide range of d-spacings $(0.09-0.19 \mathrm{~nm})$.

The design dimensions considered in this search were:

- distance from source to monochromator:

$725 \mathrm{~cm}^{*}$

- diameter of the source:

$18 \mathrm{~cm}$

- opening of the in-pile shutter:

$10 \times 10 \mathrm{~cm}$

- monochromator dimensions (width, height):

$16.5 \times 15.2 \mathrm{~cm}$

- distance from monochromator to sample:

$190 \mathrm{~cm}^{*}$

- openings of the reflected beam shutter:

entrance

$7.6 \times 12.7 \mathrm{~cm}$

exit

$3.8 \times 5.1 \mathrm{~cm}$

- sample size:

$1 \times 1 \times 5 \mathrm{~mm}$

- distance from sample to PSD:

$80 \mathrm{~cm}$

- PSD spatial resolution:

$1 \mathrm{~mm}$

For studies on steels using the $\alpha-\mathrm{Fe} 211$ line at detector angles around $90^{\circ}$ one cannot improve upon the existing unit with Si (331) asymmetric $\left(\chi=28.5^{\circ}\right)$ reflcction. A slight intensity gain of $30 \%$ can be obtained by resetting the horizontal curvature from the present value of $R_{H}=10.7 \mathrm{~m}$ to the value of $R_{H}=14.7 \mathrm{~m}$, optimal for the larger distance to sample. However, such a gain will only be available for samples with no intrinsic line broadening, not typical for stressed materials. A small gain (of 7\%) can also be obtained by changing the radius of vertical curvature from $R_{v}=1.55 \mathrm{~m}$ to $R_{v}=1.67 \mathrm{~m}$. The conclusion is that the existing unit already goes to the limit of what silicon can give in high resolution neutron stress profiling at distances to sample around $1.8 \mathrm{~m}$. This is because at the present crystal size and cutting angle, the HB-2 geometry provides a nearly full illumination of the monochromator.

Many options for single reflection monochromators have been compared, including arrangements with transmission geometry and with strong asymmetric reflection geometry in beam condensation (Fankuchen range) or beam extension (inverse Fankuchen). The best solution for multiple choice of wavelengths was found 
to be a multi-wafer unit with $\mathrm{Si}[100]$ orientation and with the $\langle 011\rangle$ zone axis vertical. This unit provides the $\mathrm{Si}(400)$ symmetric reflection and the asymmetric reflections Si (311) and Si (511). At fixed take-off angle, the wavelength is changed simply by rotating the monochromator. On changing the wavelength the horizontal curvature has to be adjusted (by remote control of the adjusting screw). The vertical curvature may be fixed at a value representing a compromise for all wavelengths.

Varying the take-off angle was considered with the aim to adjust to $90^{\circ}$ the detector angle for given reflections from various samples. It was found that such a variation would give only a marginal gain in the figure of merit while greatly complicating the beam extraction and dramatically increasing the cost to implement the solution.

The best results for this series of computations are summarized in Table 2.2 for a take-off angle of $88^{\circ}$. Instrumental linewidths versus detector angle are shown in Figs. $2.9-2.12$ for several take-off angles.

Table 2.2

\begin{tabular}{|c|c|c|c|c|c|c|c|}
\hline Type & $\begin{array}{c}\lambda \\
{[\AA ̊]}\end{array}$ & $\begin{array}{c}\chi \\
\text { [deg.] }\end{array}$ & $\begin{array}{l}R_{H} \\
\text { [m] }\end{array}$ & $\begin{array}{l}\mathrm{R}_{V} \\
{[\mathrm{~m}]}\end{array}$ & $\begin{array}{c}\text { Beam cross } \\
\text { section at sample } \\
\text { [mm] } \\
\text { width } x \text { height }\end{array}$ & $\begin{array}{l}\text { Flux } \\
\text { relative to } \\
\text { single } \\
(331)\end{array}$ & $\begin{array}{c}\text { FWHM } \\
\text { at } \\
2 \theta_{s}=90^{\circ} \\
\text { [deg.] }\end{array}$ \\
\hline Si(311) & 2.27 & 25.3 & 9.5 & 1.75 & $28 \times 21$ & 0.59 & 0.26 \\
\hline $\mathrm{Si}(400)^{2}$ & 1.89 & 0 & 5.1 & 1.75 & $22 \times 21$ & 2.06 & 0.19 \\
\hline Si(331) & 1.73 & 28.5 & 10.7 & 1.55 & $28 \times 21$ & 1 & 0.19 \\
\hline $\operatorname{Si}(511)^{2}$ & 1.45 & 15.8 & 7.4 & 1.75 & $26 \times 21$ & 1.53 & 0.21 \\
\hline
\end{tabular}

1 - Existing Si (331) monochromator

2 - Multi-wafer Si unit with adjustable horizontal curvature and fixed vertical curvature

\section{Phase space diagram simulations}

A useful characterization of neutron monochromators is by their acceptance windows in phase space. These are similar to the acceptance diagrams defined in two

\footnotetext{
* Preliminary design dimensions for the new HB-2.
} 
dimensions $(y, \gamma)$, but are three dimensional, in the phase subspaces $v_{i}=\left(y_{i}, \gamma_{i}, \Delta k / k\right)$ or $\mathbf{v}_{\mathrm{f}}=\left(\mathrm{y}_{\mathrm{f}}, \gamma_{\mathrm{f}}, \Delta \mathrm{k} / \mathrm{k}\right)$. With flat crystals the spatial coordinate does not matter and the windows are two-dimensional, depicted in the well-known DuMond $(\Delta \lambda / \lambda, \gamma)$ diagrams [15].

In neutron diffraction strain mapping the gauge cross section of the beam is usually $2 \times 2 \mathrm{~mm}$ (or less). This makes it possible to describe the monochromator in terms of two-dimensional angle-wavelength diagrams instead of full three-dimensional diagrams. Such diagrams correctly describe the situation at the beam center only, but the representation is free of the errors introduced by Gaussian approximations. The matrix expressions given in the previous two chapters were used to compute the diagrams. The diffraction line widths in a $2 \theta_{\mathrm{s}}$ range of $70^{\circ}-110^{\circ}$ were computed by taking into account the contribution of spatial beam spread on the acceptance diagram and the contribution of angular collimation after sample. From computed variances, linewidths were estimated by assuming diffraction profiles to be Gaussian.

Table 2.3

\begin{tabular}{|c|c|c|c|c|c|c|c|c|}
\hline Type & $\begin{array}{c}\chi \\
{[\text { deg.] }}\end{array}$ & $\begin{array}{c}\mathrm{R}_{\mathrm{H}} \\
{[\mathrm{m}]}\end{array}$ & $\begin{array}{c}\mathrm{R}_{\mathrm{V}} \\
{[\mathrm{m}]}\end{array}$ & $\begin{array}{c}\Delta \gamma \\
{\left[{ }^{\circ}\right]}\end{array}$ & $\begin{array}{c}\Delta \delta \\
{\left[{ }^{\circ}\right]}\end{array}$ & $\mathrm{P}_{\mathrm{a}}$ & $\begin{array}{c}\text { Flux } \\
\text { relative to } \\
\text { single } \\
(331)\end{array}$ & $\begin{array}{c}\text { FWHM } \\
\text { at } \\
2 \theta_{s}=90^{\circ} \\
{[\mathrm{deg} .]}\end{array}$ \\
\hline (311)bulk & 25.3 & 11.4 & 1.6 & 0.9 & 3.6 & 0.98 & 0.6 & 0.21 \\
\hline$(311)$ multi & 25.3 & 11 & $1.4-1.8$ & 0.9 & 3.6 & 0.40 & 0.6 & 0.27 \\
\hline (400)multi & 0 & $5.2-5.5$ & $1.5-2.0$ & 2.0 & 3.6 & 0.64 & 2.7 & 0.24 \\
\hline (331)bulk & 28.5 & 10.7 & 1.55 & 0.6 & 2.5 & 0.69 & 1 & 0.30 \\
\hline (331)bulk & 28.5 & $13.4-13.8$ & $1.3-1.7$ & 0.7 & 3.6 & 0.86 & 1 & 0.21 \\
\hline (511)multi & 15.8 & $7.7-7.8$ & $1.5-2.0$ & 1.4 & 3.6 & 0.35 & 2.1 & 0.26 \\
\hline
\end{tabular}

* - Existing Si (331) monochromator in present HB-2 design.

The phase space diagrams for the optimal monochromator configurations mentioned before are shown in Figs. 2.13 - 2.16. The optimal radii of curvature and the corresponding performances of the monochromator are given in Table 2.3. The wavelength is identical with that of Table 2.2 as the take-off angle was the same $\left(88^{\circ}\right)$. 
The design dimensions considered in the survey were afterwards modified to more realistic values:

- distance from source to monochromator:

- diameter of the source:

- opening of the in-pile shutter:

- monochromator dimensions:

- distance from monochromator to sample: $768 \mathrm{~cm}^{*}$

- openings of the reflected beam shutter:

entrance $18 \mathrm{~cm}$ exit $9 \times 9 \mathrm{~cm}$ $16.5 \times 15.2 \mathrm{~cm}$ $212 \mathrm{~cm}^{*}$

- slits before and after sample:

- distance from sample to PSD:

- PSD spatial resolution:

$\begin{array}{ll} & 768 \mathrm{~cm}^{*} \\ & 18 \mathrm{~cm} \\ & 9 \times 9 \mathrm{~cm} \\ & 16.5 \times 15.2 \mathrm{~cm} \\ & 212 \mathrm{~cm}^{*} \\ \text { entrance } & 7.6 \times 12.7 \mathrm{~cm} \\ \text { exit } & 7.6 \times 12.7 \mathrm{~cm} \\ & 2 \times 2 \mathrm{~mm} \\ & 80 \mathrm{~cm}^{\#} \\ & 2 \mathrm{~mm}^{\#}\end{array}$

The focusing in scattering for the case of powder diffraction can be expressed in terms of phase space diagrams. For a broad neutron beam converging onto a small sample, the diffraction line is still narrow if the combination $k \sin \theta_{S}$ is kept constant $\left(\mathrm{Q}=2 \mathrm{k} \sin \theta_{\mathrm{s}}-\right.$ the scattering vector, $2 \theta_{\mathrm{S}}-$ the detector angle). This can be done through a tight correlation between $\mathrm{k}$ and the direction of the neutrons in the incident beam. The necessary correlation is ensured by a correct curvature of the crystal, given by the relation (2.4), and occurs when the needle shaped $(\Delta \mathrm{k} / \mathrm{k}, \gamma)$ phase space diagram is inclined by an angle $\alpha_{S}$ relative to the $\Delta \mathrm{k} / \mathrm{k}$ axis satisfying the relation:

$$
\tan \alpha_{\mathrm{s}}=-2 \tan \theta_{\mathrm{s}}
$$

For $2 \theta_{S}=90^{\circ}$ one gets $\alpha_{S}=-63.434^{\circ}$. All acceptance diagrams should thus have the same orientation regardless of the wavelength and geometrical configuration.

Figures 2.13-2.16 illustrate this principle. In Fig. 2.14, which refers to the multi-wafer monochromator, the thickness of the needle in the phase space diagram is larger that in other cases. It was for this reason that we added in Table 2.3 the case of (311) bulk monochromator: the bulk option is better (better resolution at the same intensity). This is a general finding: at strong asymmetries with beam condensation (Fankuchen range) bulk monochromators are better than multi-wafer monochromators.

\footnotetext{
* Revised new HB-2 design dimensions.
} 
For symmetric or nearly symmetric reflection a multi-wafer monochromator has a larger angular acceptance and, in spite of the lower peak reflectivity, a better extraction efficiency (defined by the effective phase space volume selected by monochromator from source).

The last column in table 2.3 contains the linewidths at $2 \theta_{\mathrm{S}}=90^{\circ}$. For multiwafer units linewidths are larger. This is due to a larger effective thickness (by a factor of about 2). For stressed samples with intrinsic line broadening this difference should not be significant. The linewidth variation with detector angle is displayed in Fig. 2.17. The results are consistent with DAX computations.

Relative figures of merit of different monochromators are plotted in Fig. 2.18 for three values of the microstrain $(0,0.002,0.004)$, the primary factor in the sample contribution to line broadening. The figure of merit was defined in section 1.2, relation (1.7). The figure of merit of the present $\mathrm{Si}$ (331) bulk crystal unit on measuring the (211) line of $\alpha$-iron without microstrain was taken as reference (figure of merit $=1$ ). The d-spacing scale is independent of the choice of the wavelength. Different monochromators can thus be compared directly. The d-spacings frequently used in stress measurements on various materials are indicated. Figure 2.18 is useful for the selection of the most suitable monochromator at given diffraction lines of stressed materials. The conclusion is that to cover the whole range of $d$-spacings required in residual strain measurements at least three monochromator units are necessary: one multi-wafer and two of the bulk crystal type.

In the configurations examined above it was assumed that the multi-wafer unit is made from commercially available $\mathrm{Si}(100)$ wafers. This results in a limitation of the performance of multi-wafer units. The next section will consider the situation when the wafers are allowed to have optimal crystallographic orientations. This allows the achievement of maximum benefit from using multi-wafer monochromators.

\footnotetext{
\# Selected to assure predicted FWHM agree with measured FWHM for existing system.
} 


\subsection{Recommended Configuration}

To optimize the figure of merit of multi-wafer monochromators we looked for wafer orientations around the [100], [111] and [110] directions. For the reflections (400), (511) and (311) the best choice of orientation is a moderate inclination $\left(10^{\circ}-15^{\circ}\right)$ of the normal to the surface from the [100] direction toward the [011] direction. The best choice for (331) reflection is just the [110] wafer orientation. The total unit thickness was also optimized. The best average figure of merit was obtained for total crystal thickness of $1.25 \mathrm{~cm}$ (18 wafers). The basic performance is summarized in Table 2.4 and the corresponding figures of merit are given in fig. 2.19. An average increase of $50 \%$ in figure of merit is obtained in comparison with the configurations considered previously for (400), (311), and (511) reflections, but more than $100 \%$ increase for (331).

In computations the horizontal curvature was fixed at the value corresponding to best linewidths at $2 \theta_{\mathrm{S}}=90^{\circ}$. By making the curvature variable to optimize the resolution at every scattering angle one can further improve the figure of merit. The dependence of linewidths on detector angle for the options of fixed and variable radius of curvature are compared in figs. 2.20-2.23. The figures of merit at variable radius are shown in fig. 2.24. Figures of merit increase at high detector angle (small $d_{s}$ ) because optimal radii decrease there. Consequently, not only the linewidth improves on going to the optimal radius, but also the intensity increases.

Table 2.4

\begin{tabular}{|c|c|c|c|c|c|c|c|c|}
\hline Type & $\begin{array}{c}\chi \\
{[\text { deg.] }}\end{array}$ & $\begin{array}{c}\mathrm{R}_{\mathrm{H}} \\
{[\mathrm{m}]}\end{array}$ & $\begin{array}{c}\mathrm{R}_{\mathrm{V}} \\
{[\mathrm{m}]}\end{array}$ & $\begin{array}{c}\Delta \gamma \\
{\left[{ }^{\circ}\right]}\end{array}$ & $\begin{array}{c}\Delta \delta \\
{\left[{ }^{\circ}\right]}\end{array}$ & $\mathrm{P}_{\mathrm{a}}$ & $\begin{array}{c}\text { Flux } \\
\text { relative to } \\
\text { single } \\
(331)\end{array}$ & $\begin{array}{c}\text { Fwhm at } \\
2 \theta_{\mathrm{s}}=90^{\circ} \\
{[\mathrm{deg} .]}\end{array}$ \\
\hline (311)multi & 13.2 & 7.8 & 1.75 & 1.6 & 3.6 & 0.61 & 1.9 & 0.29 \\
\hline (400)multi & -12 & 5.0 & 1.75 & 2.0 & 3.6 & 0.90 & 3.5 & 0.23 \\
\hline (331)multi & -13.3 & 4.9 & 1.75 & 2.0 & 3.6 & 0.61 & 2.7 & 0.23 \\
\hline (511)multi & 3.8 & 6.4 & 1.75 & 2.0 & 3.6 & 0.33 & 2.9 & 0.27 \\
\hline
\end{tabular}


By adjusting the horizontal curvature and by choosing the monochromator that gives the largest detector angle (below $2 \theta_{\mathrm{s}}=110^{\circ}$ ) for each material, figure of merit gains from 3 to 10 can be reached at sample d-spacings from 0.09 to $0.15 \mathrm{~nm}$.

Finally one can conclude that for the single reflection monochromator (SRM) option two variable horizontal radius multi-wafer units with wafers of non-standard orientation and with the following characteristics will optimize the new HB-2 neutron instrument for mapping stresses in a wide variety of materials and for measurements of multiple lines $\left(h_{1} k_{1} l_{1} ; h_{2} k_{2} l_{2} ; \ldots\right)$ of a given sample:

1. SRM option A - used for (311), (400) and (511) reflections:

- silicon wafer orientation: $12^{\circ}$ from [100] toward [011];

- wafer thickness: $\sim 0.7 \mathrm{~mm}$;

- wafer vertical segmentation : $-5 \mathrm{~mm}$;

- total crystal thickness: $\sim 1.25 \mathrm{~cm}$;

- remotely adjustable radius of horizontal curvature from $2 \mathrm{~m}$ to $14 \mathrm{~m}$;

- fixed radius of vertical curvature: $1.75 \mathrm{~m}$.

2. SRM option B - used for (331) reflection:

- silicon wafers orientation: [110];

- wafer thickness: $\sim 0.7 \mathrm{~mm}$;

- wafer vertical segmentation : $5 \mathrm{~mm}$;

- total crystal thickness: $\sim 1.25 \mathrm{~cm}$;

- remotely adjustable radius of horizontal curvature from $5 \mathrm{~m}$ to $14 \mathrm{~m}$;

- fixed radius of vertical curvature: $1.75 \mathrm{~m}$.

The dimensions of the bending device considered in these estimates are the same as those of the existing $\mathrm{Si}$ (331) bulk focusing monochromator. The two recommended units can be mounted on the same $\mathrm{XY} \psi$ monochromator stage. The remotely controlled translation $X$ permits adjusting the working unit to the rotation center of the goniometer. The remotely controlled translation $\mathrm{Y}$ pcrmits left-right centering of the monochromator over the rotation center. The $\psi$ rofation turns the entire monochromator unit to achieve the desired $2 \theta_{\mathrm{M}}$. 


\section{DOUBLE REFLECTION MONOCHROMATOR}

\subsection{Double crystal focusing conditions}

Double crystal arrangements are widely used in monochromators for synchrotron radiation, but only with flat crystals or sagitally curved crystals. The first analysis of two-bent-crystal Bragg optics came from neutron scattering [11]. Subsequently the analysis was extended to synchrotron radiation [16].

For single reflection monochromators the tracing of the trajectories and the dispersive properties (the associate wavevectors) can be treated separately. In double monochromators only trajectories giving the same wavevector on successive Bragg reflections are allowed. For double reflection of high efficiency the phase space diagrams of the two bent crystals must be matched. In the general case two matching conditions must be satisfied [11], but only one condition applies if a point source (or a point object) is considered [16].

For a point source located at distance $L_{0}$ before the first crystal, the image after reflection by the first crystal will be placed at a distance $\mathrm{L}_{\mathrm{i}}$ :

$$
\frac{f_{1 e}}{L_{0}}+\frac{f_{1 e}^{*}}{L_{i}}=1
$$

The definitions for $f_{1 \mathrm{c}}$ and $\mathrm{f}_{1 \mathrm{c}}{ }^{*}$ were given in section 2.1, relations 2.2a,b. A similar relation is valid for the virtual point source placed at the distance $L_{1}-L_{i}$ before the second crystal and giving a second image at a distance $\mathrm{L}_{2}$ after it:

$$
\frac{f_{2 e}}{L_{1}-L_{i}}+\frac{f_{2 e}^{*}}{L_{2}}=1
$$

$\mathrm{L}_{1}$ being the distance between the two crystals.

Matching conditions are obtained by requiring the orientations of the phase space diagrams of both crystals to be identical:

$$
\cot \theta_{1}\left(1-\frac{L_{i}}{2 f_{1}^{*}}\right)=-\cot \theta_{2}\left(1-\frac{L_{1}-L_{i}}{2 f_{2}}\right)
$$

This relation may be brought to the form [16]: 


$$
a \frac{L_{i}}{f_{1}^{*}}-\frac{L_{1}-L_{i}}{f_{2}}=2(a-1)
$$

where $\mathrm{a}=-\tan \theta_{2} / \tan \theta_{1}$

Consider the spatial spread of the neutron beam at the point $\mathrm{L}_{\mathrm{i}}$. To match the correlation between the spatial position and the angular deviation (the acceptance diagrams) the following condition must be fulfilled:

$$
f_{2}+f_{1}^{*}=\frac{L_{1}}{2}
$$

By combining the relations (3.3') and (3.4), the basic focal length conditions for an extended source non-dispersive ${ }^{*}$ two-crystal configuration are obtained [11]:

$$
\begin{aligned}
& f_{1}^{*}=\frac{a L_{1}}{2(a-1)} \\
& f_{2}=\frac{L_{1}}{2(1-a)}
\end{aligned}
$$

For given crystals and distances between crystals, the focal lengths are fixed. A special case is the $(+,-)$ setting with identical crystals, when $a=1$ and $f_{1}{ }^{*}, f_{2} \rightarrow \infty$. This is the parallel setting for flat crystals, well known to be non-dispersive.

The antiparallel $(+,+)$ setting of two bent crystals turns out to have a remarkable property of allowing strong spatial focusing, named microfocusing $[17,18]$. For equal d-spacings $(a=-1)$ :

$$
\begin{aligned}
& f_{2}=f_{1}^{*}=\frac{L_{1}}{4} \\
& \frac{b_{2} L_{2}}{L_{1}}=\frac{3-\frac{b_{1} L_{1}}{L_{0}}}{8-3 \frac{b_{1} L_{1}}{L_{0}}}
\end{aligned}
$$

where $b_{1}, b_{2}$ are the asymmetry factors $\sin \left(\theta_{j}+\chi_{j}\right) / \sin \left(\theta_{j}-\chi_{j}\right)$. The demagnification of the lens system in the horizontal plane (ratio between the beam width at sample position and at source) is given by the formula:

\footnotetext{
* The condition that all neutrons reflected by the first crystal are reflected also by the second crystal.
} 


$$
M_{e}=\frac{1}{b_{1} b_{2}} \times \frac{\frac{b_{1} L_{1}}{L_{0}}}{8-3 \frac{b_{1} L_{1}}{L_{0}}}
$$

The horizontal demagnification of arrangements with identical crystals may be smaller by an order of magnitude than for single reflection arrangements $\left(M_{e}=L_{1} / L_{0}\right)$. The neutron flux is correspondingly higher. The flux gain is associated with a larger angular divergence of the beam at the sample, which is finally determined by the dimensions of the second crystal and the distance to sample $\mathrm{L}_{2}$. The gain reaches its maximum value of $1 / \mathrm{M}_{\mathrm{e}}$ when the second crystal is large enough to intercept the whole beam from the first crystal.

To have a narrow powder diffraction line at a scattering angle $2 \theta_{\mathrm{S}}$ one has to satisfy the condition (2.5). Leting as $=-\tan \theta_{\mathrm{S}} / \tan \theta_{2}$, the condition (2.5) becomes:

$$
\frac{L_{2}}{f_{2}^{*}}=2-\frac{1}{a_{S}}
$$

Using the relations (3.6-3.8) one obtains the demagnification as function of as:

$$
M_{e}=\frac{1-\frac{2}{a_{s}}}{b_{1} b_{2}}
$$

With identical crystals in $(+,+)$ setting a value of as close to 2 will ensure strong demagnification. The diffraction lines will be narrow around $90^{\circ}$ if the take-off angle of the crystals is close to $53^{\circ}$ [17].

The value of $a_{s}$ will determine not only the optimal detector angle but also the entire diffractometer geometry:

$$
\begin{aligned}
& \frac{b_{2} L_{2}}{L_{1}}=\frac{2 a_{s}-1}{4 a_{s}} \\
& \frac{b_{1} L_{1}}{L_{0}}=\frac{4\left(a_{s}-2\right)}{2 a_{s}-3}
\end{aligned}
$$

When $a_{s}=2$ the double reflection monochromator acts like a telescope $\left(L_{0} \rightarrow \infty\right)$. However, having the source image at the sample position is less important than having 
narrow diffraction lines at a specified detector angle. For this reason the relation (3.11) will not be an imposed constraint in the following.

Similar relations are available in the general case $(a \neq-1)$ :

$$
\begin{aligned}
& \frac{b_{2} L_{2}}{L_{1}}=\frac{2-a+a \frac{b_{1} L_{1}}{L_{0}}}{2(1-a)^{2}-a(1-2 a) \frac{b_{1} L_{1}}{L_{0}}} \\
& M_{e}=\frac{1}{b_{1} b_{2}} \times \frac{-a \frac{b_{1} L_{1}}{L_{0}}}{2(1-a)^{2}-a(1-2 a) \frac{b_{1} L_{1}}{L_{0}}} \\
& M_{e}=\frac{1+\frac{1-a}{a a_{s}}}{b_{1} b_{2}}
\end{aligned}
$$

$$
\frac{b_{2} L_{2}}{L_{1}}=\frac{1-\frac{1}{2 a_{S}}}{1-a}
$$

$$
\frac{b_{1} L_{1}}{L_{0}}=\left(1-\frac{1}{a}\right) \frac{1+\frac{1-a}{a a_{s}}}{1+\frac{1-2 a}{2 a a_{s}}}
$$

The optimal value for as in the general case is as $=1-1 / \mathrm{a}$. By considering the Bragg angle of the first crystal $\left(\theta_{1}\right)$ one can define $a^{*}{ }^{*}=-\tan \theta_{S} / \tan \theta_{1}$ or as ${ }^{*}=-$ a $\times$ as. The optimal value for as ${ }^{*}$ is $1-\mathrm{a}$, which means that larger take-off angles for the first crystal are possible when $|a|<1$.

In the vertical (axial) plane the successive reflections are not affected in the first approximation by the Bragg law and the crystals are simple mirrors. At fixed distances $L_{0}, L_{1}$, and $L_{2}$ one relation only between the axial focal lengths $f_{a 1}, f_{a 2}$ - see relation (2.4) for the definition - must be satisfied: 


$$
\frac{L_{2}}{f_{a 2}}=\frac{1+\frac{L_{1}+L_{2}}{L_{0}}-\frac{L_{1}+L_{2}}{f_{a 1}}}{1+\frac{L_{1}}{L_{0}}-\frac{L_{1}}{f_{a 1}}}
$$

The axial demagnification will be determined by the formula:

$$
M_{a}=\frac{-\frac{L_{2}}{L_{0}}}{1+\frac{L_{1}}{L_{0}}-\frac{L_{1}}{f_{a 1}}}
$$

and if the first crystal is vertically flat the axial demagnification is approximately the ratio between $L_{2}$ and $L_{0}$, which is much smaller than unity.

The flux gain at the sample from vertical focusing is limited by the second crystal height. If both crystals are equally high then the second crystal will be fully illuminated by the neutron beam only when:

$$
\mathrm{f}_{\mathrm{a} 1}>\mathrm{L}_{0} \text { or } \mathrm{f}_{\mathrm{a} 1}<\mathrm{L}_{1} /\left(2+\mathrm{L}_{1} / \mathrm{L}_{0}\right) \text {. }
$$

If (3.14) is satisfied the flux gain produced by vertical focusing will be the same at any curvatures satisfying (3.12).

\subsection{Double reflection with identical crystals}

The case of identical bent crystals in double reflection was examined [17]. For strain measurements with the $\mathrm{Fe}$ (211) line the best double reflection monochromator was found to be $\mathrm{Cu}(200)$ of small mosaic ( $3^{\prime}$ true), deformed by plastic bending. Although such monochromators are still in the development stage, it was this option that was taken as basis for double reflection simulations.

The DAX program was used to compute linewidths and peak intensities (relative to the existing single reflection monochromator) versus detector angle, full beam intensity, flux and beam size at sample. The distance $L_{1}$ was chosen by taking into account the real configuration at the HB-2 beamport and $L_{2}$ was then calculated through relation (3.10). The radii of curvature were selected by numerical optimization. Five basic double crystal options considered were: $\mathrm{Cu}$ (200) in 
symmetric reflection; $\mathrm{Si}(220)$ in symmetric reflection and with $\chi=15^{\circ} ; \mathrm{Si}(311)$ with $\chi=21^{\circ}$; Si (400) in symmetric reflection.

The crystal size for $\mathrm{Cu}(200)$ was $7.6 \mathrm{~cm}$ wide, $12.7 \mathrm{~cm}$ high and $2.5 \mathrm{~mm}$ thick. An effective mosaic of 0.1 degrees was considered in simulations. For silicon the multi-wafer options are necessary to achieve the high curvature that is required. To obtain decent resolution the second crystal was set to $2.2 \mathrm{~mm}$ thick ( 3 wafers). The first unit was assumed to be $10 \mathrm{~mm}$ thick (14 wafers). The reason of this difference is for the first crystal to have a broader phase space window. For perfect crystals like silicon the width of the phase space window is fully determined by the crystal thickness, thus a larger thickness is needed to broaden the window. For mosaic crystals it is the mosaic contribution that is usually decisive and increasing the thickness is not effective (particularly for $\mathrm{Cu}$ ) due to the influence of absorption.

The main results of DAX simulations are summarized in Tables 3.1 and 3.2. Table 3.1 contains the basic performances of each of the double reflection arrangements examined. The last two columns require some explanations. The first of them contains the angular $2 \theta_{s}$ range inside which the instrumental linewidth is smaller than $0.5^{\circ}$. The last column contains the harmonics contamination (fraction of neutrons having a wavelength half of the wavelength corresponding to the main reflected beam). The flux gains and beam sizes are impressive.

However, a simple evaluation shows that the maximum possible gain in the beam divergence solid angle is only $16 \times$ for the $\mathrm{Cu}(200)$ double reflection arrangement relative to the $\mathrm{Si}$ (331) single reflection. The larger values in Table 3.1 are at the expense of broadening the phase space windows, which increases linewidths too. Not only are the linewidths at $2 \theta_{S}=90^{\circ}$ larger but the linewidth variation is much sharper in comparison with single reflection options. To illustrate this point Fig. 3.1 gives a map of linewidths as a function of take-off angle and sample d-spacing for double reflection $\mathrm{Cu}(200)$. The optimum radii for a take-off angle of $53^{\circ}$ were considered in this evaluation. It is seen that measurements with a resolution of $0.35^{\circ}$ $0.40^{\circ}$ are possible in a narrow range of $\mathrm{d}$-spacings (indicated by the thick lines corresponding to detector angles of $80^{\circ}$ and $100^{\circ}$. Changing the take-off angle does not help because the flux rapidly decreases on going away from the focusing condition. 
The silicon option is more flexible from this point of view, but the intensity gains are not so large.

Table 3.2 contains the optimum parameters of the double reflection arrangements considered. Two possibilities for the vertical curvatures are shown: both crystals with the same radius or the first crystal flat and only the second bent. Both possibilities give about the same intensity as already noticed in the previous section (3.14).

Table 3.1

\begin{tabular}{|c|c|c|c|c|c|c|c|}
\hline Type & $\begin{array}{c}2 \theta_{1} \\
\vdots \\
\text { [deg.] }\end{array}$ & $\begin{array}{c}\lambda \\
{[\AA \AA]}\end{array}$ & $\begin{array}{c}\text { Beam } \\
\text { cross } \\
\text { section } \\
{[\mathrm{mm}]}\end{array}$ & $\begin{array}{c}\text { Flux } \\
\text { relative } \\
\text { to } \\
\text { single } \\
\text { (331) }\end{array}$ & $\begin{array}{c}\text { FWHM } \\
\text { at } \\
2 \theta_{s}=90^{\circ} \\
\text { [deg.] }\end{array}$ & $\begin{array}{c}0.5^{\circ} \\
\text { width } \\
\text { range } \\
\text { [deg.] }\end{array}$ & $\begin{array}{c}\text { 2-nd } \\
\text { order } \\
\text { contami } \\
\text {-nation } \\
\text { [\%] }\end{array}$ \\
\hline $\mathrm{Cu}(200)$ & 57.2 & 1.73 & $3 \times 5$ & 32 & 0.31 & $73-97$ & 10 \\
\hline $\mathrm{Cu}(200)$ & 57.2 & 1.73 & $3 \times 3$ & 30 & 0.31 & $73-97$ & 10 \\
\hline $\mathrm{Cu}(200)$ & 53 & 1.61 & $3 \times 3$ & 36 & 0.43 & $69-93$ & 10 \\
\hline Si(220) & 57.2 & 1.82 & $3 \times 5$ & 15 & 0.31 & $80-98$ & 2 \\
\hline Si(220) & 53 & 1.71 & $4 \times 3$ & 19 & 0.31 & $83-95$ & 2 \\
\hline Si(220) & 53 & 1.71 & $4 \times 3$ & 24 & 0.31 & $81-97$ & 2 \\
\hline Si $(400)$ & 53 & 1.21 & $4 \times 3$ & 5 & 0.34 & $85-94$ & - \\
\hline Si(311) & 53 & 1.46 & $4 \times 5$ & 12 & 0.40 & $75-96$ & - \\
\hline
\end{tabular}

Table 3.2

\begin{tabular}{|c|c|c|c|c|c|c|c|}
\hline Type & $\begin{array}{c}\mathbf{L}_{\mathbf{1}} \\
{[\mathrm{m}]}\end{array}$ & $\begin{array}{c}\chi_{1} \\
\text { [deg.] }\end{array}$ & $\begin{array}{c}\mathbf{R}_{\mathbf{H} 1} \\
{[\mathbf{m}]}\end{array}$ & $\begin{array}{c}\mathbf{R}_{\mathbf{H} 2} \\
{[\mathbf{m}]}\end{array}$ & $\begin{array}{c}\mathbf{R}_{\mathbf{V} 1} \\
{[\mathbf{m}]}\end{array}$ & $\begin{array}{c}\mathbf{R}_{\mathbf{V} 2} \\
{[\mathbf{m}]}\end{array}$ & $\begin{array}{c}\mathbf{L}_{\mathbf{2}} \\
{[\mathrm{m}]}\end{array}$ \\
\hline $\mathrm{Cu}(200)$ & 1.64 & 0 & 1 & 1.75 & $\infty$ & 0.53 & 0.62 \\
\hline $\mathrm{Cu}(200)$ & 1.64 & 0 & 1 & 1.75 & 0.40 & 0.39 & 0.62 \\
\hline $\mathrm{Cu}(200)$ & 1.80 & 0 & 1.4 & 2.1 & 0.40 & 0.40 & 0.69 \\
\hline $\mathrm{Si}(220)$ & 1.64 & 0 & 2.5 & 1.75 & $\infty$ & 0.53 & 0.62 \\
\hline $\mathrm{Si}(220)$ & 1.80 & 0 & 2.3 & 2.04 & 0.40 & 0.40 & 0.69 \\
\hline $\mathrm{Si}(220)$ & 1.80 & 15 & 3.8 & 2.04 & 0.40 & 0.40 & 0.69 \\
\hline $\mathrm{Si}(400)$ & 1.80 & 0 & 2.3 & 2.04 & 0.40 & 0.40 & 0.69 \\
\hline $\mathrm{Si}(311)$ & 1.64 & 21 & 5 & 2.7 & 0.40 & 0.36 & 0.82 \\
\hline
\end{tabular}


From the point of view of design constraints at HB-2 the double reflection monochromator with identical crystals would need a special shutter to extract the monochromatic beam after the first crystal. This shutter must be inclined at about $55^{\circ}$ to the shield wall. In these circumstances for the single reflection option the distance between monochromator and sample stays about $2.9 \mathrm{~m}$ and the flux at the sample decreases with a factor of about 2.5 from geometrical considerations. For this reason this single reflection configuration was not analyzed in the previous chapter.

\subsection{Double reflection with different crystals}

For neutron microfocusing (quasi-telescopic arrangement) with different crystals optimum take-off angles, corresponding to the canceling of $\mathrm{M}_{\mathrm{e}}$, are given by the relation (3.9'). For the detector angle $2 \theta_{S}=90^{\circ}$, the necessary take-off angles are given in Fig. 3.2 versus the ratio between $d$-spacings of the two reflections $\left(d_{1} / d_{2}\right)$. The ratio between $L_{2}$ and $L_{1}$ is then given by a simple formula:

$$
\frac{b_{2} L_{2}}{L_{1}}=\frac{2-a}{2(1-a)^{2}}
$$

and the radii of curvature can be deduced from relations (3.5). The numerical results are shown in Fig. 3.3. It is seen that the microfocusing conditions are very restrictive and do not allow large take-off angles for the first crystal.

These restrictions can be overcome by assuming that the sample can be considered point-like for strain measurements. The synchrotron radiation matching condition (3.3) can then be used. Ratio $b_{2} L_{2} / L_{1}$ becomes then free. The radius of the second crystal results from relation (3.8) and the matching radius of the first crystal results from the following relation:

$$
\frac{f_{1}^{*}}{L_{1}}=\frac{1-\frac{f_{2}}{L_{2}}-\frac{f_{2}^{*}}{L_{1}}}{2-\frac{1}{a}-2\left(1-\frac{1}{a}\right) \frac{f_{2}^{*}}{L_{2}}}
$$

This relation is independent of the value of as. If the first crystal is thicker than the second, its radius of curvature may be different from the value prescribed by the relation (3.16). The only problem is that the second crystal must be fully illuminated 
by the neutron beam. The condition for a full illumination can be written also in a general form:

$$
R_{2}>\frac{2 L_{1}}{\sin \left(\theta_{1}-\chi_{1}\right)+\frac{L_{1}}{L_{2}} \sin \left(\theta_{2}-\chi_{2}\right)+\sin \left(\theta_{2}+\chi_{2}\right)}
$$

where $R_{2}$ is the horizontal radius of curvature for the second crystal.

The DAX program was used as a first step in computing arrangements with two different crystals. A take-off angle of $86.5^{\circ}$ for the first crystal was considered with the aim of preserving the single reflection options. The reflections (311), (400), (331), (422) and (511) of silicon were examined for the first crystal. The d-spacing of the second reflection must be larger than that of the first reflection. The options considered for the second reflection were $\mathrm{Cu}(200), \mathrm{Si}(220)$ and $\mathrm{Si}(400)$. The results are summarized in Table 3.3 and 3.4. Flux gains are less than for identical double reflections, but instrumental linewidths are comparable with the single reflection option. The optimum values of the crystal radii and of the distance between the second monochromator and sample are presented in Table 3.4. First crystals are in beam condensation setting (Fankuchen) and second crystals are in symmetrical reflection.

Table 3.3

\begin{tabular}{|c|c|c|c|c|c|c|c|}
\hline I & \| & $\begin{array}{c}\lambda \\
{[\AA]}\end{array}$ & $\begin{array}{c}\text { Beam } \\
\text { cross } \\
\text { section } \\
{[\mathrm{mm}]}\end{array}$ & $\begin{array}{c}\text { Flux } \\
\text { relative } \\
\text { to } \\
\text { single } \\
(331)\end{array}$ & $\begin{array}{c}\text { FWHM } \\
\text { at } \\
2 \theta_{s}=90^{\circ} \\
\text { [deg.] }\end{array}$ & $\begin{array}{c}0.5^{0} \\
\text { width } \\
\text { range } \\
\text { [deg.] }\end{array}$ & $\begin{array}{c}\text { 2-nd } \\
\text { order } \\
\text { contami } \\
\text {-nation } \\
{[\%]}\end{array}$ \\
\hline Si (311) & $\mathrm{Si}(220)$ & 2.24 & $5 \times 3$ & 8 & .29 & $73-107$ & - \\
\hline Si $(400)$ & $\mathrm{Cu}(200)$ & 1.86 & $3 \times 6$ & 4 & .15 & $65-111$ & 23 \\
\hline $\mathrm{Si}(331)$ & Si(220) & 1.71 & $3 \times 3$ & 10 & .24 & $62-104$ & - \\
\hline $\mathrm{Si}(331)$ & $\mathrm{Cu}(200)$ & 1.71 & $3 \times 7$ & 12 & .20 & $58-108$ & - \\
\hline $\mathrm{Si}(331)$ & $\mathrm{Si}(400)$ & 1.71 & $5 \times 5$ & 9 & .24 & $65-110$ & - \\
\hline $\mathrm{Si}(422)$ & $\mathrm{Cu}(200)$ & 1.52 & $3 \times 8$ & 16 & .31 & $45-104$ & 10 \\
\hline $\mathrm{Si}(422)$ & $\mathrm{Si}(400)$ & 1.52 & $3 \times 6$ & 14 & .25 & $72-103$ & 3 \\
\hline Si(511) & $\mathrm{Si}(400)$ & 1.43 & $3 \times 3$ & 6 & .17 & 68-105 & - \\
\hline
\end{tabular}


Table 3.4

\begin{tabular}{|c|c|c|c|c|c|c|c|}
\hline I & II & $\begin{array}{c}\chi_{\mathbf{1}} \\
{[\mathbf{d e g}]}\end{array}$ & $\begin{array}{c}\mathbf{R}_{\mathbf{H} 1} \\
{[\mathrm{~m}]}\end{array}$ & $\begin{array}{c}\mathbf{R}_{\mathbf{H} 2} \\
{[\mathbf{m}]}\end{array}$ & $\begin{array}{c}\mathbf{R}_{\mathbf{V} 1} \\
{[\mathbf{m}]}\end{array}$ & $\begin{array}{c}\mathbf{R}_{\mathbf{V} 2} \\
{[\mathbf{m}]}\end{array}$ & $\begin{array}{c}\mathbf{L}_{\mathbf{2}} \\
{[\mathbf{m}]}\end{array}$ \\
\hline $\mathrm{Si}(311)$ & $\mathrm{Si}(220)$ & 25.3 & 4.5 & 1.8 & 0.51 & 0.51 & 0.66 \\
\hline $\mathrm{Si}(400)$ & $\mathrm{Cu}(200)$ & 0 & 5.5 & 2.1 & $\infty$ & 0.69 & 0.76 \\
\hline $\mathrm{Si}(331)$ & $\mathrm{Si}(220)$ & 30.5 & 8 & 2.6 & .47 & 0.47 & 0.87 \\
\hline $\mathrm{Si}(331)$ & $\mathrm{Cu}(200)$ & 30.5 & 10.7 & 2.4 & $\infty$ & 0.68 & 0.84 \\
\hline $\mathrm{Si}(331)$ & $\mathrm{Si}(400)$ & 30.5 & 4.5 & 1.6 & $\infty$ & 0.65 & 0.58 \\
\hline $\mathrm{Si}(422)$ & $\mathrm{Cu}(200)$ & 35.2 & 10 & 2.7 & $\infty$ & 0.66 & 0.93 \\
\hline $\mathrm{Si}(422)$ & $\mathrm{Si}(400)$ & 35.2 & 7.3 & 1.9 & $\infty$ & 0.69 & 0.70 \\
\hline $\mathrm{Si}(511)$ & $\mathrm{Si}(400)$ & 15.8 & 10 & 2.2 & 0.5 & 0.5 & 0.75 \\
\hline
\end{tabular}

To confirm these results and to find the best design solution, phase space diagram simulations were used (described in section 2.4). The first take-off angle was fixed at $88^{\circ}$ (the basic shutter position). The monochromator location at HB-2 is sketched in Fig. 3.4. The distance $\mathrm{L}_{0}$ is $20 \mathrm{~cm}$ larger than in the single reflection option. Note that shutter rotation of $180^{\circ}$ around its horizontal axis is needed to accommodate this increase (i.e. opening is furthest away from source). Distances $L_{1}$, and $\mathrm{L}_{2}$ were chosen to maintain the same sample location on changing the wavelength. The distance between the axis of the beam produced by the first monochromator unit and the goniometer center was taken as $80 \mathrm{~cm}$. A multi-wafer unit made from [100] silicon wafers was assumed for the first crystal. A multi-wafer unit made from [110] wafers with the Si (331) reflection at $13.3^{\circ}$ cutting angle was also considered. The second unit was assumed to be of multi-wafer type made of [110] or [100] oriented wafers. Other design dimensions are listed below:

- distance from source to first unit:

$7.88 \mathrm{~m}$

- diameter of source:

$18 \mathrm{~cm}$

- opening of in-pile shutter:

$10 \times 10 \mathrm{~cm}$

- first unit dimensions:

$16.5 \times 15.2 \times 1.0 \mathrm{~cm}$

- opening of the reflected beam shutter:

$7.6 \times 12.7 \mathrm{~cm}$

- second unit dimensions:

$16.5 \times 15.2 \times 0.35 \mathrm{~cm}$

- distance between second unit and sample:

$80 \mathrm{~cm}$ 
- slits before and after sample:

$2 \times 2 \mathrm{~mm}$

- distance from sample to PSD:

$80 \mathrm{~cm}$

- PSD spatial resolution:

$2 \mathrm{~mm}$

The best results are summarized in Tables 3.5 and 3.6. Table 3.5 contains the following parameters:

- horizontal $(\Delta \gamma)$ and vertical $(\Delta \delta)$ angular beam divergences at sample position;

- peak reflectivity $P_{a 1}$ and $P_{a 2}$ for each unit;

- flux gain relative to (331) single reflection;

- FWHM at $2 \theta_{s}=90^{\circ}$.

Table 3.6 contains:

- cutting angles $\chi_{1}$ and $\chi_{2}$;

- optimum horizontal $\left(\mathrm{R}_{\mathrm{H} 1}, \mathbf{R}_{\mathrm{H} 2}\right)$ and vertical $\left(\mathrm{R}_{\mathrm{V} 1}, \mathrm{R}_{\mathrm{V} 2}\right)$ radii for $2 \theta_{s}=90^{\circ}$;

- distance $L_{1}$ between units and $L_{2}$ from the second crystal to goniometer center.

Table 3.5

\begin{tabular}{|c|c|c|c|c|c|c|c|c|}
\hline $\begin{array}{c}\text { Type } \\
1^{\text {st }} / 2^{\text {nd }}\end{array}$ & $\begin{array}{c}\lambda \\
{[\mathrm{nm}]}\end{array}$ & $\begin{array}{c}\theta_{2} \\
{[\mathrm{deg} .]}\end{array}$ & $\begin{array}{c}\Delta \gamma \\
{[\mathrm{deg} .]}\end{array}$ & $\begin{array}{c}\Delta \delta \\
{[\mathrm{deg} .]}\end{array}$ & $\mathrm{P}_{\mathrm{a} 1}$ & $\mathrm{P}_{\mathrm{a} 2}$ & Flux & $\begin{array}{c}\text { FWHM } \\
{[\mathrm{deg} .]}\end{array}$ \\
\hline $311 / 220$ & 0.227 & 72.66 & 4.7 & 9.0 & 0.39 & 0.68 & 5.0 & 0.28 \\
\hline $400 / 220$ & 0.189 & 58.84 & 3.7 & 8.1 & 0.65 & 0.61 & 3.0 & 0.20 \\
\hline $331 / 220$ & 0.173 & 53.59 & 2.4 & 7.6 & 0.53 & 0.58 & 2.5 & 0.24 \\
\hline $422 / 400$ & 0.154 & 69.11 & 5.5 & 8.8 & 0.30 & 0.35 & 7.1 & 0.32 \\
\hline $511 / 400$ & 0.145 & 64.65 & 4.1 & 8.5 & 0.27 & 0.33 & 2.4 & 0.23 \\
\hline
\end{tabular}

Table 3.6

\begin{tabular}{|c|c|c|c|c|c|c|c|c|}
\hline $\begin{array}{c}\text { Type } \\
1^{\text {st }} / 2^{\text {nd }}\end{array}$ & $\begin{array}{c}\chi_{1} \\
{[\mathrm{deg} .]}\end{array}$ & $\begin{array}{c}\chi_{2} \\
{[\mathrm{deg} .]}\end{array}$ & $\begin{array}{c}\mathrm{R}_{\mathrm{H} 1} \\
{[\mathrm{~m}]}\end{array}$ & $\begin{array}{c}\mathrm{R}_{\mathrm{H} 2} \\
{[\mathrm{~m}]}\end{array}$ & $\begin{array}{c}\mathrm{R}_{\mathrm{V} 1} \\
{[\mathrm{~m}]}\end{array}$ & $\begin{array}{c}\mathrm{R}_{\mathrm{V} 2} \\
{[\mathrm{~m}]}\end{array}$ & $\begin{array}{c}\mathrm{L}_{1} \\
{[\mathrm{~m}]}\end{array}$ & $\begin{array}{c}\mathrm{L}_{2} \\
{[\mathrm{~m}]}\end{array}$ \\
\hline $311 / 220$ & 25.4 & 0 & 5.7 & 2.24 & $\infty$ & 0.81 & 1.87 & 0.84 \\
\hline $400 / 220$ & 0 & 58.84 & 3.7 & 8.1 & $\infty$ & 0.81 & 1.64 & 0.94 \\
\hline $331 / 220$ & 13.3 & 53.59 & 2.4 & 7.6 & $\infty$ & 0.81 & 1.53 & 0.99 \\
\hline $422 / 400$ & 35.2 & 69.11 & 5.5 & 8.8 & $\infty$ & 0.81 & 1.82 & 0.86 \\
\hline $511 / 400$ & 15.8 & 64.65 & 4.1 & 8.5 & $\infty$ & 0.81 & 1.74 & 0.89 \\
\hline
\end{tabular}

The linewidth variation with detector angle $2 \theta_{\mathrm{s}}$ is shown in Fig. $3.5-3.8$. Similar to Figs. 2.20-2.23 for single reflection options, configurations are taken with 
either fixed radius or variable optimal radius. There is a remarkable difference between the single reflection arrangement compared with the double reflection arrangement. With single reflection it was possible to practically conserve linewidths in the whole range of scattering angles of interest by optimizing the horizontal curvature. Double reflection arrangements show a distinct worsening of linewidths at high scattering angles even at optimal radii (derived by numerical optimization of the figure of merit). The figure of merit with and without radius variation is shown in Fig. 3.9 - 3.10. Without radius variation (Fig. 3.9) the gain in figure of merit is high for only a limited range of sample d-spacings. With radius adjustment (Fig. 3.10) the gain in the figure of merit lies between 3 and 9 in the whole range of sample d-spacings of interest $(0.095-0.18 \mathrm{~nm})$.

\subsection{Recommended configuration}

A way to improve the performance of the double reflection monochromators is to consider adjustable wafer cutting angles, as discussed for single reflection configurations. For the second unit with [110] wafers symmetrical reflection is optimal. For the same unit with [100] wafers a small offset of 4 degrees seems to be beneficial.

The first unit with wafer orientation of about $12^{\circ}$ relative to [100] (the same choice as for single reflection) shows minor improvement of figures of merit for the (311) and (400) reflections, a small gain for the (511) reflection and a significant improvement for the (422) reflection only. More important seems to be the choice of crystal thickness. First crystal thickness must be large to accommodate a different phase space diagram inclination when the two crystals do not match. This occurs at detector angles larger that $90^{\circ}$ if one tries to reach focusing in scattering, because the relation (3.16) prescribes unphysical values for the first crystal curvature radius. A value of $2.4 \mathrm{~cm}$ for the first crystal thickness would be optimal, but would require big changes in the design mechanics with cost increase at unproven technical feasibility. For the second crystal five-wafer units are adequate in the $70-95^{\circ}$ detector angle range. At higher angles a single wafer is sufficient to obtain a better figure of merit. 
The basic performance and construction characteristics are given in Tables 3.7 and 3.8. Linewidths versus detector angle for this arrangement are given in Figs. 3.11 - 3.14. Figures of meril for fixed optimal radius at $2 \theta_{s}=90^{\circ}$ are shown in Fig. 3.15, and for variable optimal radius in Fig. 3.16. Gains in figure of merit from 6 to 12 are reached in the sample d-spacing range from $0.095 \mathrm{~nm}$ to $0.16 \mathrm{~nm}$. The gains are high even for samples with intrinsic micro-deformations because increased figures of merit come from increase of intensity, not from reduction of instrumental linewidths.

Table 3.7

\begin{tabular}{|c|c|c|c|c|c|c|c|c|}
\hline $\begin{array}{l}\text { Type } \\
1^{\text {st }} / 2^{\text {nd }}\end{array}$ & $\begin{array}{c}\lambda \\
{[\mathrm{nm}]}\end{array}$ & $\begin{array}{c}\theta_{2} \\
\text { [deg.] }\end{array}$ & $\begin{array}{c}\Delta \gamma \\
\text { [deg.] }\end{array}$ & $\begin{array}{c}\Delta \delta \\
\text { [deg.] }\end{array}$ & $P_{a 1}$ & $P_{a 2}$ & Flux & $\begin{array}{c}\text { FWHM } \\
\text { [deg.] }\end{array}$ \\
\hline $311 / 220$ & 0.227 & 72.66 & 4.3 & 9.0 & 0.56 & 0.68 & 6.8 & 0.31 \\
\hline $400 / 220$ & 0.189 & 58.84 & 4.0 & 8.1 & 0.95 & 0.61 & 5.8 & 0.21 \\
\hline $422 / 400$ & 0.154 & 69.11 & 5.3 & 8.8 & 0.40 & 0.37 & 8.4 & 0.29 \\
\hline $511 / 400$ & 0.145 & 64.65 & 4.8 & 8.5 & 0.38 & 0.34 & 3.8 & 0.23 \\
\hline
\end{tabular}

Table 3.8

\begin{tabular}{|c|c|c|c|c|c|c|c|c|}
\hline $\begin{array}{c}\text { Type } \\
1^{\text {st }} / 2^{\text {nd }}\end{array}$ & $\begin{array}{c}\chi_{1} \\
{[\text { deg.] }}\end{array}$ & $\begin{array}{c}\chi_{2} \\
{[\text { deg. }]}\end{array}$ & $\begin{array}{c}\mathrm{R}_{\mathrm{H} 1} \\
{[\mathrm{~m}]}\end{array}$ & $\begin{array}{c}\mathrm{R}_{\mathrm{H} 2} \\
{[\mathrm{~m}]}\end{array}$ & $\begin{array}{c}\mathrm{R}_{\mathrm{v} 1} \\
{[\mathrm{~m}]}\end{array}$ & $\begin{array}{c}\mathrm{R}_{\mathrm{V} 2} \\
{[\mathrm{~m}]}\end{array}$ & $\begin{array}{c}\mathrm{L}_{1} \\
{[\mathrm{~m}]}\end{array}$ & $\begin{array}{c}\mathrm{L}_{2} \\
{[\mathrm{~m}]}\end{array}$ \\
\hline $311 / 220$ & 13.2 & 0 & 5.1 & 2.24 & $\infty$ & 0.81 & 1.87 & 0.84 \\
\hline $400 / 220$ & -12 & 0 & 6.9 & 2.65 & $\infty$ & 0.81 & 1.64 & 0.94 \\
\hline $422 / 400$ & 23.2 & 4 & 9.1 & 2.57 & $\infty$ & 0.84 & 1.82 & 0.86 \\
\hline $511 / 400$ & 3.8 & 4 & 7.8 & 2.73 & $\infty$ & 0.84 & 1.74 & 0.89 \\
\hline
\end{tabular}

Double reflection options increase the divergence solid angle of the neutron beam falling onto the sample by a factor of 4 to 7 . Because of reflectivity losses the flux gain is less than 2 for the reflections (400), (331) and (511). A higher gain is possible with the (311) and (422) reflections. The (331) reflection, which needs a special unit in the single reflection option, can be replaced with a (422) reflection accessible in the same unit that gives the (400), (311) and (511) reflections.

The best choice for a double reflection monochromator (DRM) configuration at the new HB-2 neutron stress-mapping instrument would consist of three multi-wafer units with the following characteristics: 
1. First unit of DRM - used for (311), (400), (422) and (522) reflections:

- silicon wafer orientation: $12^{\circ}$ from [100] toward [011] direction;

- wafer thickness: $\sim 0.7 \mathrm{~mm}$;

- total crystal thickness: $>1.5 \mathrm{~cm}$;

- remotely adjustable radius of horizontal curvature from $1 \mathrm{~m}$ to $18 \mathrm{~m}$;

- flat in the vertical plane.

2. Second unit of DRM, option A - used for (220) reflection:

- silicon wafer orientation: [110];

- wafer thickness: $~ 0.7 \mathrm{~mm}$;

- wafer vertical segmentation : $5 \mathrm{~mm}$;

- total crystal thickness : $\sim 0.35 \mathrm{~cm}$;

- remotely adjustable radius of horizontal curvature from $1.5 \mathrm{~m}$ to $4 \mathrm{~m}$;

- fixed radius of vertical curvature: $0.84 \mathrm{~m}$

3. Second unit of DRM, option B - used for (400) reflection:

- silicon wafer orientation: $4^{\circ}$ from [100] toward [011] direction;

- wafer thickness: $\sim 0.7 \mathrm{~mm}$;

- wafer vertical segmentation : 5 mm;

- total crystal thickness: $\sim 0.35 \mathrm{~cm}$;

- remotely adjustable radius of horizontal curvature from $1.5 \mathrm{~m}$ to $4 \mathrm{~m}$;

- fixed vertical radius: $\quad 0.81 \mathrm{~m}$

The first unit will be placed inside the main shield. The second crystal units can be mounted on the same $X Y \psi$ monochromator stage. The whole stage must have the possibility to be translated about $35 \mathrm{~cm}$ along the beam reflected by the first crystal to maintain the same sample location on changing the wavelength.

To improve the figure of merit of the double reflection monochromator (DRM) in the detector angle range $100^{\circ}-110^{\circ}$ for samples without microstrain, the second unit options can be replaced with one-wafer units (options $\mathrm{C}$ and D) of the same kind as options A and B. An additional gain with a factor of about 2 can be also reached by changing the silicon wafers with germanium wafers in the second unit, option B. 


\section{CONCLUSIONS}

1. For a beam cross section of $16.5 \times 15.2 \mathrm{~cm}$ at the monochromator location within the new HB-2 shielding, the maximum deflection angle of the beam axis (relative to the beamport axis) is approximately $2^{\circ}$. The maximum angular acceptance at this monochromator position is approximately $1^{0}$ in both the horizontal and the vertical planes. A primary beam shutter opening of $80-100 \mathrm{~cm}^{2}$ (width and height of 9 to $10 \mathrm{~cm}$ ) gives an effective transmission for useful neutrons better than $85 \%$ ( $60-70 \%$ overall neutron transmission) in all considered configurations. Reducing the opening to $7 \times 7 \mathrm{~cm}$ decreases the effective transmission to about $60 \%$ (40\% overall neutron transmission). An opening in the monochromator shutter $7.6 \mathrm{~cm}$ wide and $12.7 \mathrm{~cm}$ high is adequate for an efficient extraction of focused beams for residual stress studies. For the single reflection option a trapezoidal coarse collimator can be placed inside the monochromator shutter opening to reduce the background. A fixed take-off angle of the monochromator of $2 \theta_{M}=88^{\circ}$ is recommended. A variable take-off angle would give only modest gains in the figure of merit, which would not justify the cost and would delay the construction of a special rotating shutter in the wall of the new HB-2 main shield. Better gains are obtained by adjusting the horizontal curvature for each specimen of interest of $\mathrm{d}$-spacing between 0.09 and $0.19 \mathrm{~nm}$.

2. For the single reflection monochromator (SRM) option, two multi-wafer focusing units are adequate to cover a range of $0.09-0.19 \mathrm{~nm}$ in d-spacings at $2 \theta_{\mathrm{M}}=88^{\circ}$. Both units must have a remotely adjustable radius of horizontal curvature. A fixed vertical curvature is adequate. Both units should have 18 wafer packets $(12.5 \mathrm{~mm}$ thick). One unit (SRM option A) would use silicon wafers specially cut with $12^{\circ}$ offset from the [100] toward the [011] direction. The basic silicon reflections for this unit would be (311), (400) and (511), with wavelengths of $0.227,0.189$, and $0.145 \mathrm{~nm}$, respectively. The other unit (SRM option B) would use silicon wafers of [110] orientation. The basic reflection would be (331) with a wavelength of 0.173 $n m$. These two units can be mounted on the same remotely controlled XY $\psi$ monochromator stage. 
3. Successive Bragg reflections on two matching bent crystals in $(+,+)$ configuration can give significant increases of neutron flux at the sample by strong spatial focusing. The only solution within the restrictions of HB-2 is a double reflection configuration with the second crystal placed outside the main shield. A different position of the secondary shutter (rotation with $180^{\circ}$ around its horizontal axis) is necessary to accommodate the diffractometer platform placement. For the double reflection monochromator (DRM) option the first multi-wafer unit would be placed inside the main shield. This unit would deliver the following wavelengths: $0.227,0.189,0.154$ and 0.145 corresponding to (311), (400), (422) and (511) respectively. The same orientations of the wafers as for SRM option A will be adequate, but in the first unit of double reflection monochromators the wafers should be vertically flat. A larger total crystal thickness is desirable. The units outside the main shield (second unit DRM option A and B) will have wafers of [100] and [110] orientation and packets of 5 wafers only. These two units can be mounted on the same remotely controlled XY $\Psi$ monochromator stage, as in the case of single reflection options. The stage has to be translated $(-35 \mathrm{~cm})$ along the reflected beam from the first unit in order to deliver the secondary reflected beam to the center of the sample goniometer.

4. Four optimal monochromator configurations, with an increasing degree of complexity, can be considered in conclusion. All make use of multi-wafer monochromator units with different specifications. The principal features and performances are summarized in Table 4.1.

Research sponsored by the Assistant Secretary for Energy Efficiency and Renewable Energy, Office of Transportation Technologies, as part of the High Temperature Materials Laboratory User Program, Oak Ridge National Laboratory, managed by Lockheed Martin Energy Research Corp. for the U.S. Department of Energy under contract number DE-AC05-96OR22464. 
Table 4.1

\begin{tabular}{|c|c|c|c|c|c|}
\hline Option & Material & Reflection & $\begin{array}{c}\text { Figure of } \\
\text { merit }^{*}\end{array}$ & $\begin{array}{l}\text { Average } \\
\text { gain }\end{array}$ & $\begin{array}{c}\text { New } \\
\text { design }\end{array}$ \\
\hline \multirow{3}{*}{$\begin{array}{c}\text { A } \\
\text { Single } \\
\text { One unit }\end{array}$} & \multirow[t]{3}{*}{ Si(100) $1 \mathrm{~cm}$ thick, commercial } & (311) & $0.8 / 0.5 / 0.2$ & \multirow[t]{3}{*}{-} & \multirow[t]{3}{*}{ No } \\
\hline & & (400) & $4.0 / 2.1 / 0.9$ & & \\
\hline & & $(511)$ & $2.7 / 1.5 / 0.7$ & & \\
\hline \multirow{4}{*}{$\begin{array}{c}\text { B } \\
\text { Single } \\
\text { Two units }\end{array}$} & \multirow[t]{3}{*}{$\mathrm{Si}(100) 1.2 \mathrm{~cm}$ thick, special cut } & $(311)$ & $2.1 / 1.3 / 0.6$ & \multirow{4}{*}{$\begin{array}{c}2.0 \\
\text { relative } \\
\text { to } \mathrm{A}\end{array}$} & \multirow[t]{4}{*}{ Yes } \\
\hline & & $(400)$ & $5.7 / 2.9 / 1.2$ & & \\
\hline & & (511) & $3.4 / 2.0 / 0.9$ & & \\
\hline & Si(110) $1.2 \mathrm{~cm}$ thick, special cut & $(331)$ & $4.5 / 2.3 / 0.9$ & & \\
\hline \multirow{4}{*}{$\begin{array}{c}\text { C } \\
\text { Double } \\
3 \text { units }\end{array}$} & \multirow{4}{*}{$\begin{array}{l}\text { Si(100) } 2.4 \mathrm{~cm} \text { thick, special cut } \\
\mathrm{Si}(110) 3.5 \mathrm{~mm} \text { thick, special cut } \\
\mathrm{Si}(100) 3.5 \mathrm{~mm} \text { thick, } \\
\text { commercial }\end{array}$} & $(311) /(220)$ & $6.8 / 3.9 / 1.8$ & \multirow{4}{*}{$\begin{array}{c}2.4 \\
\text { relative } \\
\text { to } B\end{array}$} & \multirow[t]{4}{*}{ Yes } \\
\hline & & $(400) /(220)$ & $7.3 / 4.1 / 1.8$ & & \\
\hline & & $(422) /(400)$ & $6.3 / 3.6 / 1.8$ & & \\
\hline & & $(511) /(400)$ & $3.2 / 1.7 / 0.8$ & & \\
\hline $\begin{array}{c}\mathrm{D} \\
\text { Double } \\
3 \text { units } \\
\end{array}$ & $\begin{array}{l}\text { Si(100) } 2.4 \mathrm{~cm} \text { thick, special cut } \\
\text { Si(110) } 3.5 \mathrm{~mm} \text { thick, special cut } \\
\text { Ge(100) } 3.5 \mathrm{~mm} \text { thick, } \\
\text { commercial }\end{array}$ & As C & $\begin{array}{c}\times 2 \text { for } \\
(422) /(400) \\
\text { and } \\
(511) /(400)\end{array}$ & $\begin{array}{c}3.1 \\
\text { relative } \\
\text { to } B\end{array}$ & Yes \\
\hline
\end{tabular}

* The average relative figure of merit for three values of the microstrain $(0,0.002$, 0.004) are given. The figure of merit of the present Si (331) bulk crystal unit on measuring the (211) line of $\alpha$-iron without microstrain was taken as reference (figure of merit $=1$ ). The averaging $d$-spacing range was $0.09-0.19 \mathrm{~nm}$.

* The average was performed considering the best monochromator choice for every dspacing in the range $0.09-0.19 \mathrm{~nm}$ and all values corresponding for three values of the microstrain $(0,0.002,0.004)$. 


\section{REFERENCES}

1. A. K. Freund and J. B. Forsyth, in Neutron Scattering in Materials Science, edited by G. Kostorz, Vol. 15 of A Treatise of Materials Sci. and Techn., 462-511 (1979).

2. A.W. Hewat, Mat. Sci. Forum, 9, 69-80 (1986).

3. J.D. Axe, S. Cheung, D.E. Cox, L. Passell, T. Vogt and S. Bar-Ziv, J. Neutron Research, 2(3), 85-94 (1994).

4. M. Popovici, W.B. Yelon, J. Neutron Research, 3(1), 1-25 (95); J. Neutron Research, 5, 227-239 (1997).

5. A.D. Stoica and M. Popovici, J. Appl. Cryst., 22,448-454 (1989).

6. W.B. Yelon, R. Berliner and M. Popovici, Physica B, 241-243, 237-239 (1998).

7. R.H. Scherm and E. Kruger, Nucl. Instr. Meth, A 338, 1-8 (1994).

8. M. Popovici and A.D. Stoica, J. Phys. E: Sci. Instr., 16, 662-665 (1983).

9. M. Popovici, W.B. Yelon, R. Berliner, A. D. Stoica and R. Law, Nucl. Instr. Meth., A 338, 99-110 (1994).

10. M. Popovici, A.D. Stoica and I. Ionita, J. Appl. Cryst., 20, 90-101 (1987).

11. M. Popovici, A.D. Stoica, B. Chalupa and P. Mikula, J. Appl. Cryst., 21, 258-265 (1988).

12. H. Maier-Leibnitz, Proc. Symp. Neutron Inelastic Scattering, Grenoble, 1972, Vienna: IAEA, p.681-696 (1972).

13. A.D. Stoica, Acta Cryst., A31, 189-193 (1975).

14. M.J. Cooper and R. Nathans, Acta Cryst., 23, 357 (1967).

15. T. J. Davis, X-Ray Sci. Tech., 1, 180 (1990).

16. M. Popovici and W.B. Yelon, Nucl. Instr. Meth., A316, 141-148 (1992).

17. M. Popovici and W.B. Yelon, SPIE Proc. Series, Vol.1738, $422-430$ (1992); Nucl. Instr. Meth., A338, 132-135 (1994); Zeitschrift fur Kristallografie, 209, 640-648 (1994). 


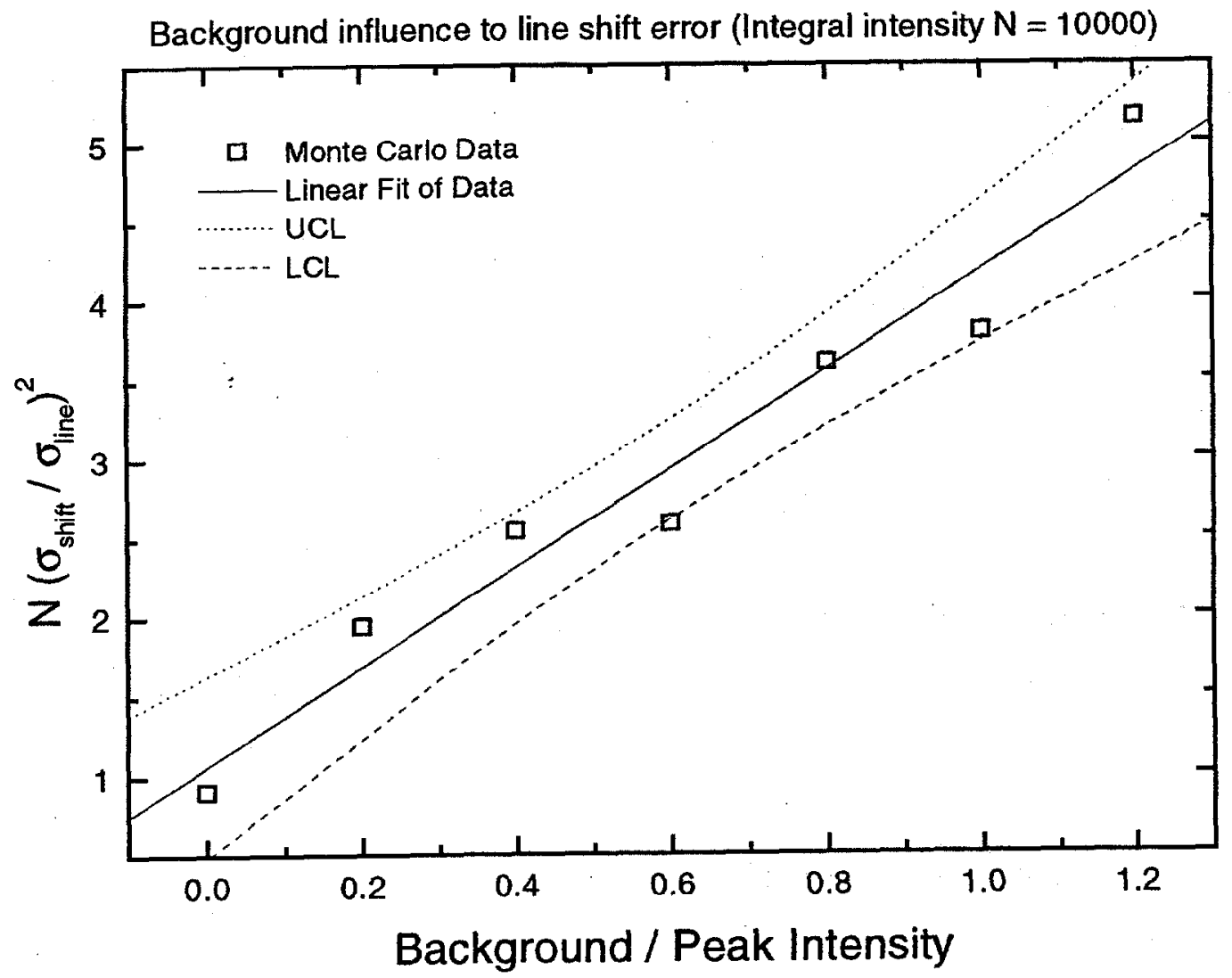

Fig. 1.1 Monte Carlo simulation of peak position accuracy with a constant background. 

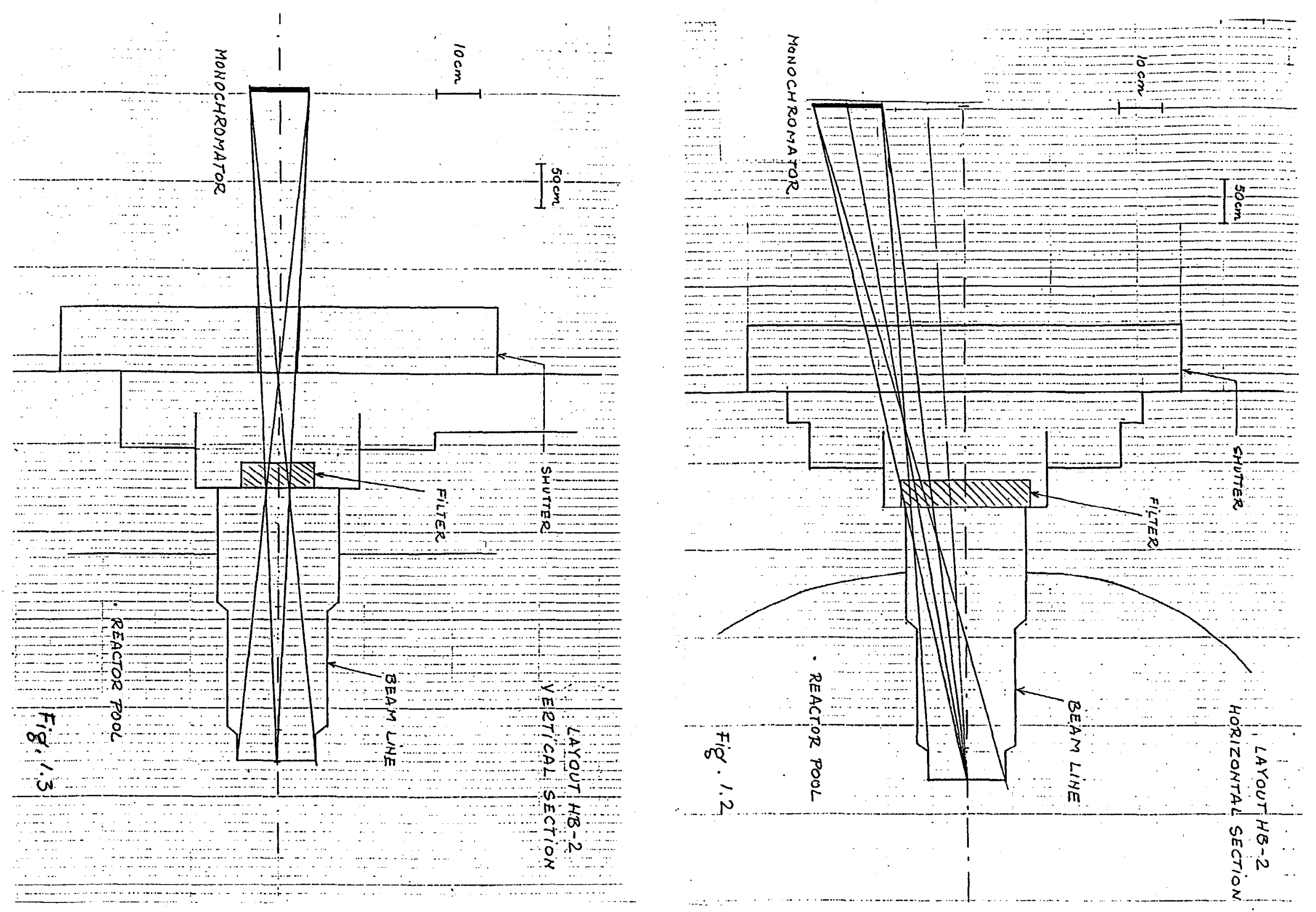


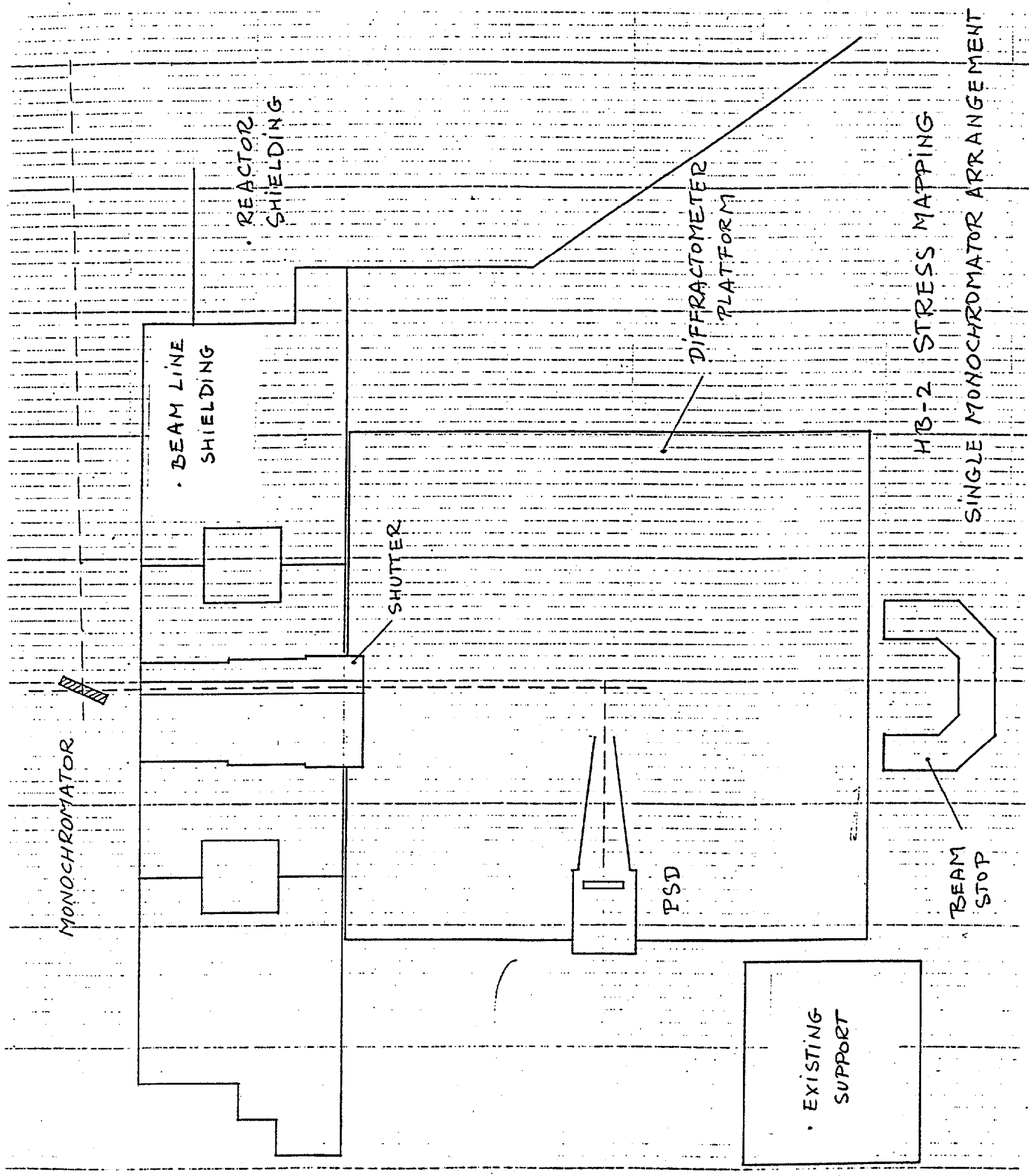

$\frac{1}{i}$ 


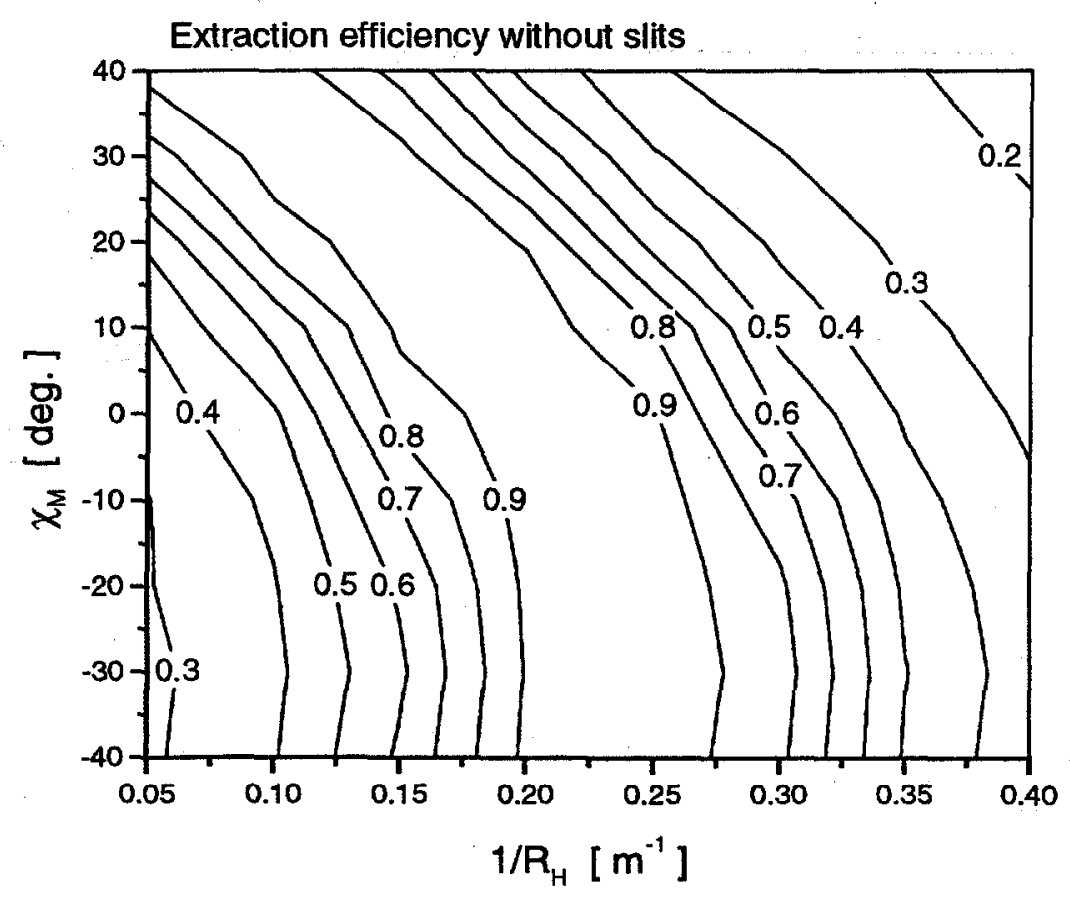

Fig.1.5. Extraction efficiency map - size of the source $(18 \mathrm{~cm}$ diameter) taking into account.

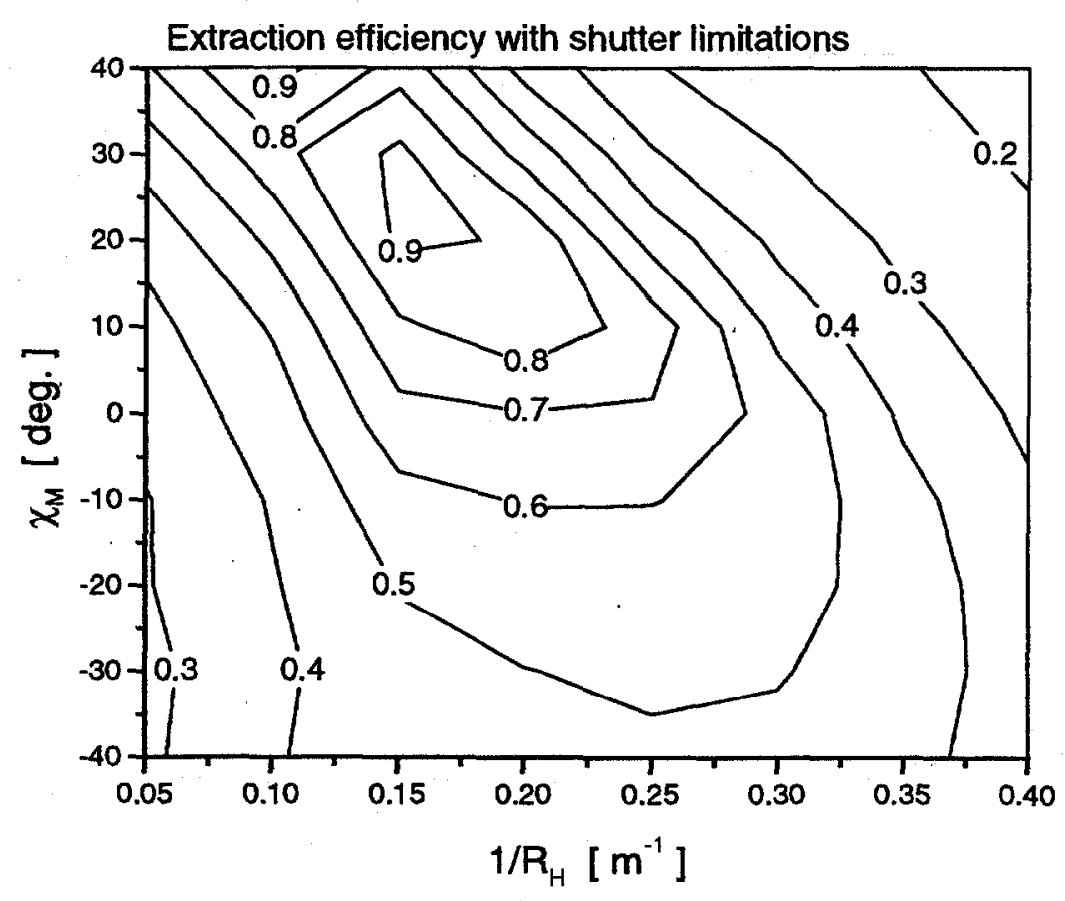

Fig.1.6. Extraction efficiency map - primary beam shutter opening: $10 \times 10 \mathrm{~cm}$; experiment shutter opening: $7.6 \times 12.7 \mathrm{~cm}$. 


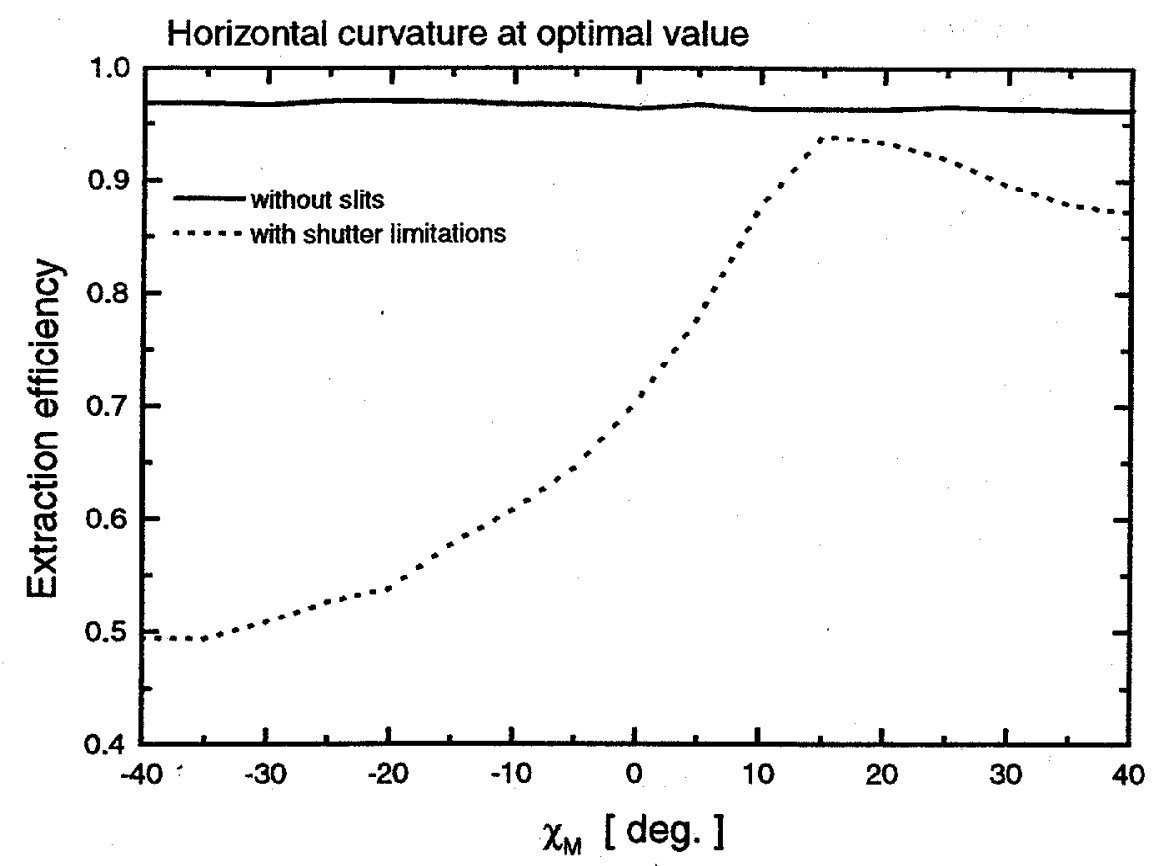

Fig.1.7. Extraction efficiency versus cutting angle - primary beam shutter opening: $10 \times$ $10 \mathrm{~cm}$; experiment shutter opening: $7.6 \times 12.7 \mathrm{~cm}$.

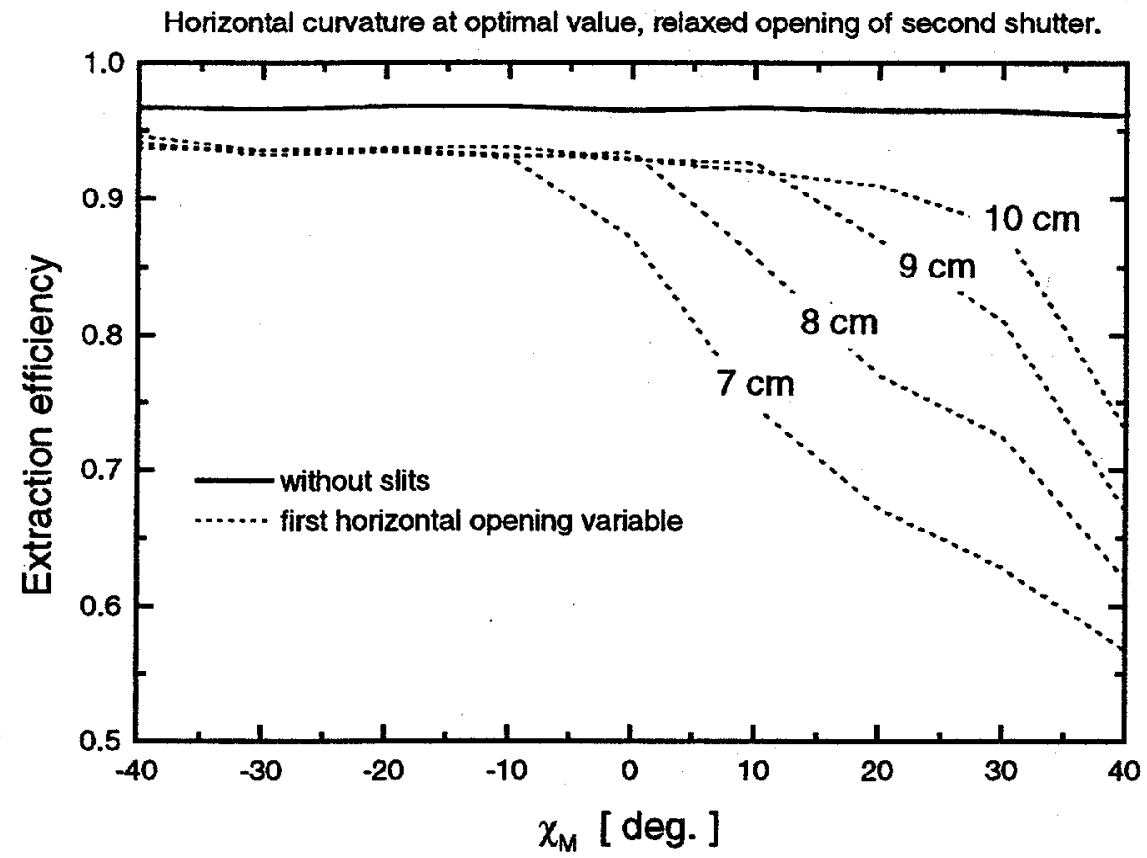

Fig.1.8. Extraction efficiency versus cutting angle - primary beam shutter horizontal opening dependence. 


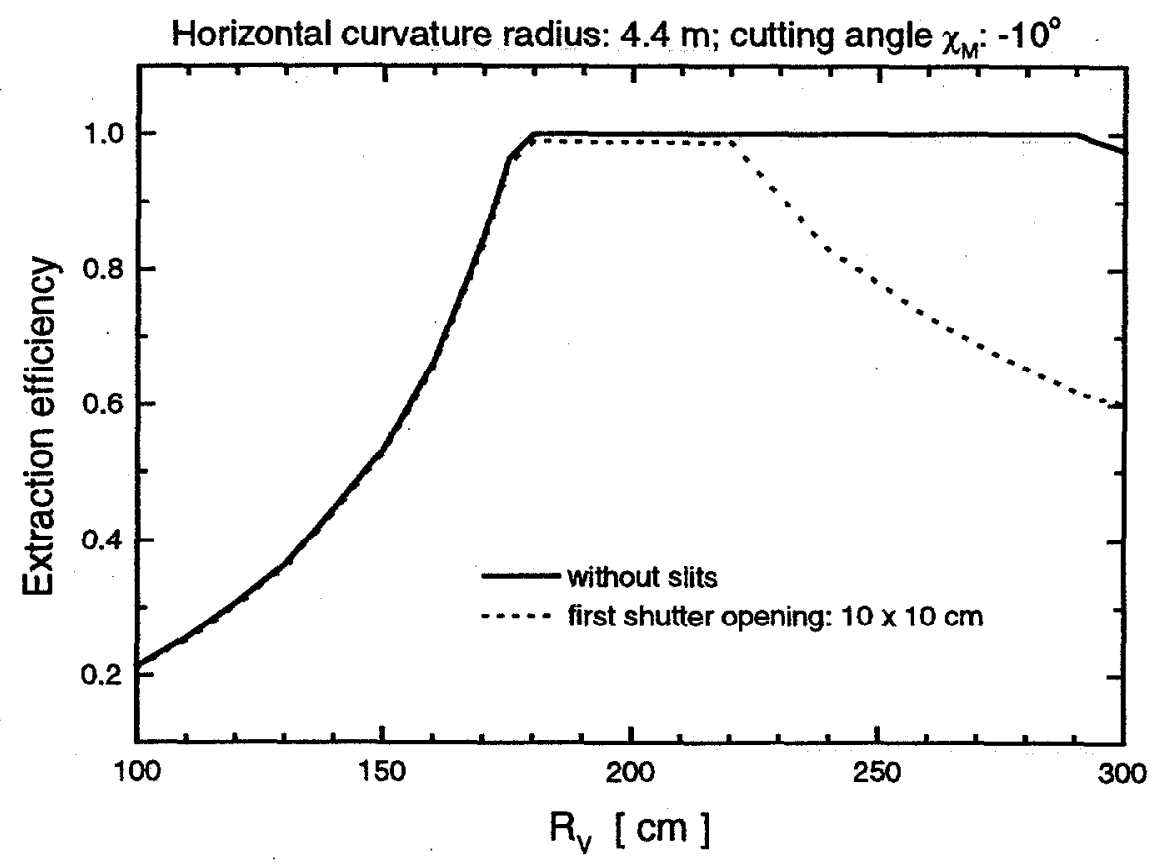

Fig.1.9. Extraction efficiency versus monochromator vertical curvature radius - without limitations in the secondary beam. 


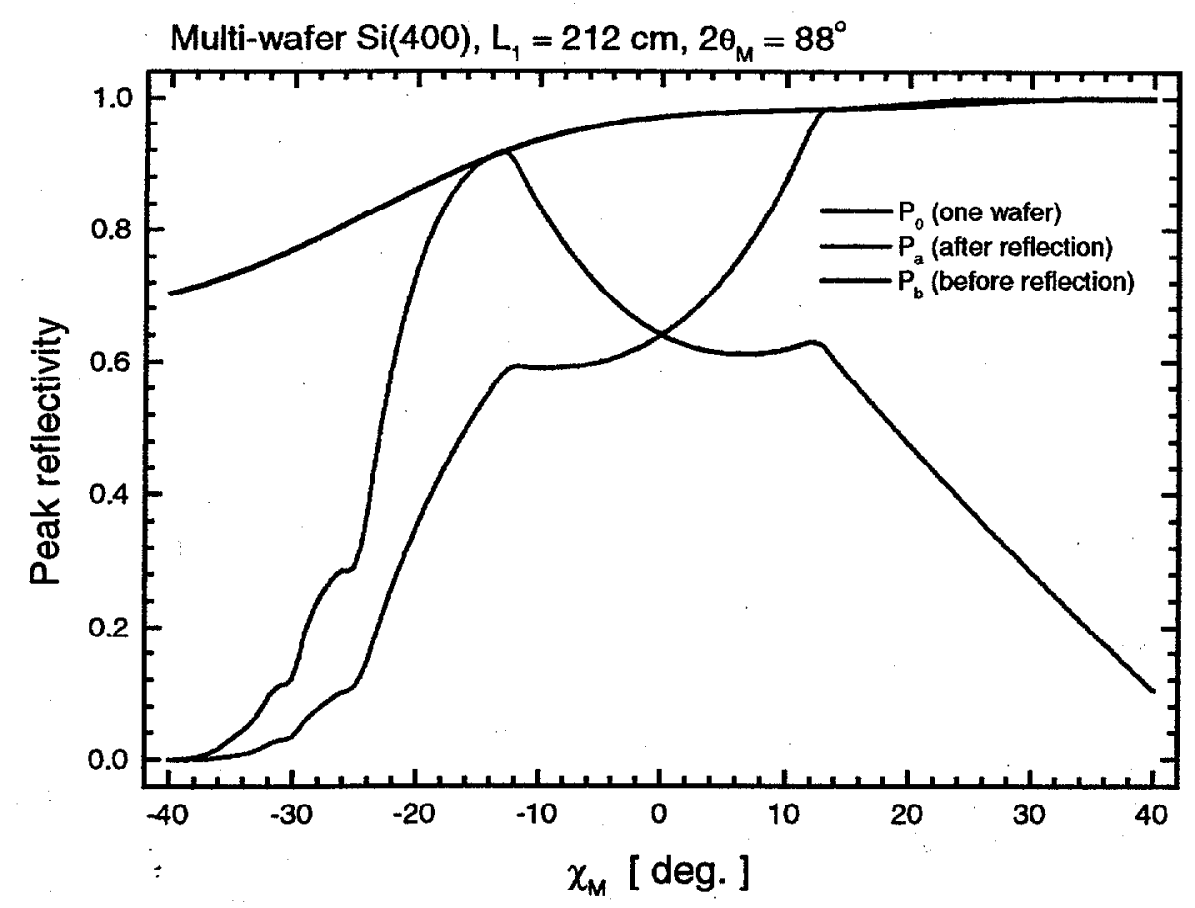

Fig. 2.1

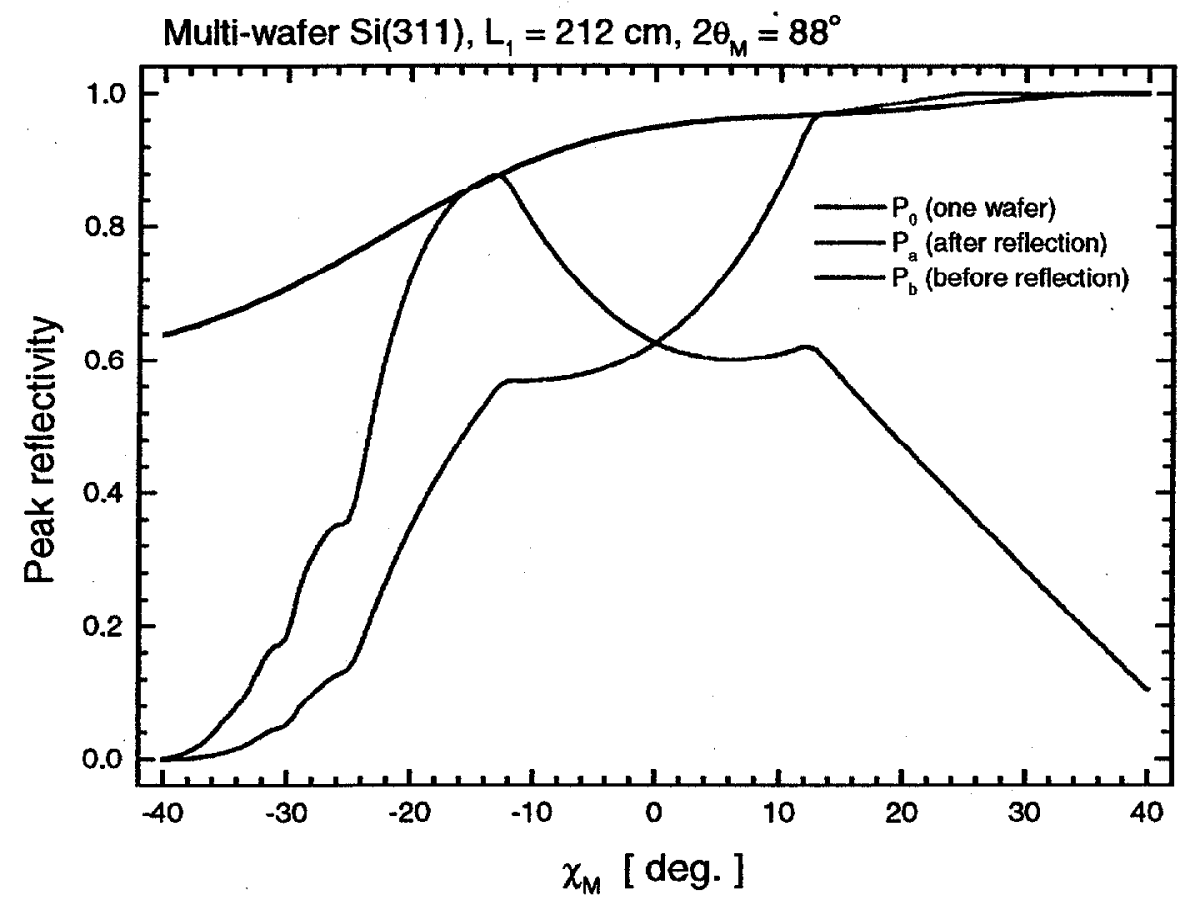

Fig. 2.2 


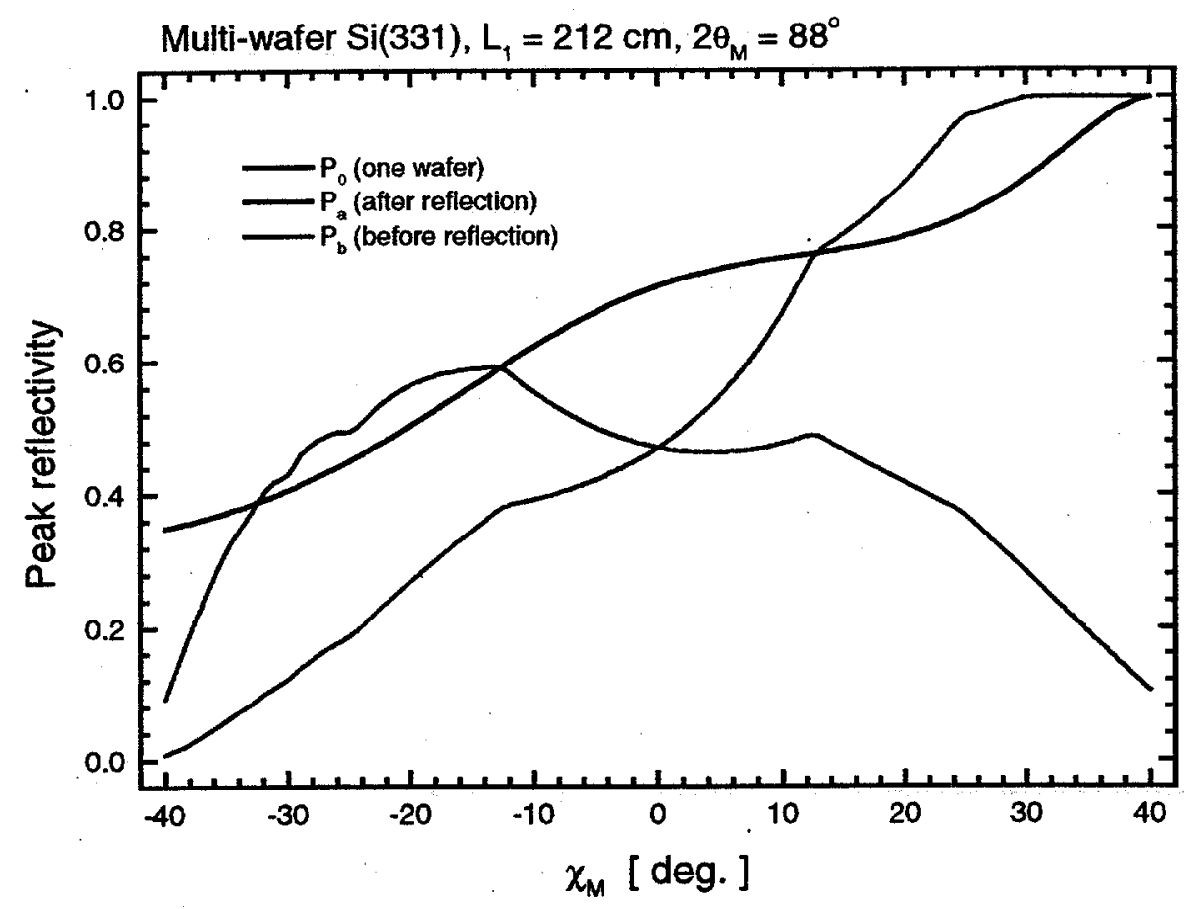

Fig. 2.3

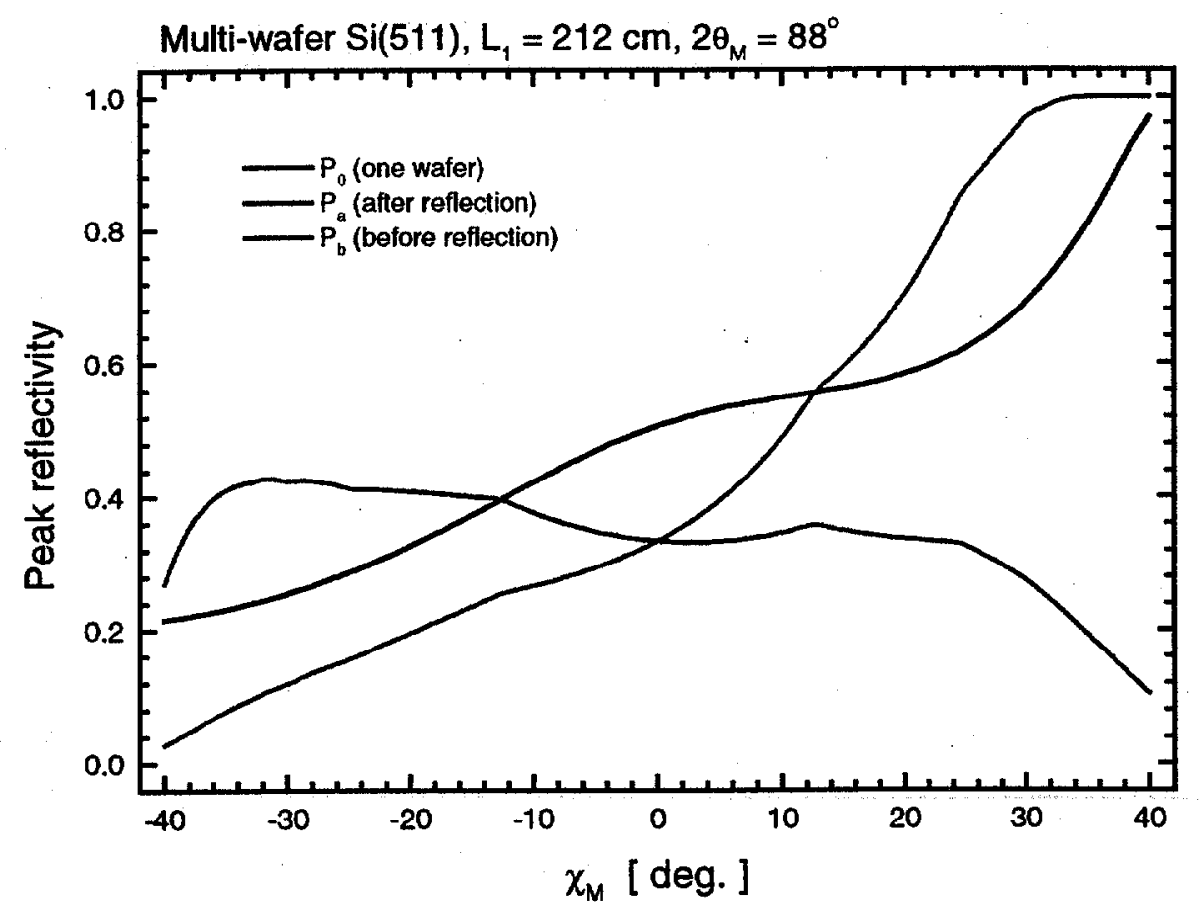

Fig. 2.4 


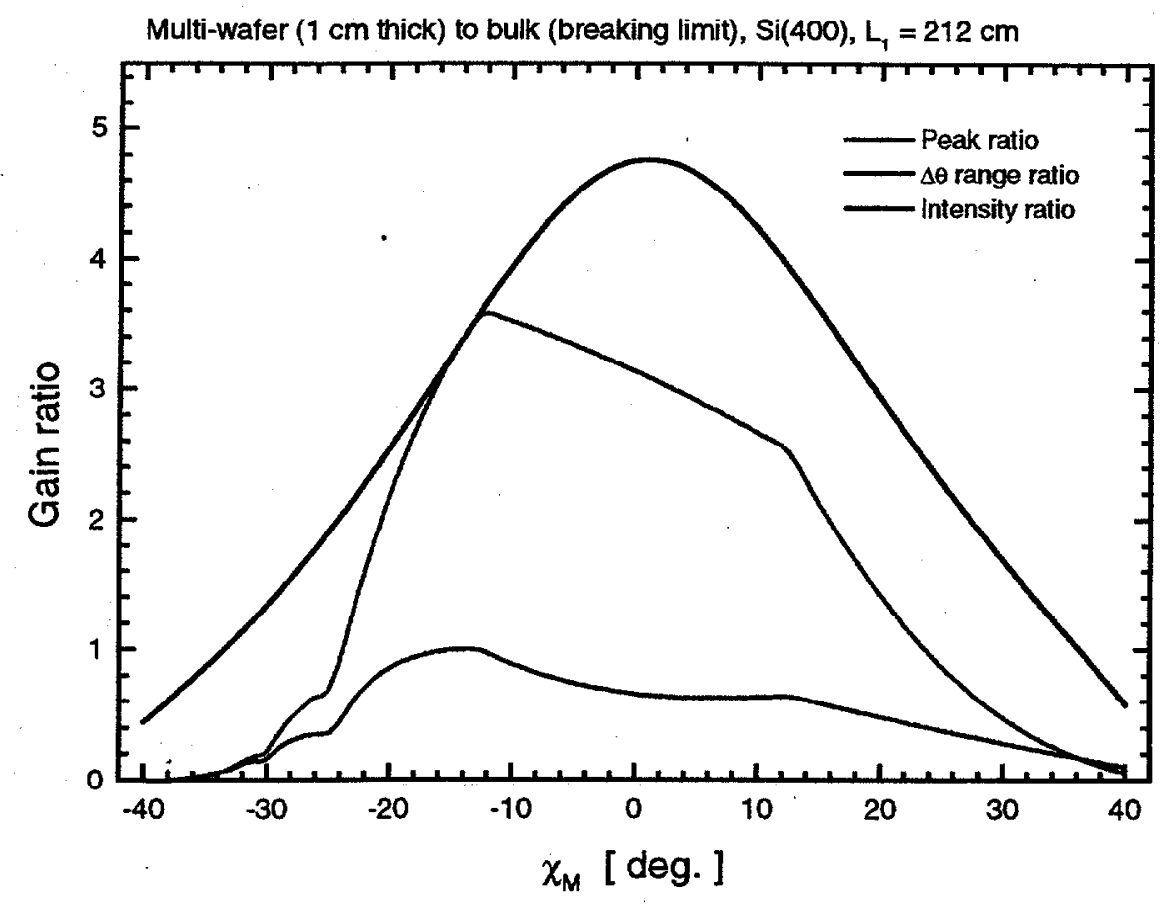

Fig. 2.5

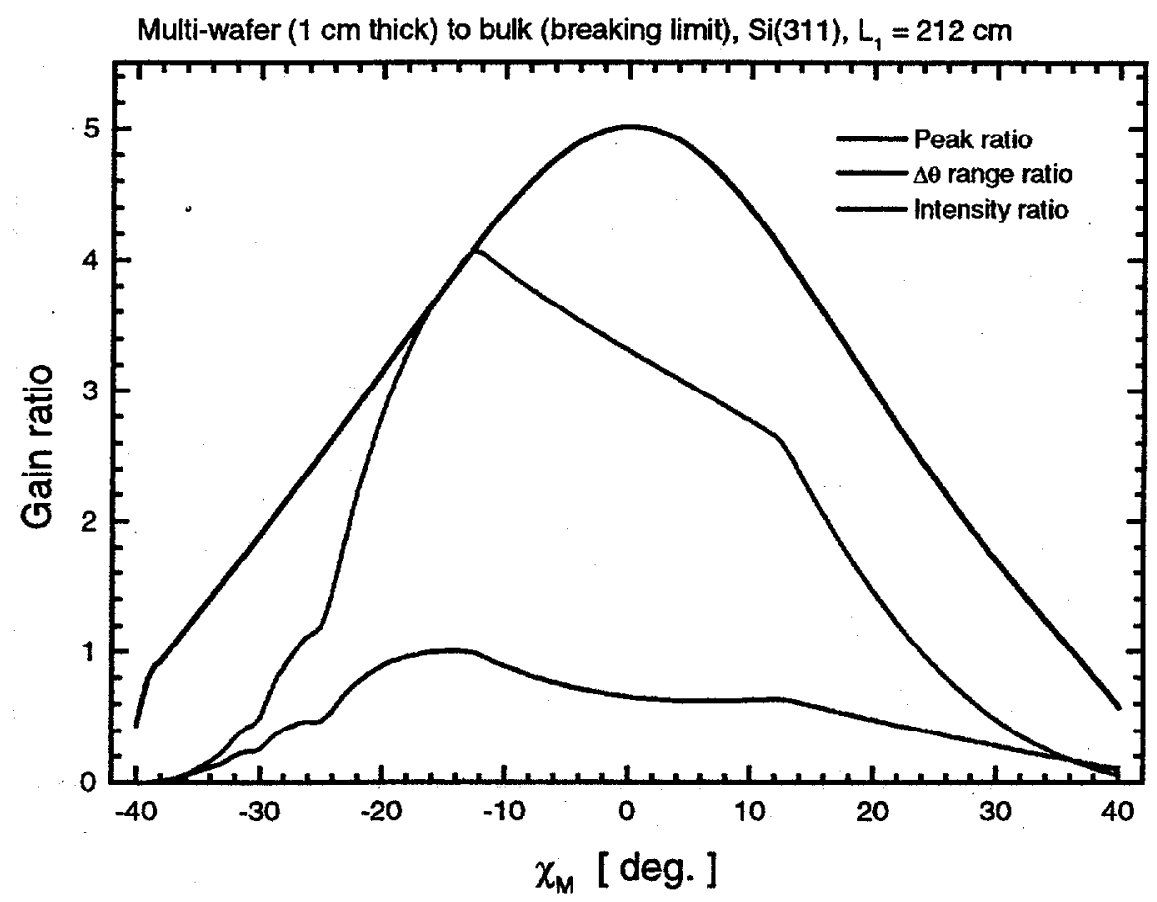

Fig. 2.6 


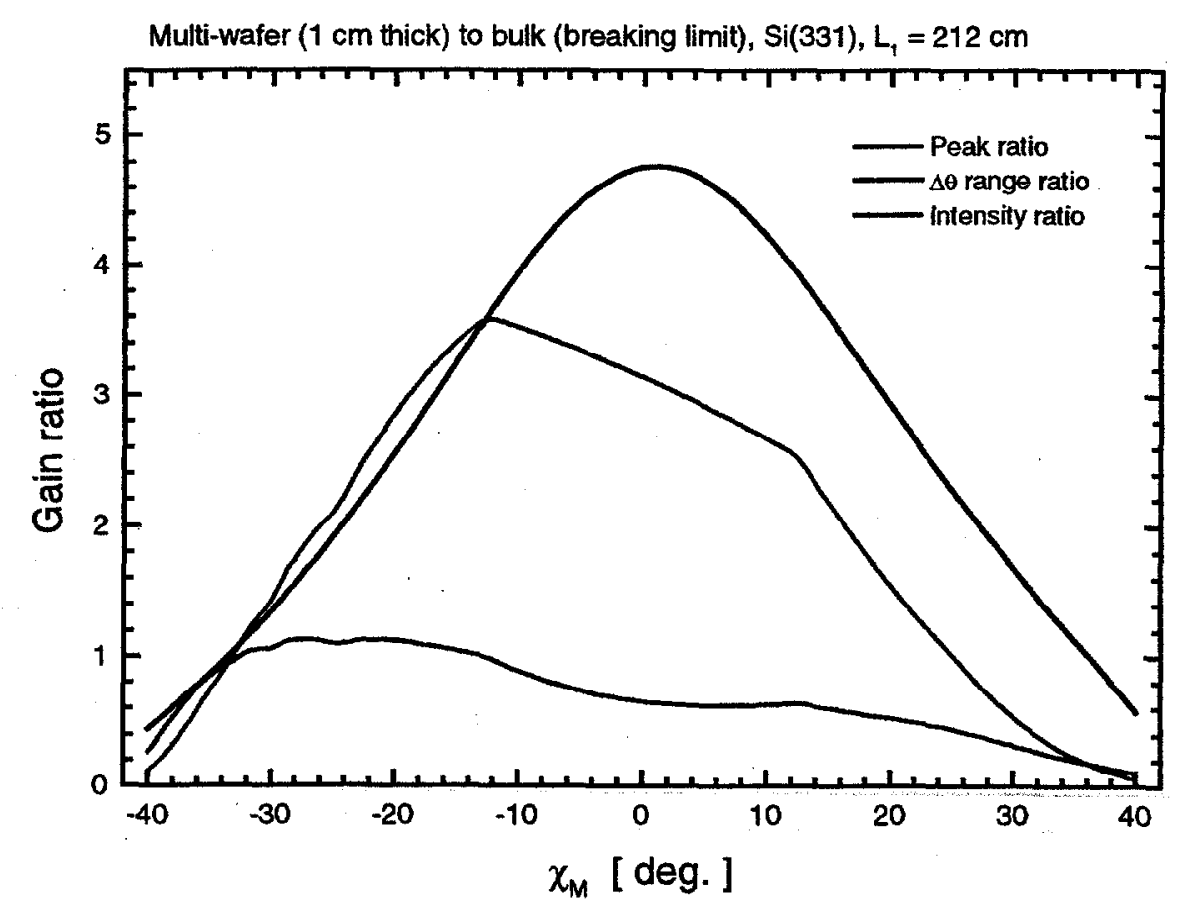

Fig. 2.7

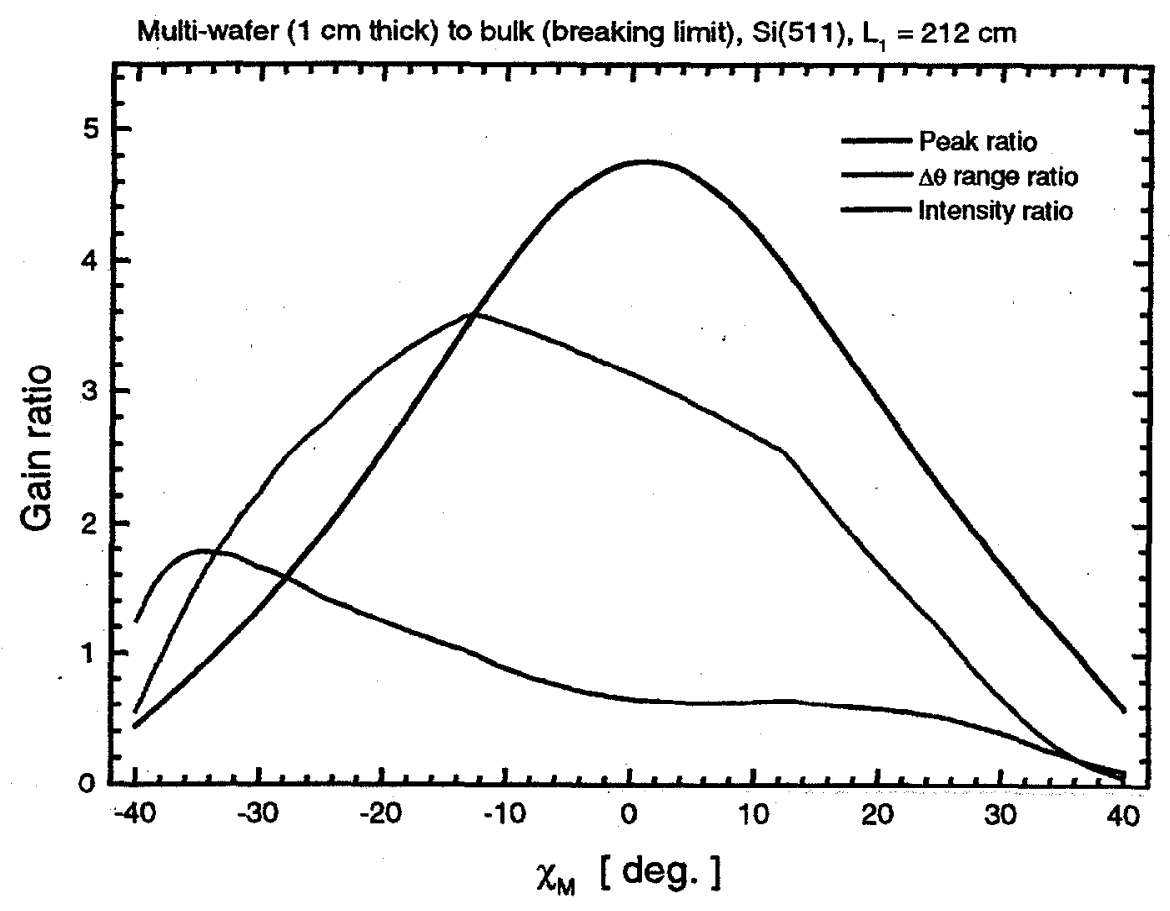

Fig. 2.8 


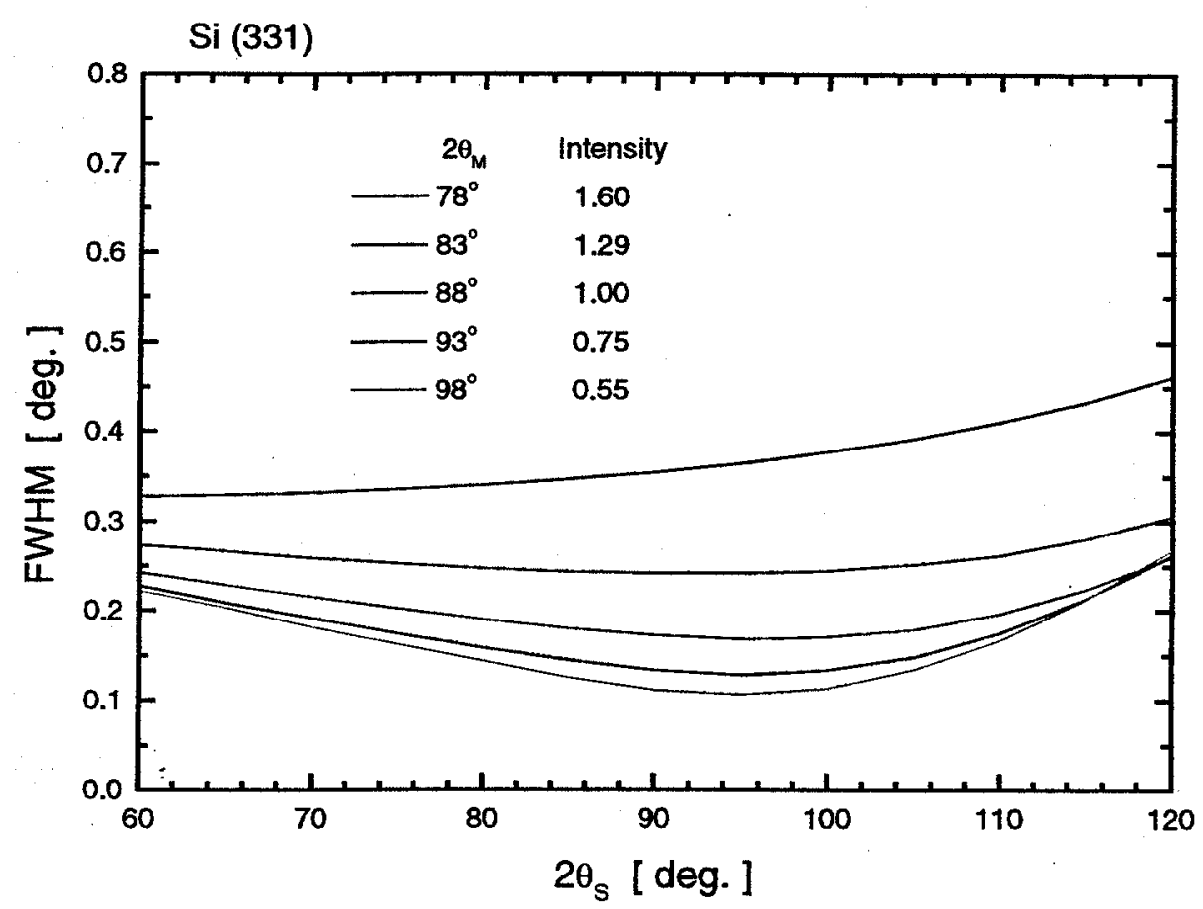

Fig. 2.9

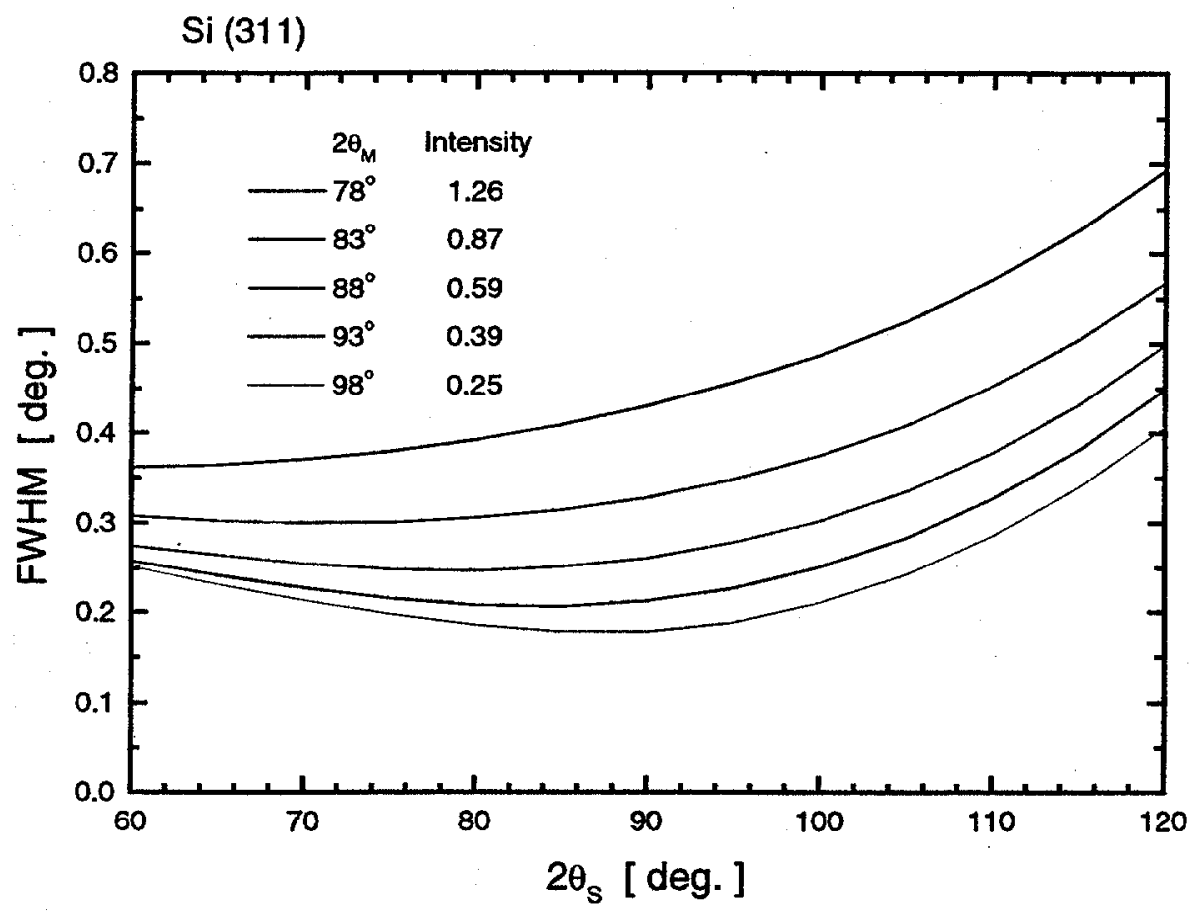

Fig. 2.10 


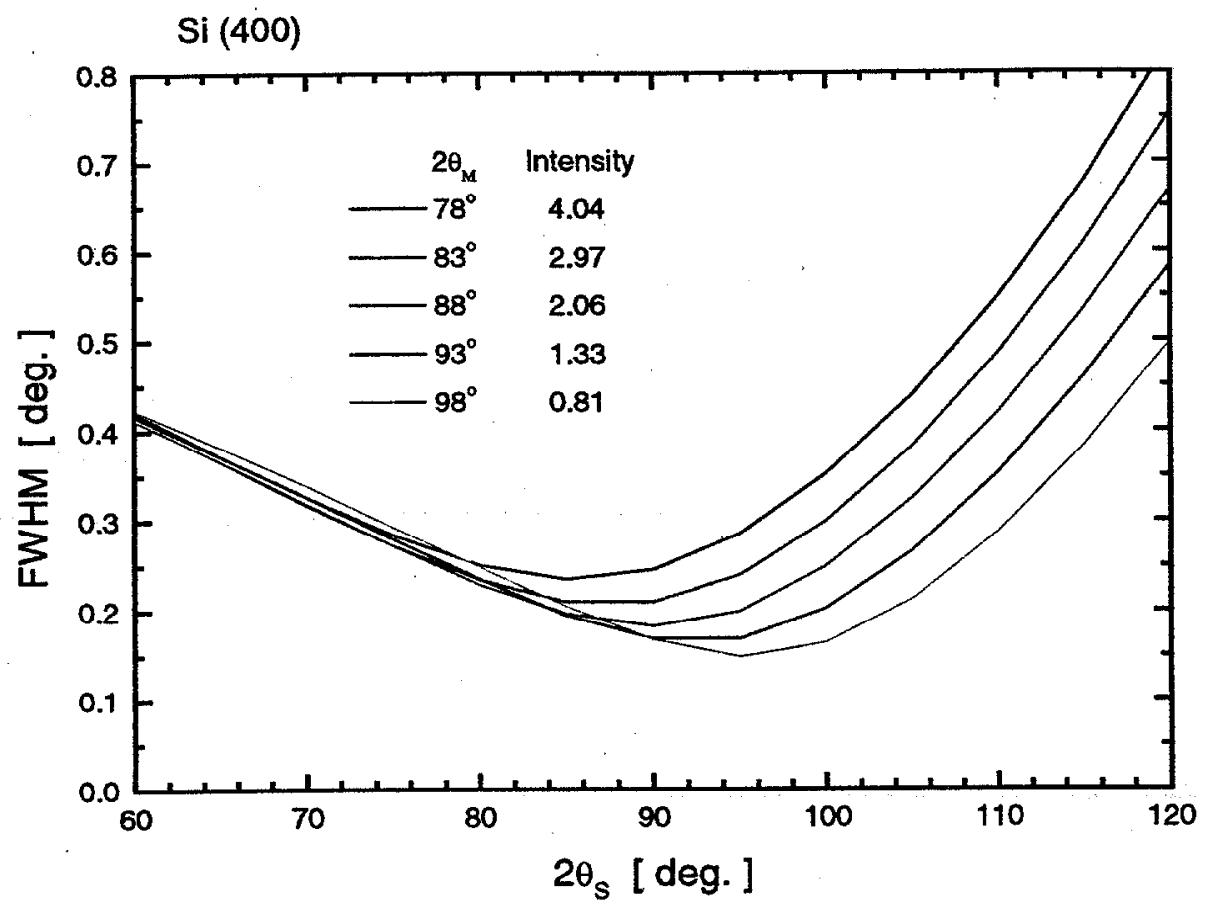

Fig. 2.11

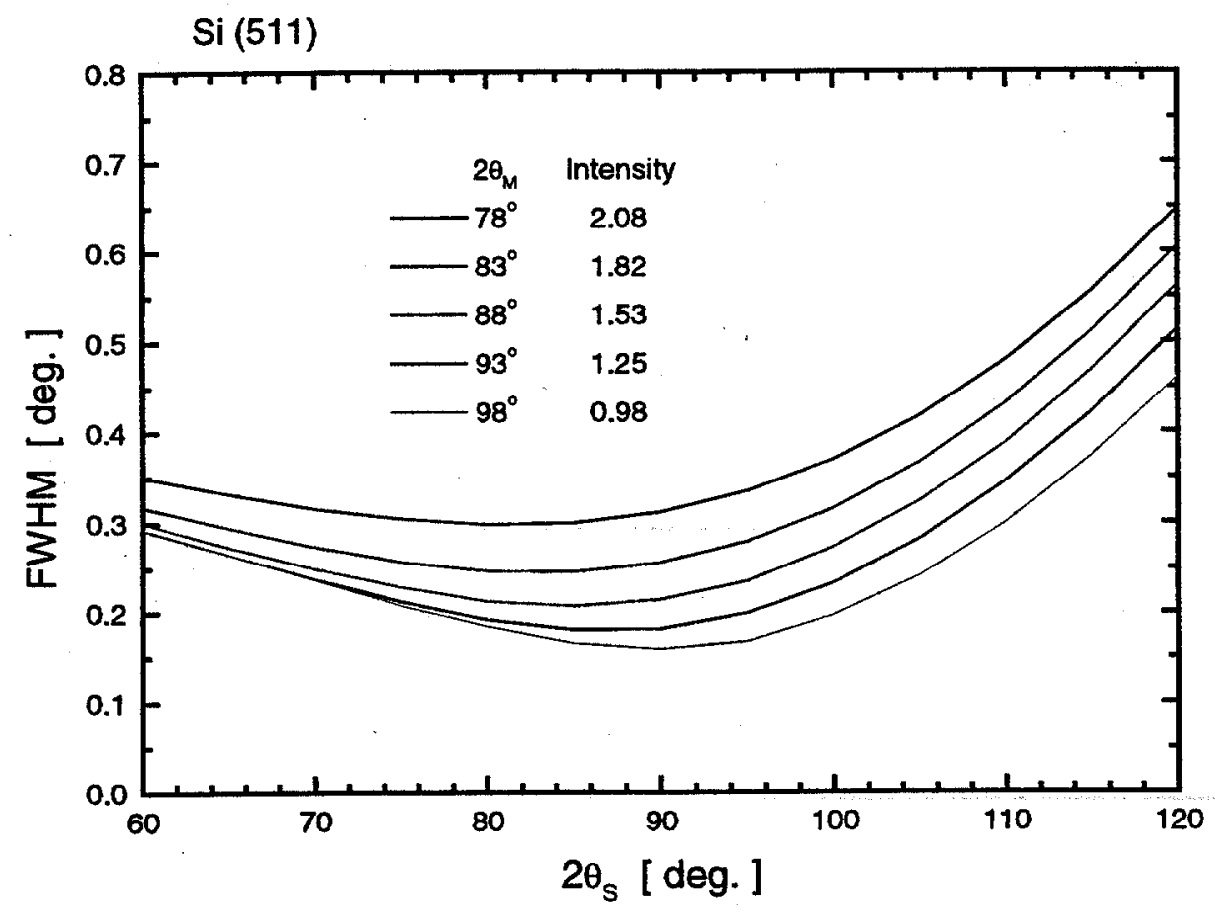

Fig. 2.12 


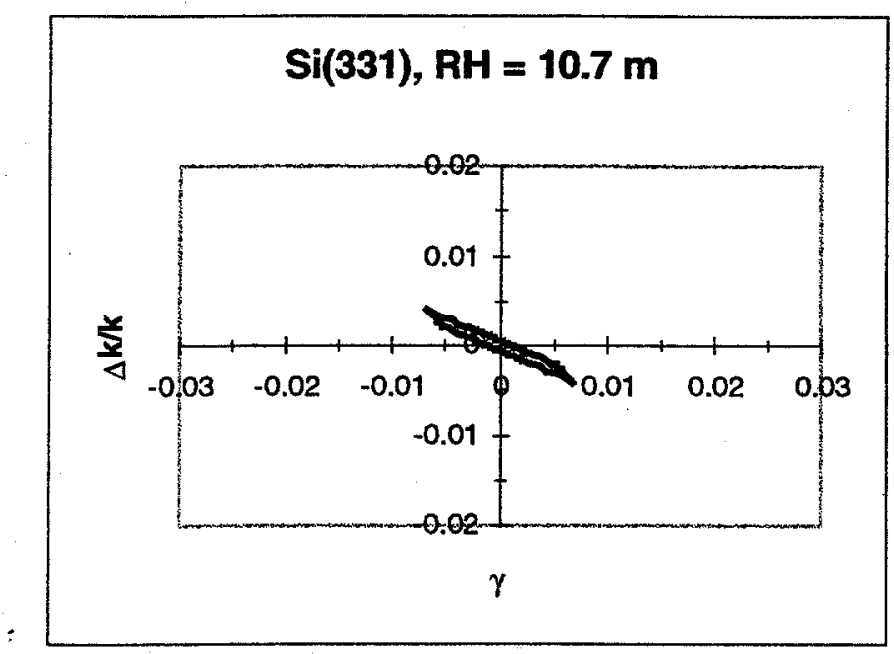

Fig. 2.13

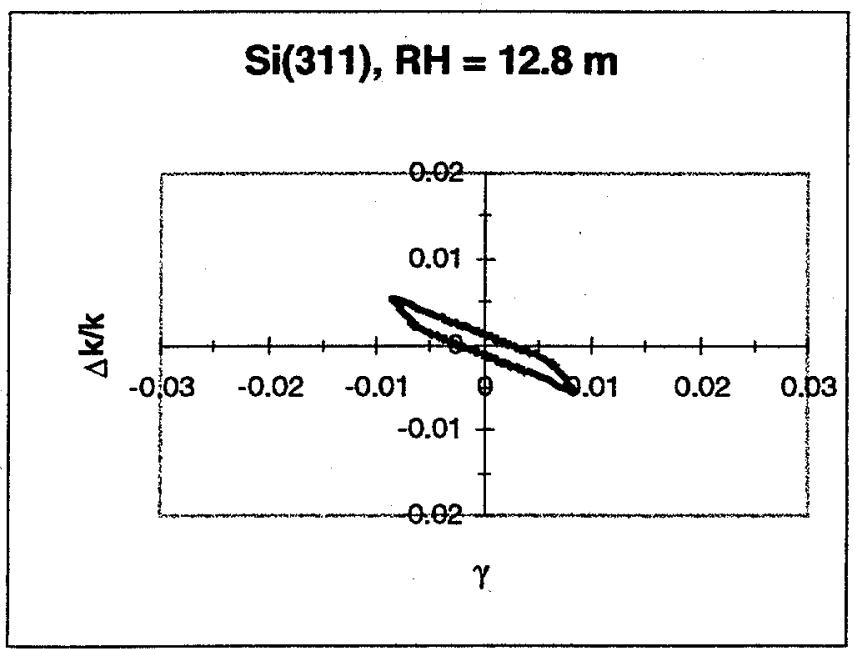

Fig. 2.14 


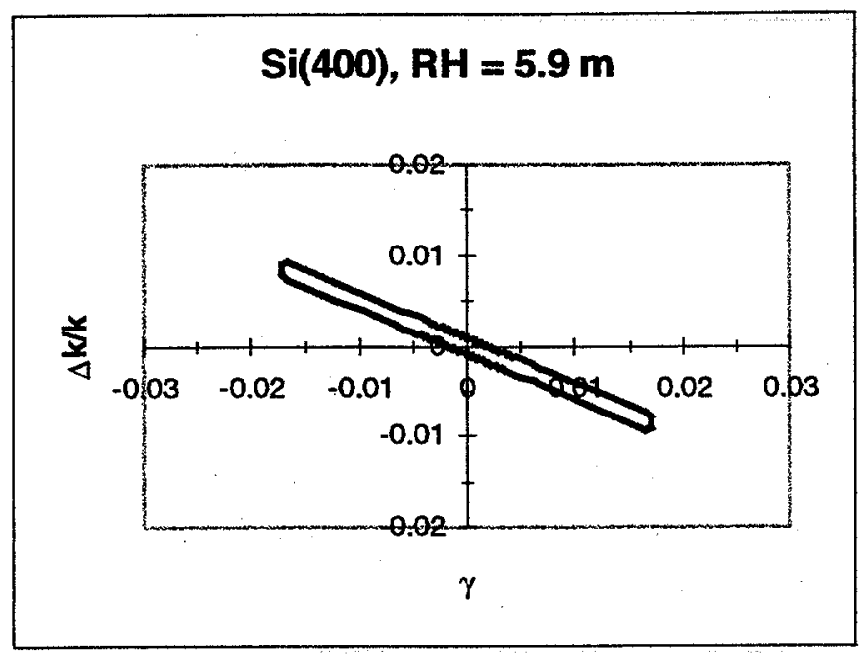

Fig. 2.15

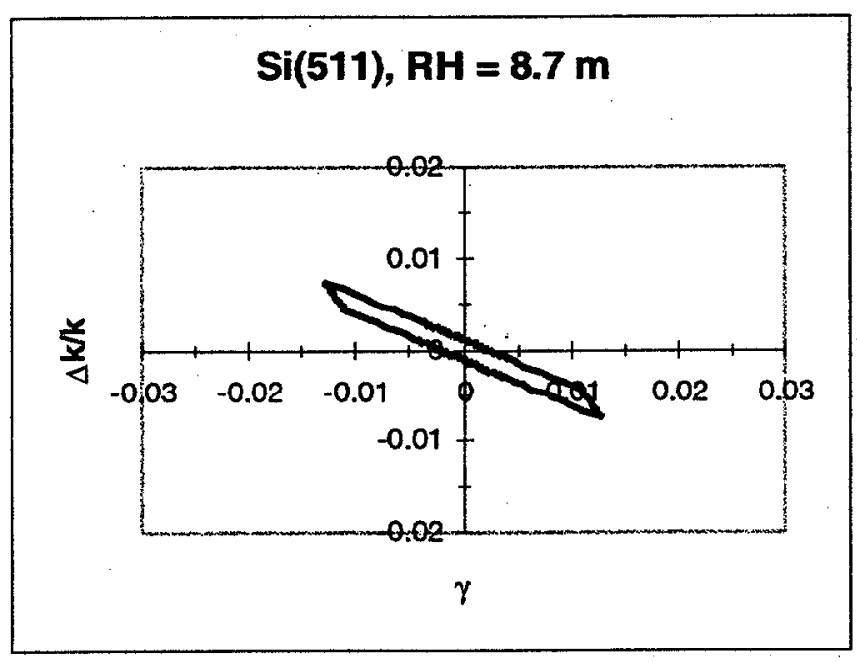

Fig. 2.16 


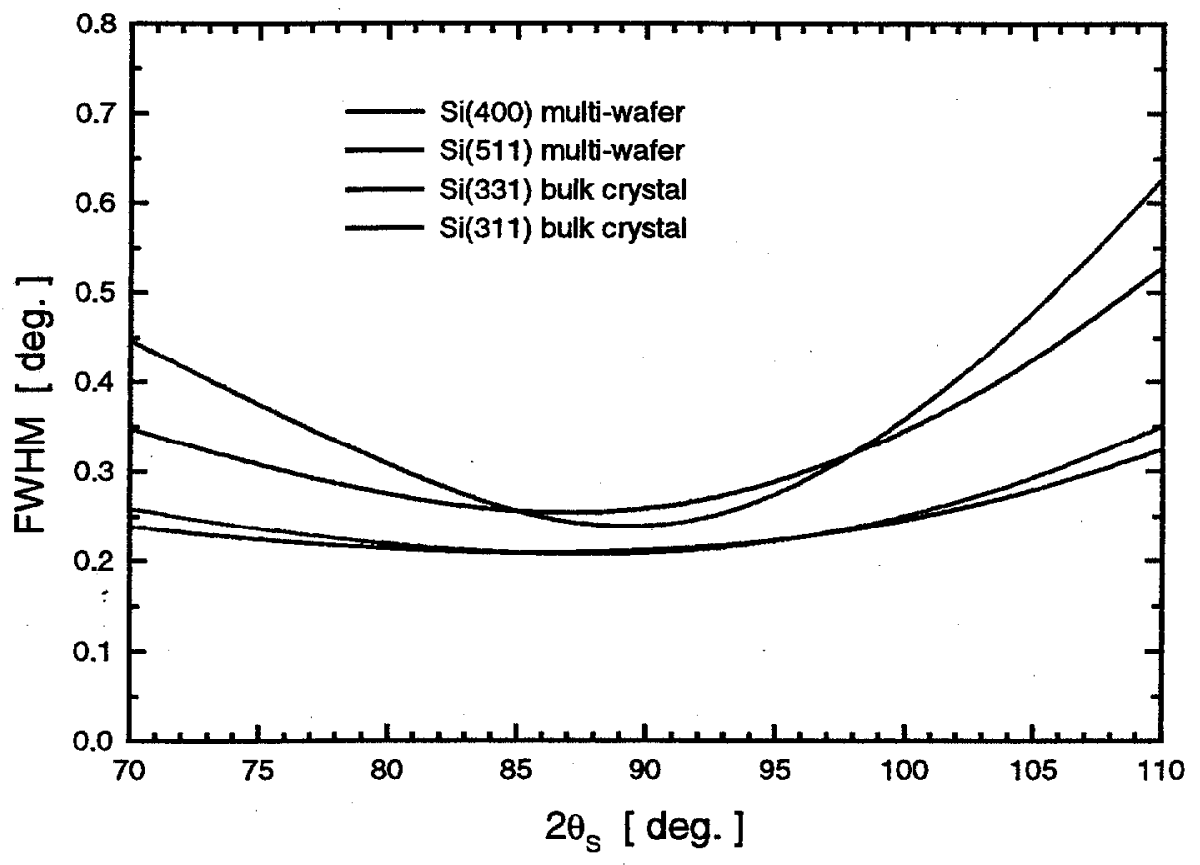

Fig. 2.17 


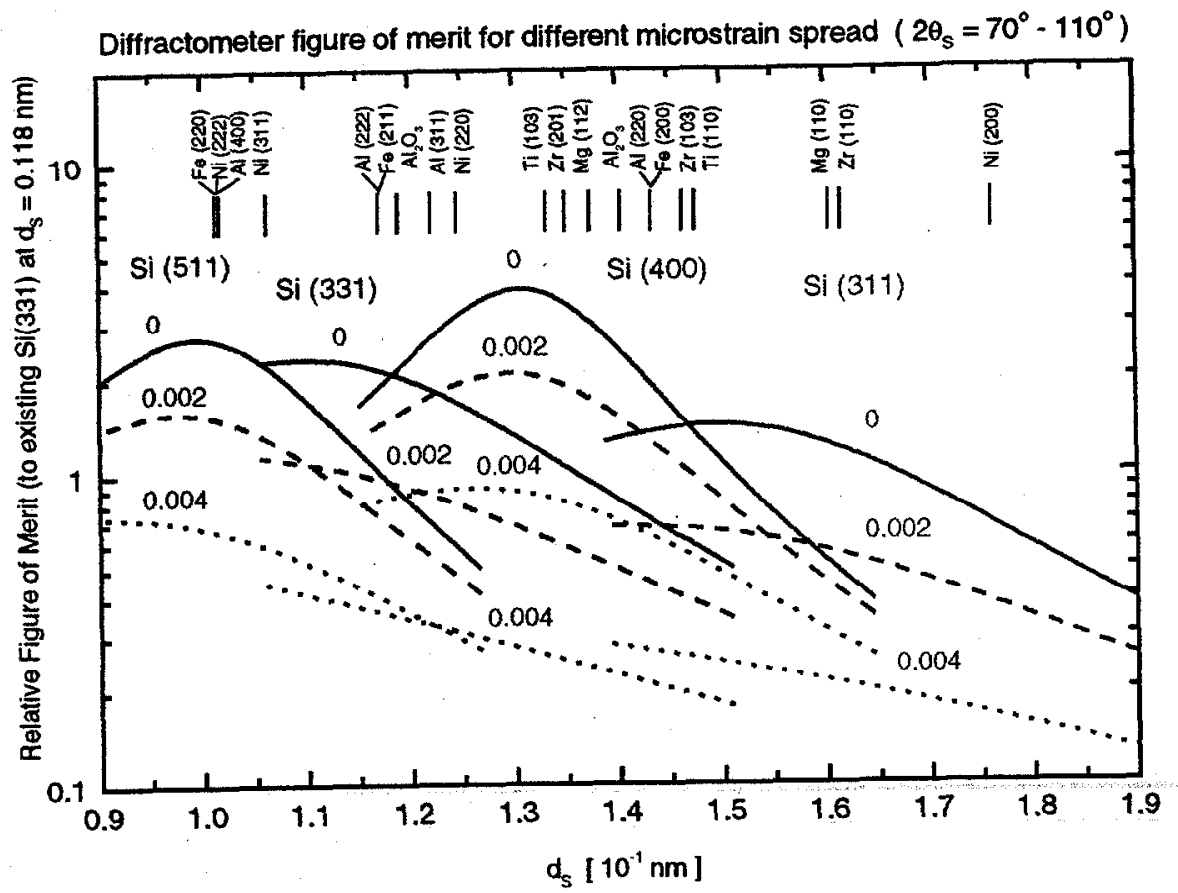

Fig. 2.18 


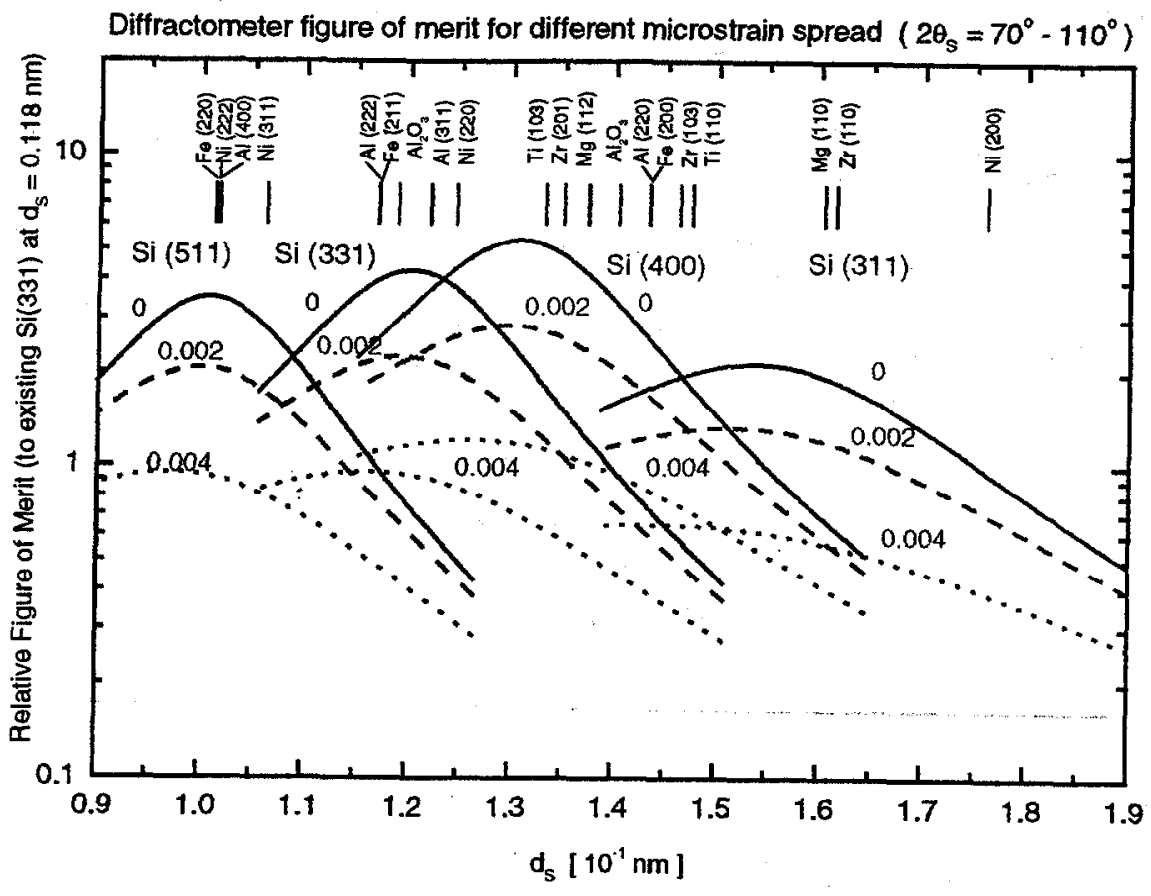

Fig. 2.19 


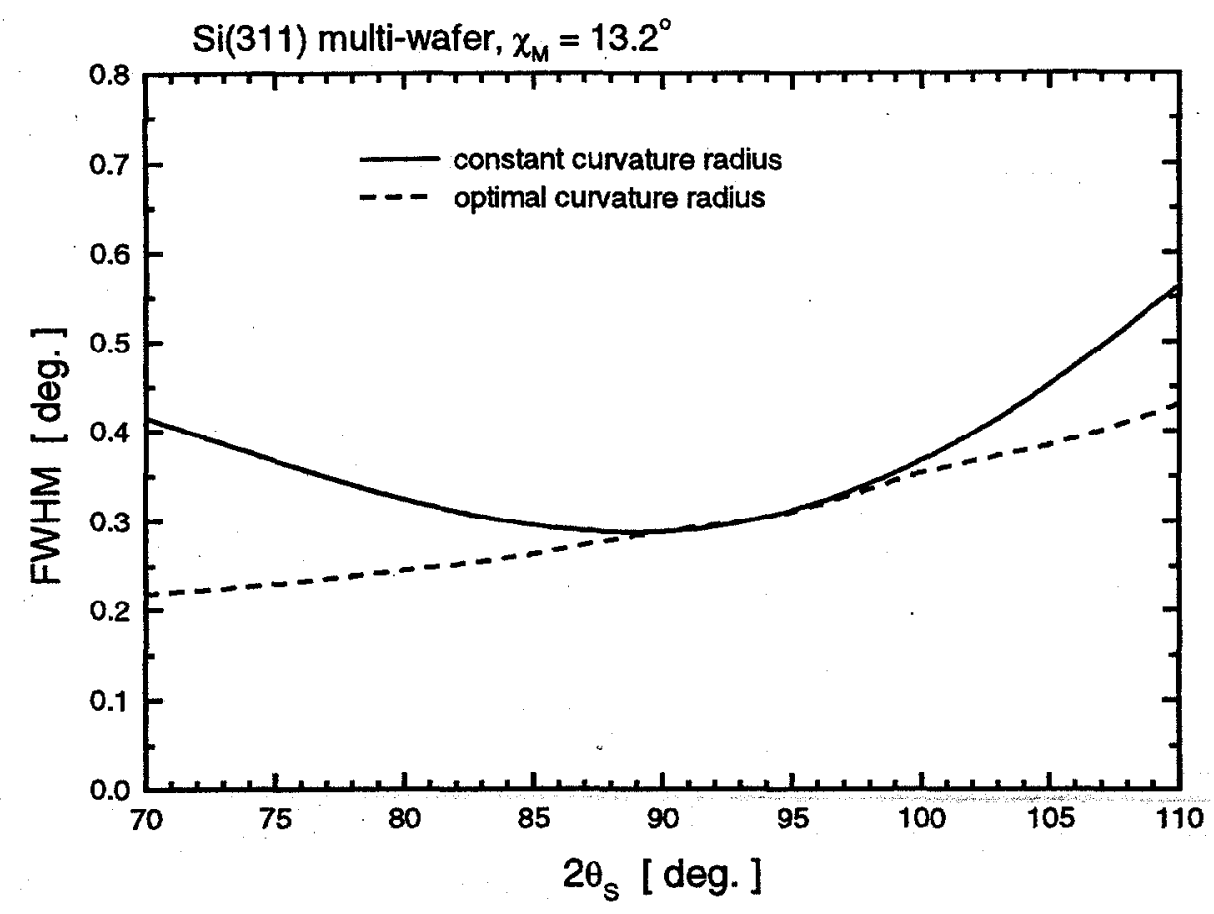

Fig. 2.20

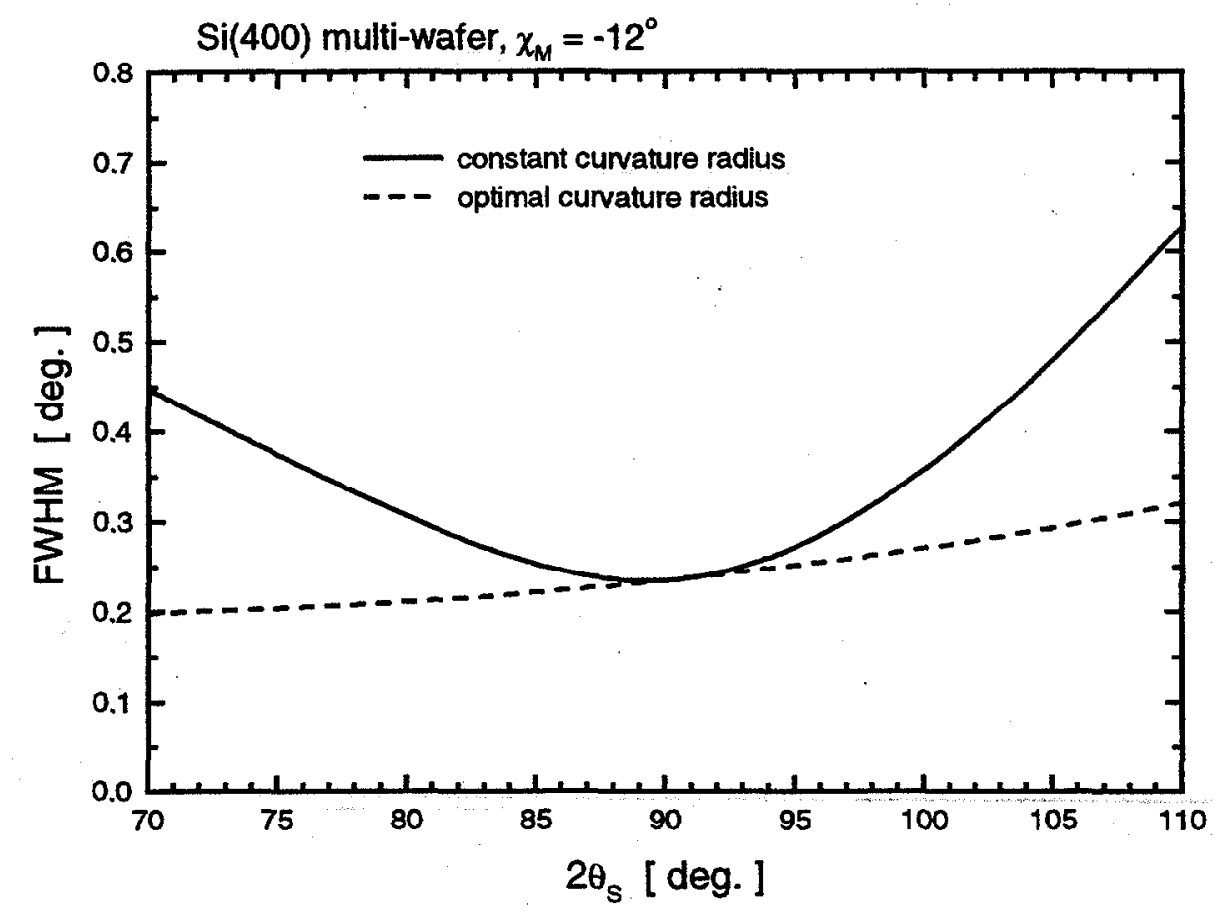

Fig. 2.21 


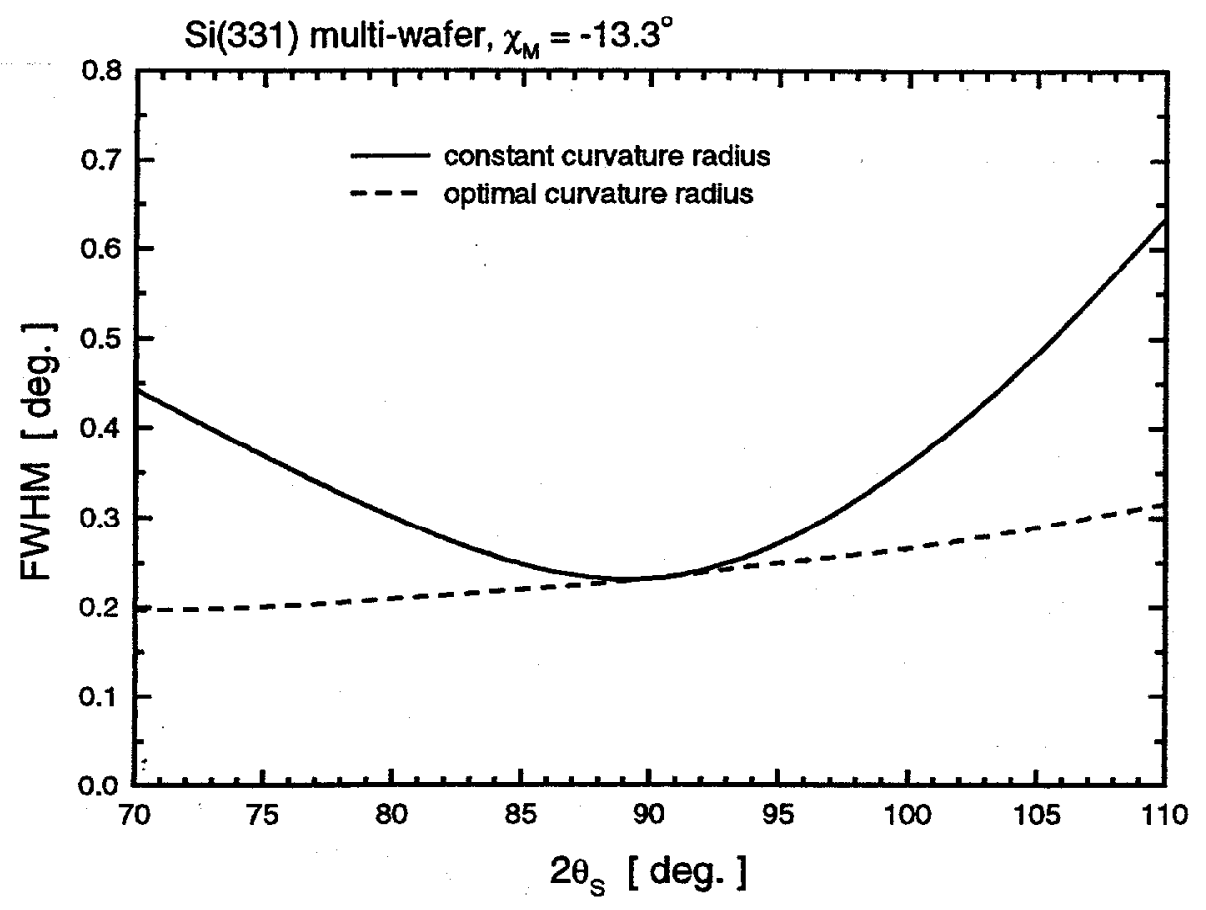

Fig. 2.22

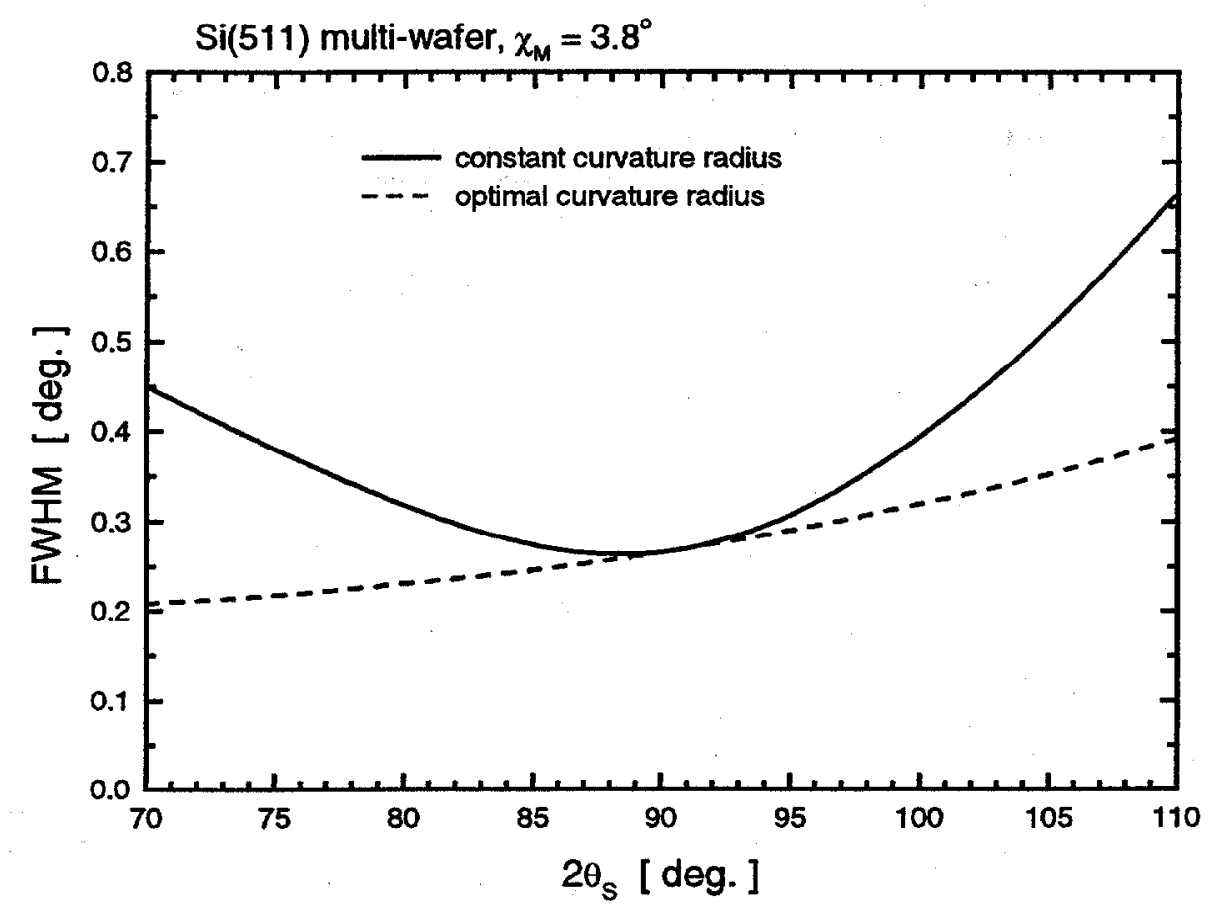

Fig. 2.23 


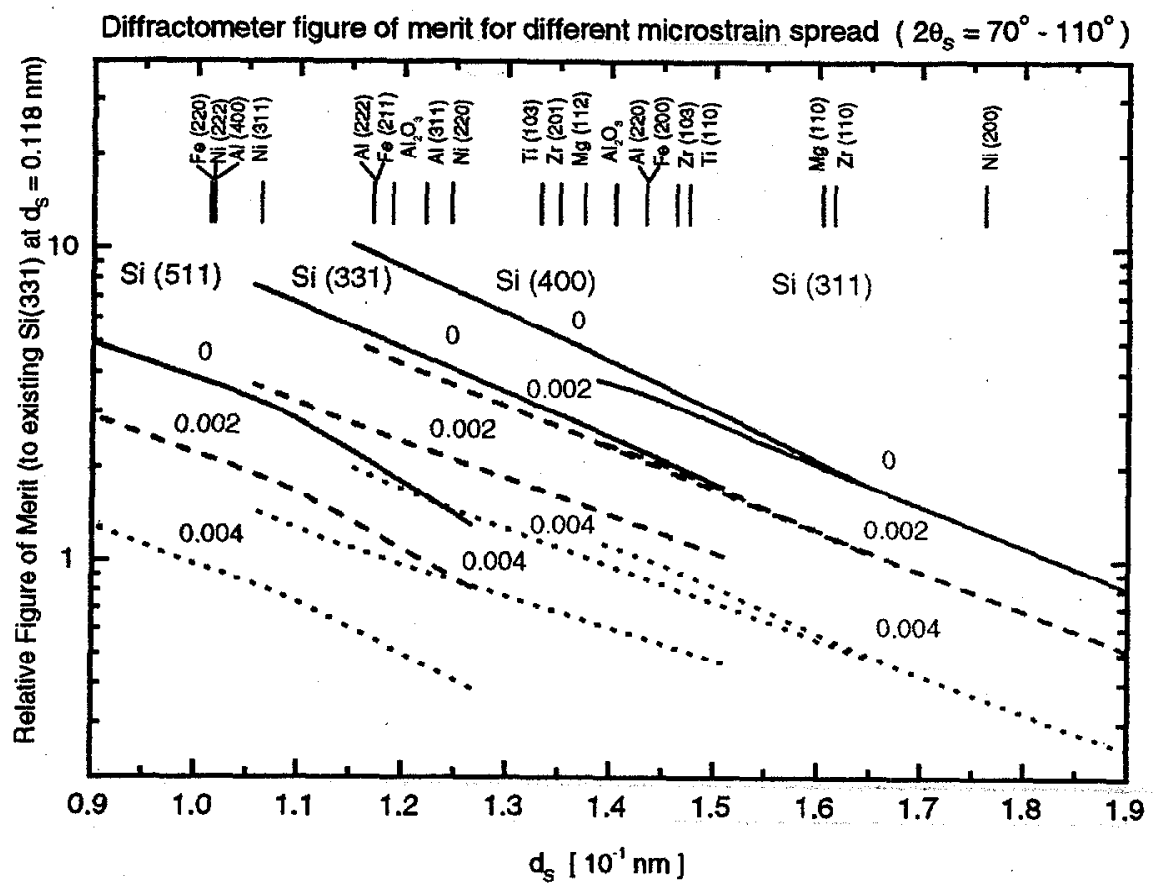

Fig. 2.24 


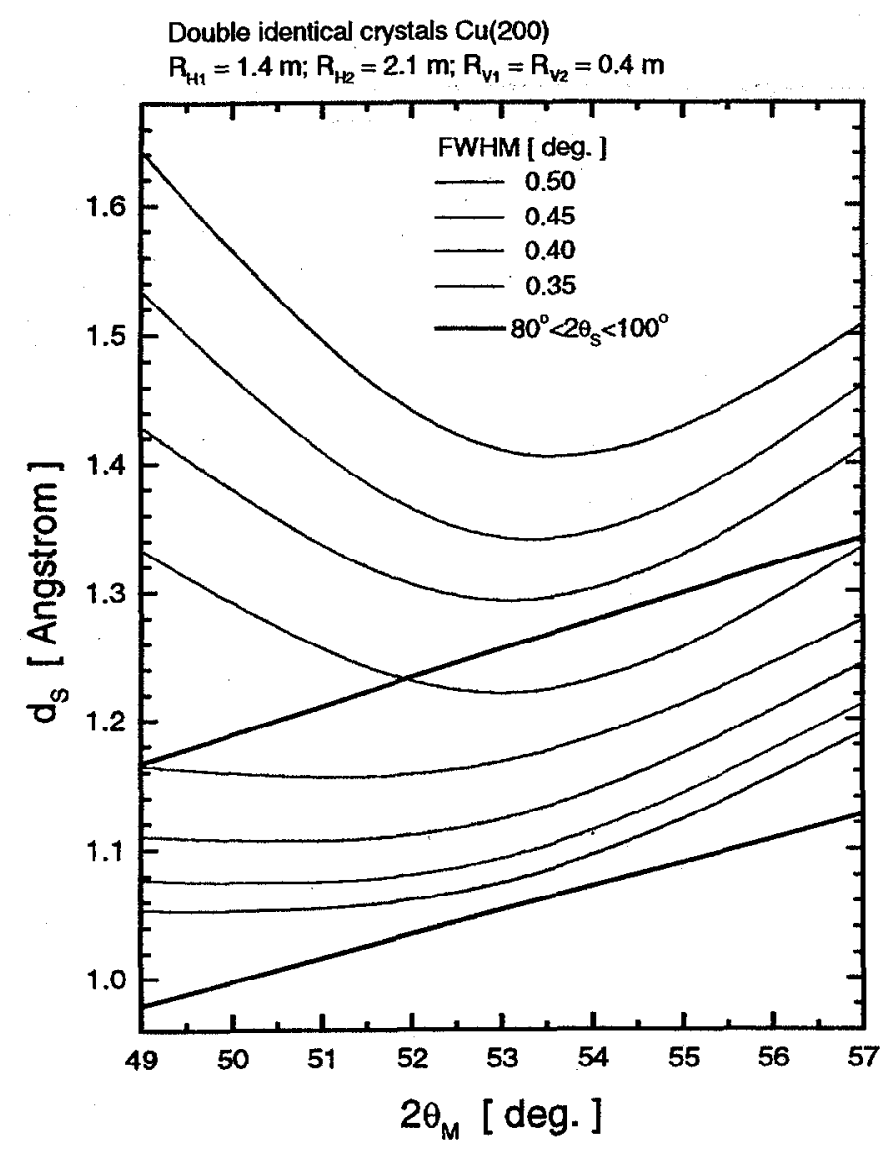

Fig. 3.1 


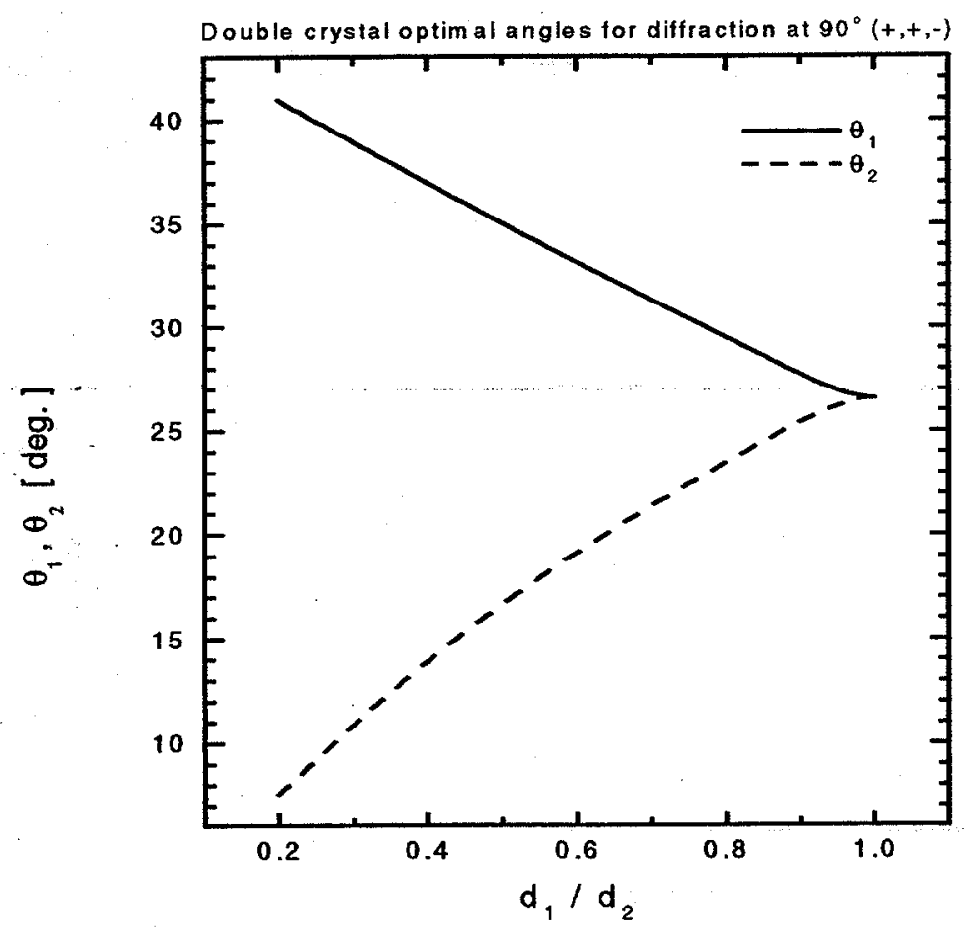

Fig. 3.2

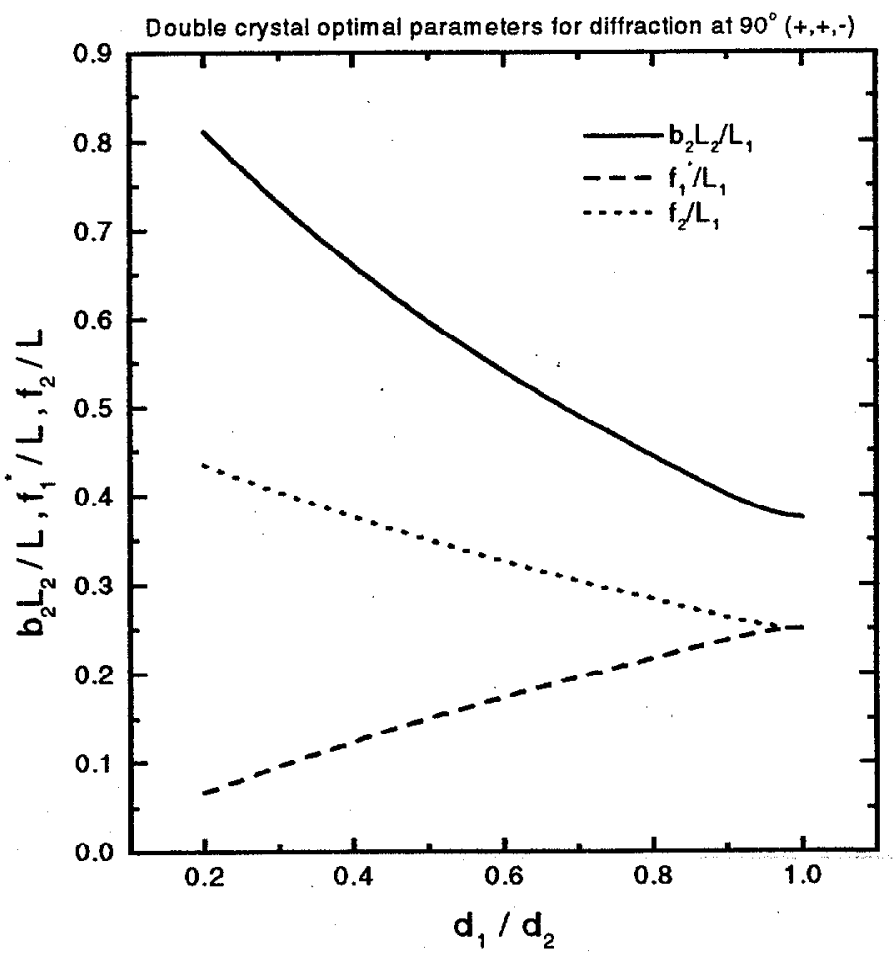

Fig. 3.3 


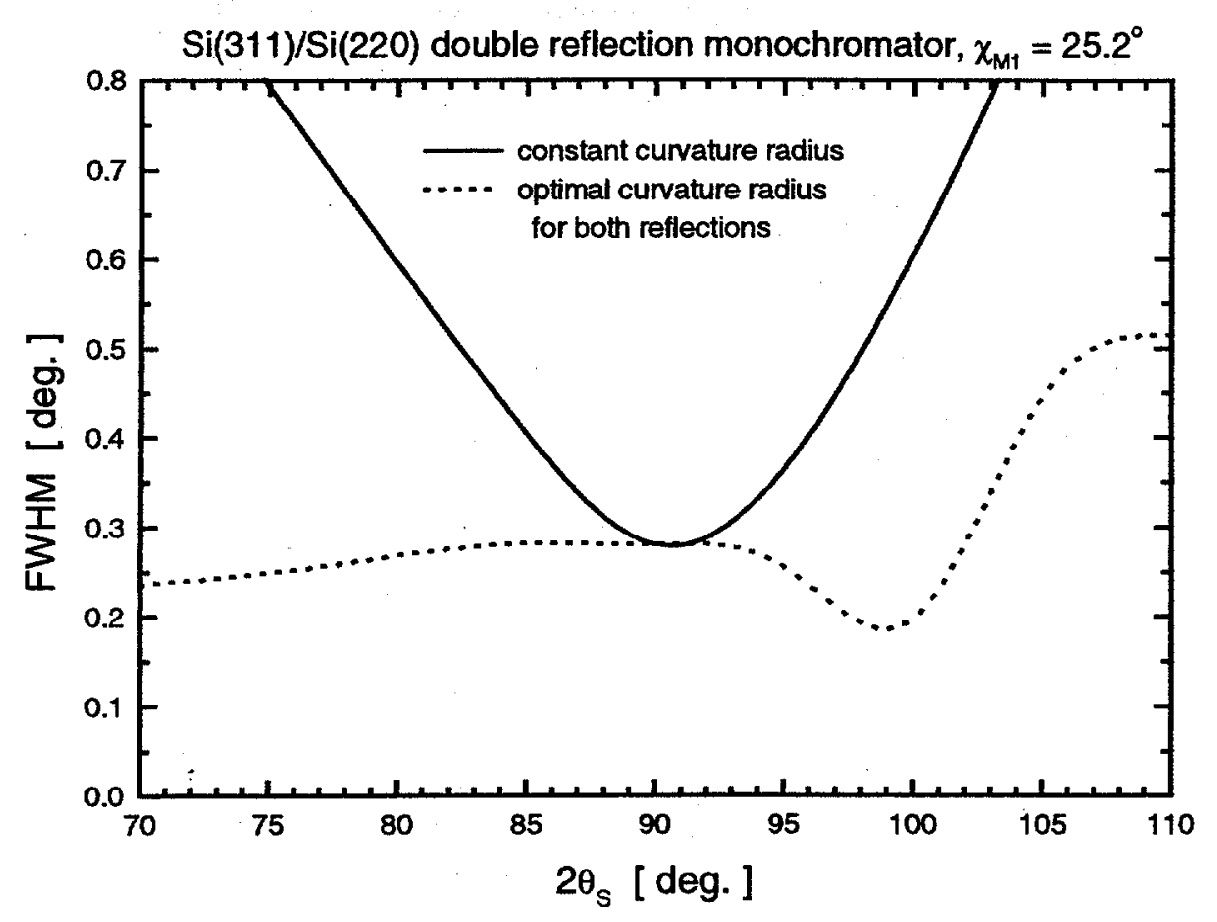

Fig. 3.5

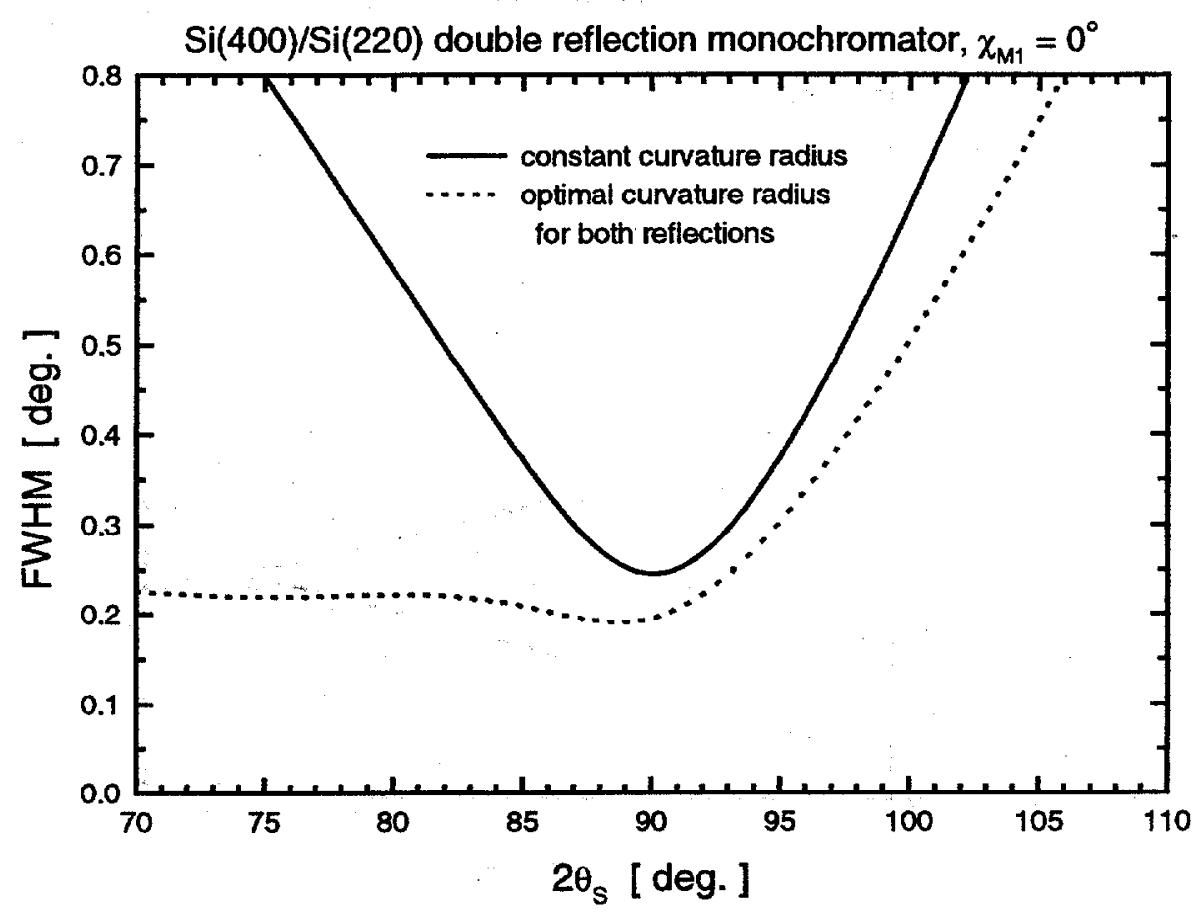

Fig. 3.6 


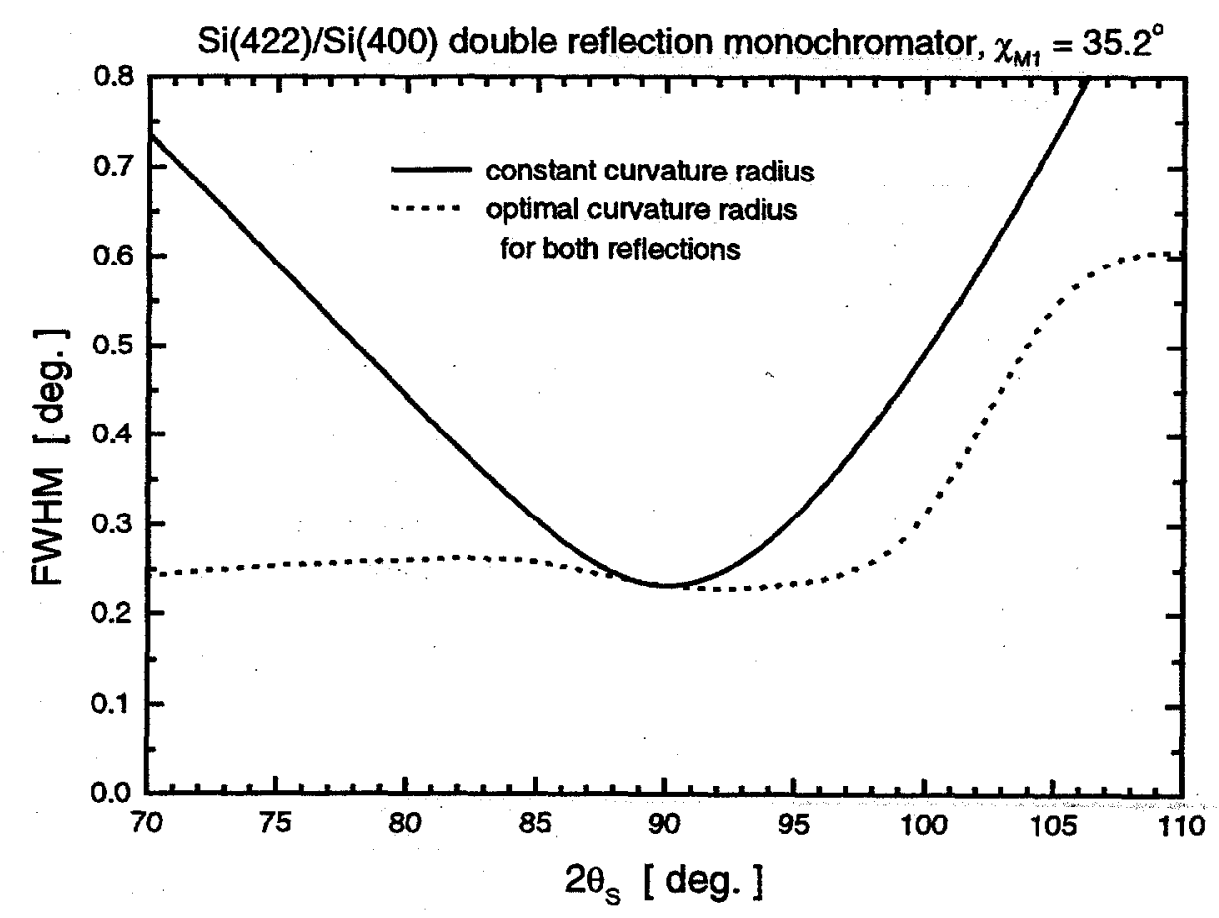

Fig. 3.7

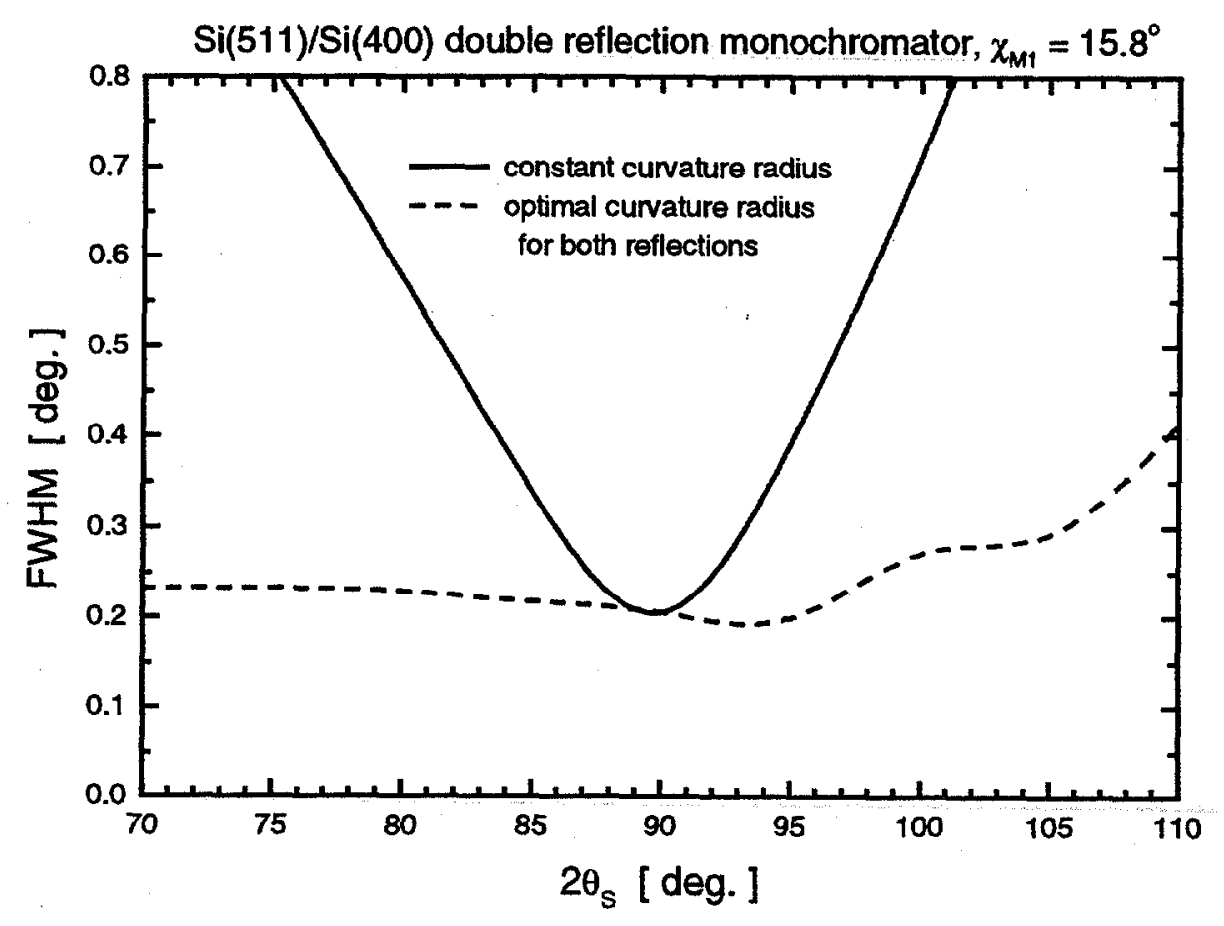

Fig. 3.8 


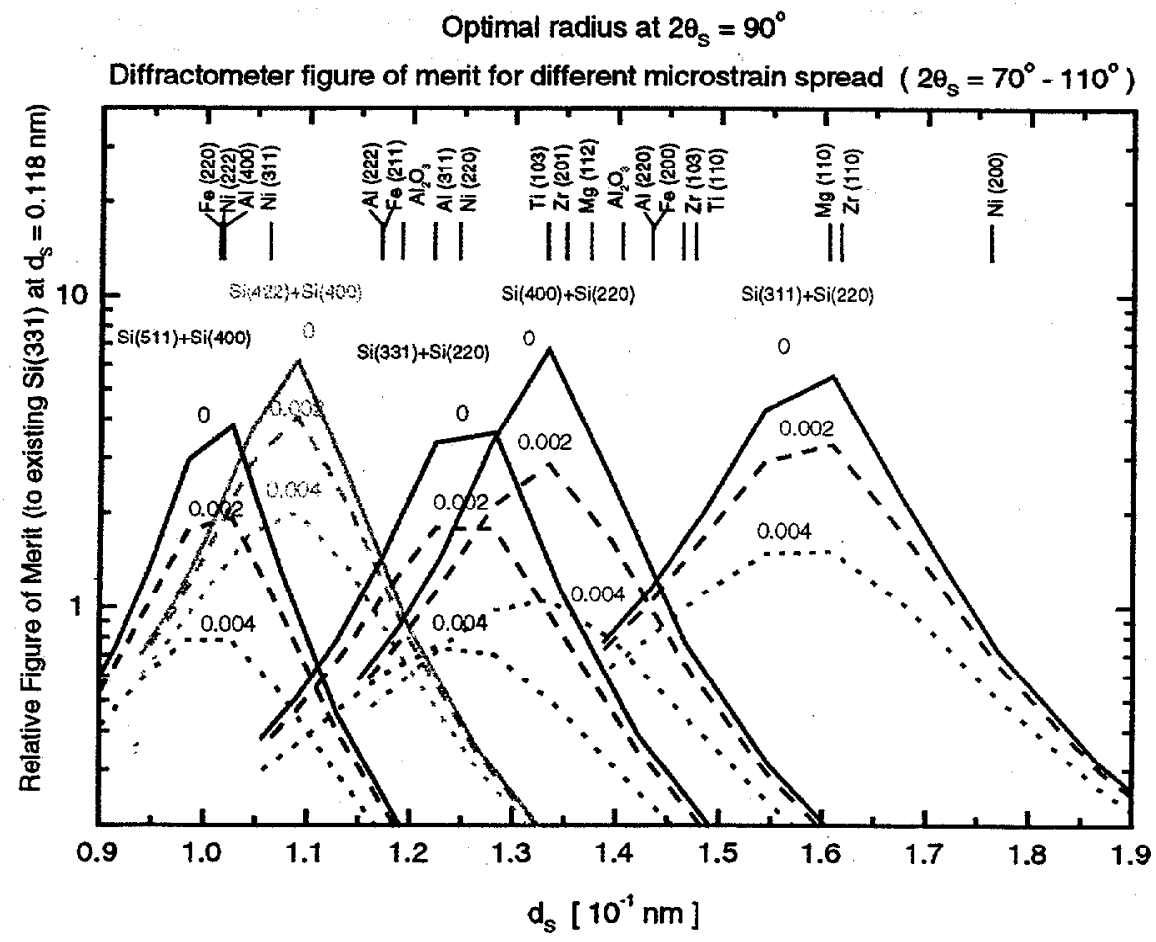

Fig. 3.9 
Optimal radius for each scattering angle

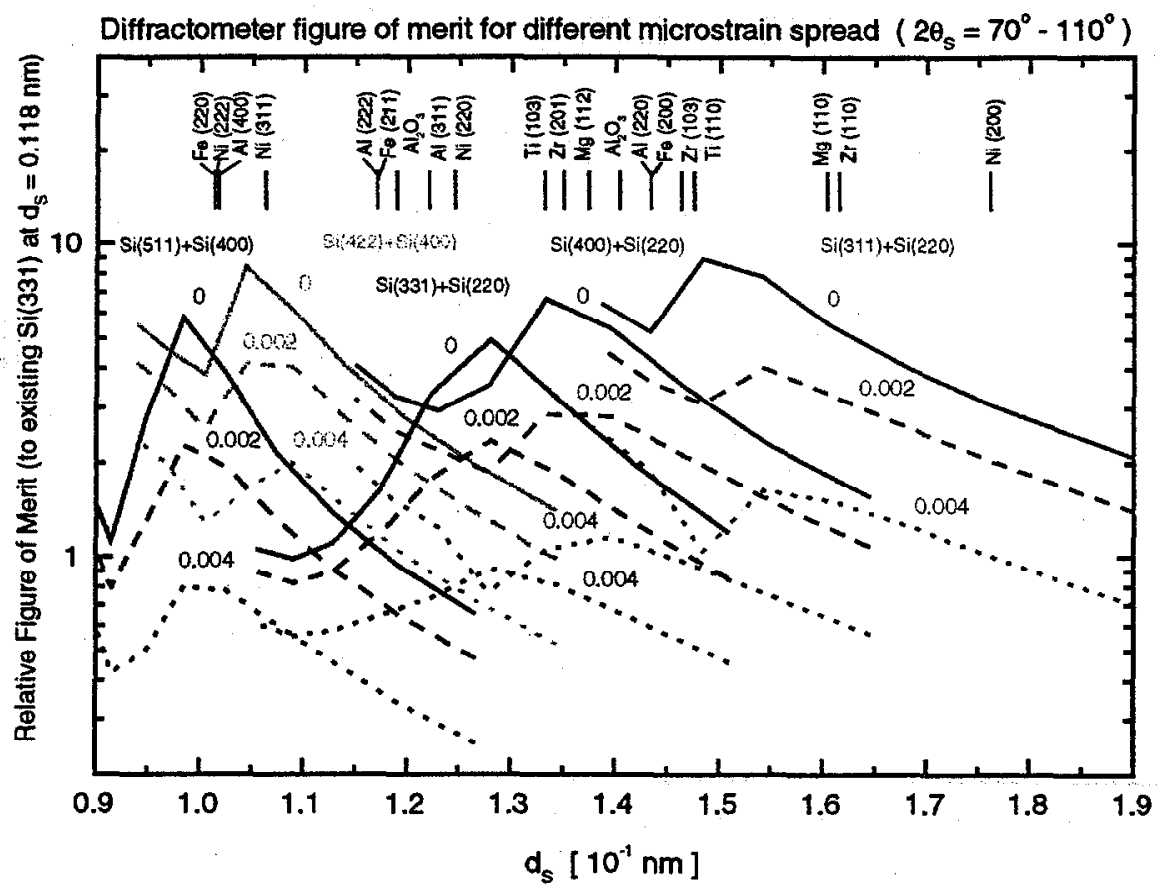

Fig. 3.10 


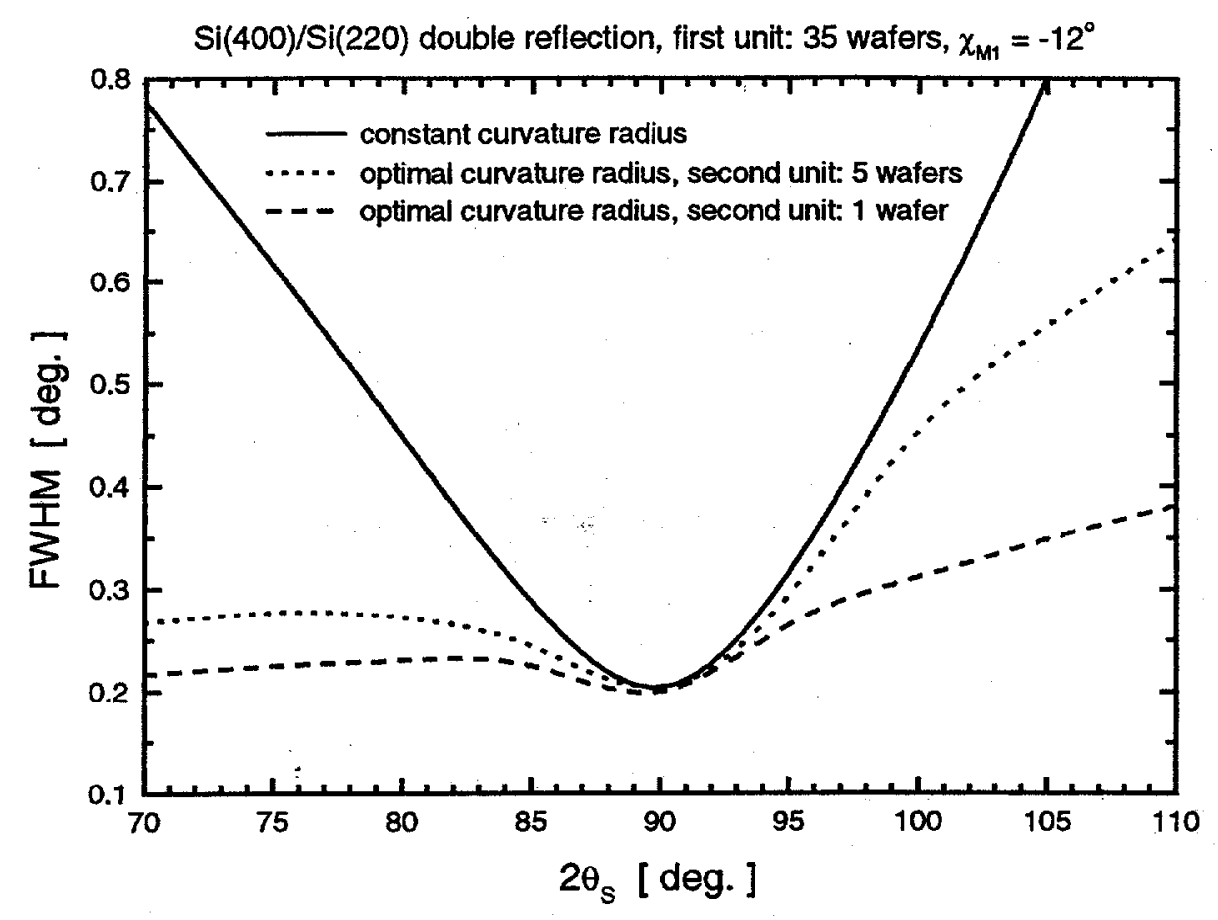

Fig. 3.11

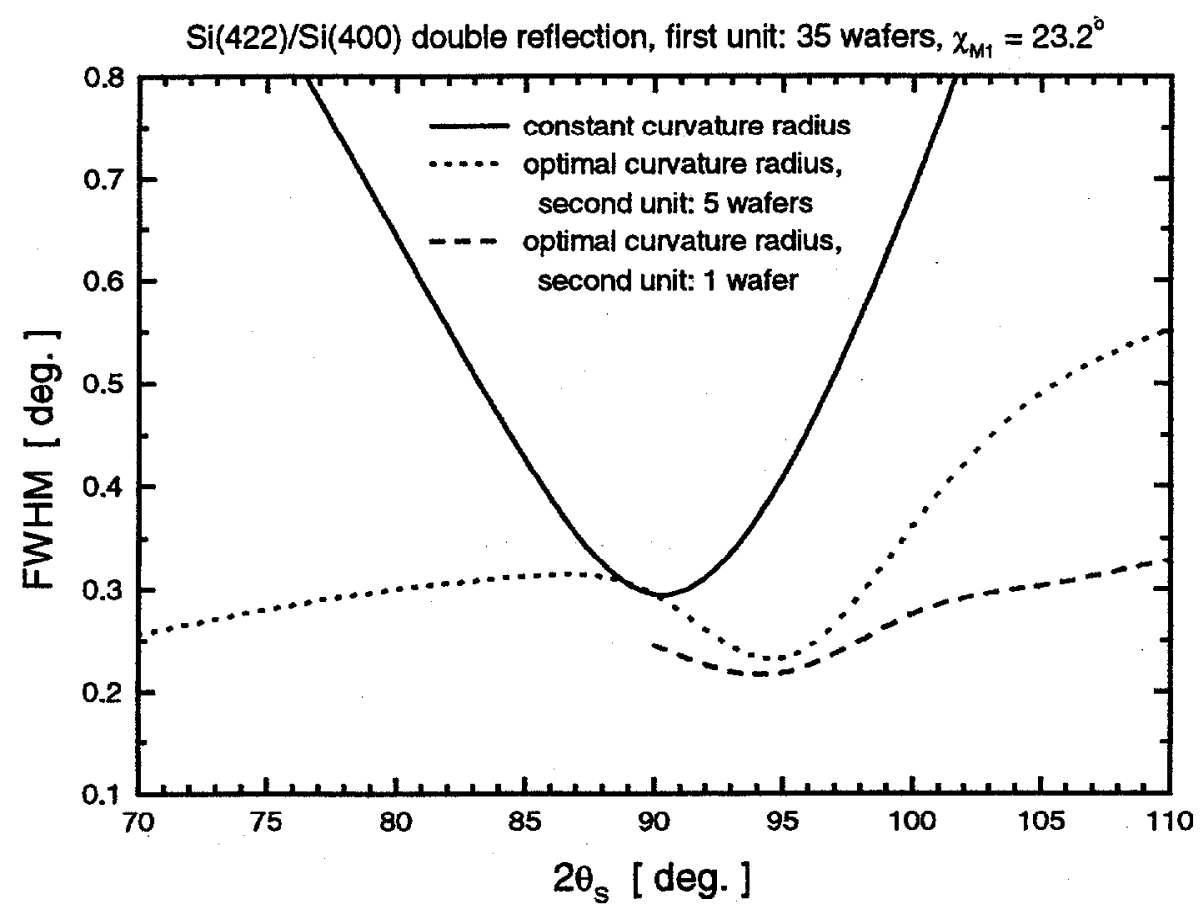

Fig. 3.12 


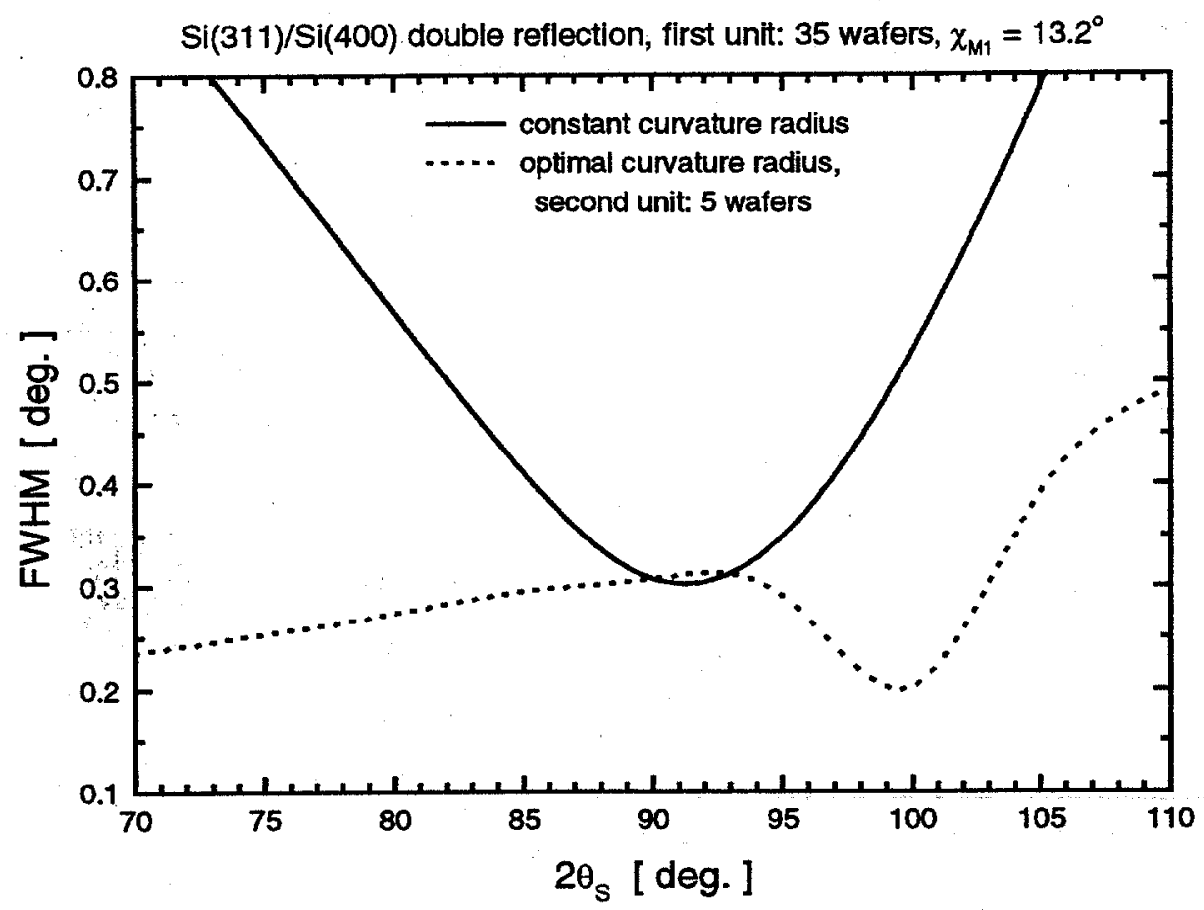

Fig. 3.13

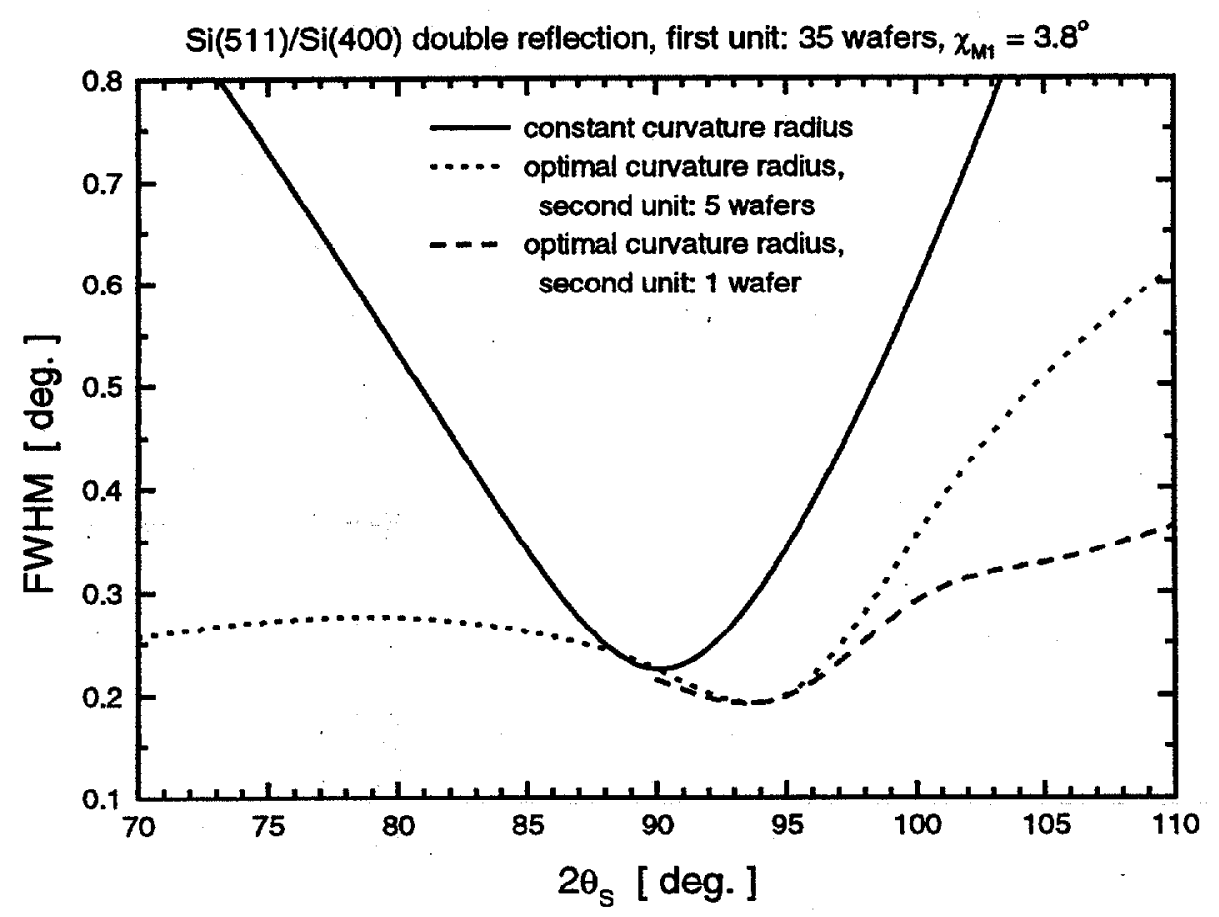

Fig. 3.14 


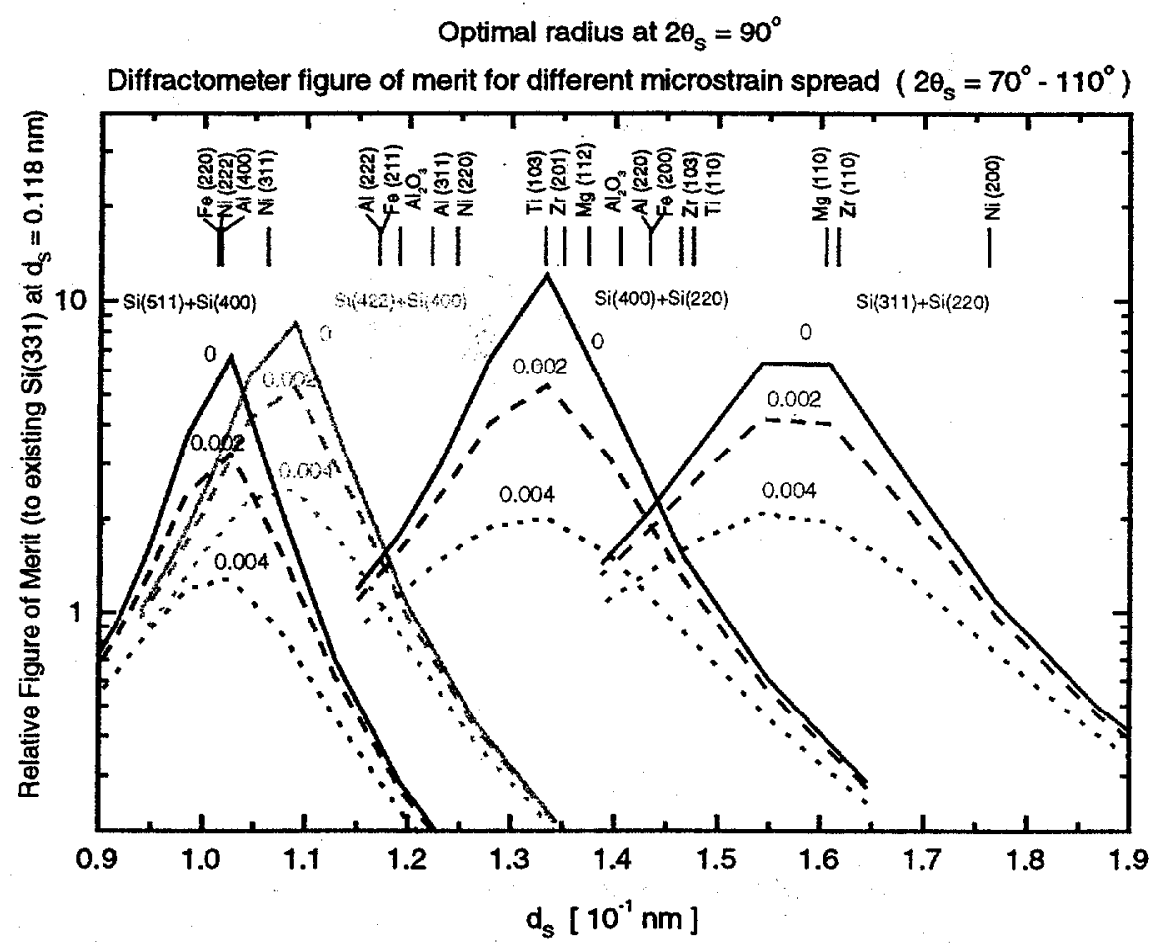

Fig. 3.15 


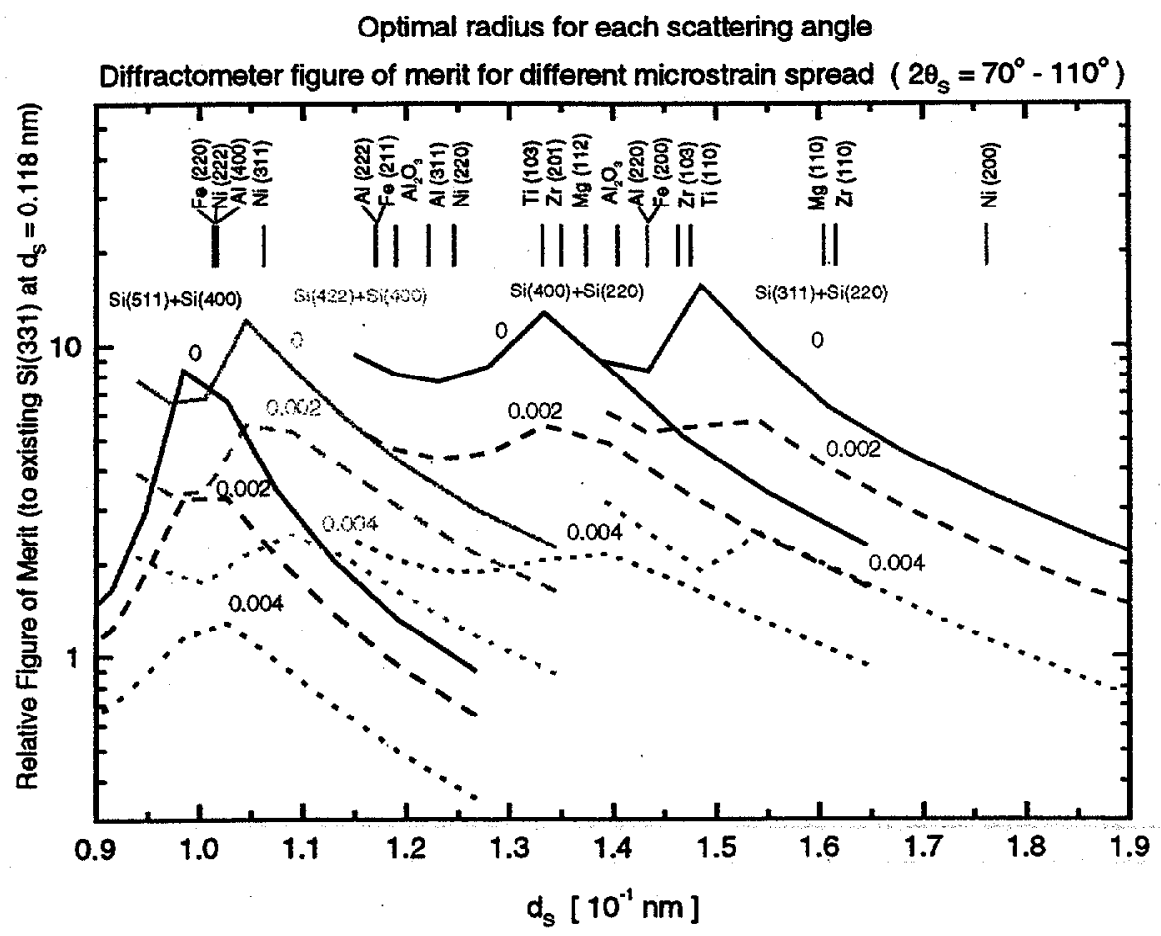

Fig. 3.16 
